\title{
Ligand-Enabled, Copper-Catalyzed Regio- and Stereoselective Synthesis of Trialkylsubstituted Alkenylboronates from Unactivated Internal Alkynes
}

\author{
Taisuke Itoh, Yohei Shimizu and Motomu Kanai* \\ Graduate School of Pharmaceutical Sciences, The University of Tokyo, \\ and JST-ERATO, Kanai Life Science Catalysis Project
}

\section{Supporting Information}

Table of Contents

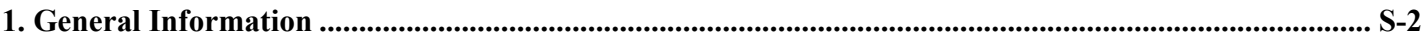

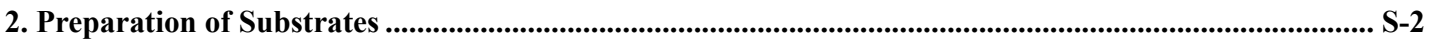

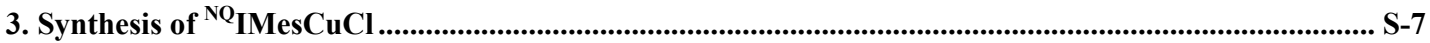

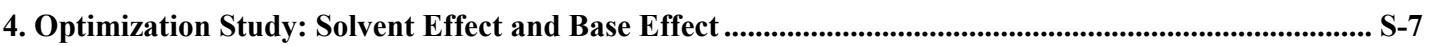

5. Copper-Catalyzed Regioselective Borylalkylation of Dialkylsubstituted Internal Alkynes ..................... S-8

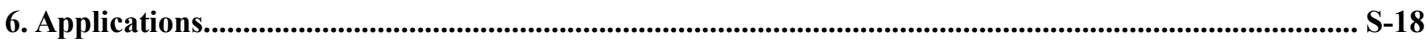

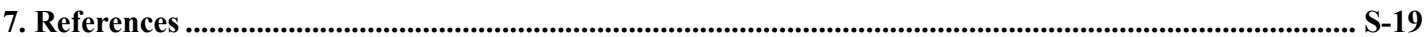

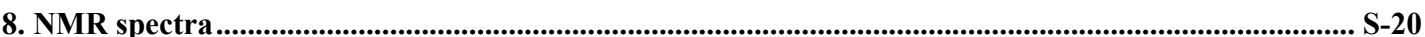




\section{General Information}

NMR spectra were recorded on JEOL ECX500 (500 MHz for ${ }^{1} \mathrm{H}$ NMR and $125 \mathrm{MHz}$ for ${ }^{13} \mathrm{C}$ NMR) spectrometers. Chemical shifts were reported in ppm on the $\delta$ scale relative to TMS $\left(\delta=0.00\right.$ for ${ }^{1} \mathrm{H}$ NMR and $\delta=0.00$ for ${ }^{13} \mathrm{C}$ NMR $)$ as an internal reference. Infrared (IR) spectra were recorded on a JASCO FT/IR 410 Fourier transform infrared spectrophotometer. ESI- and DART-mass spectra were measured on a JEOL JMS-T100LC AccuTOF spectrometer for HRMS. Microwave reactions were performed on Biotage Initiator. Normal phase column chromatographies were performed with Biotage Isolera One and Biotage SNAP Ultra. Gel permeation chromatographies (GPC) were performed with JAI LC-9210 NEXT. Reactions were carried out in dry solvents under an argon atmosphere, unless otherwise stated. Reagents were used as received from commercial sources, unless otherwise stated.

\section{Preparation of Substrates}

2-Octyne (2b), 4-methyl-1-butyne (2c), 3-hexyne (2d) and 1-phenyl-1-hexyne (2m) were purchased from commercial vendors and used after Kugelrohr distillation. 5-Phenyl-1-pentyne (2n) was purchased from a commercial vendor and used after silica gel column chromatography (eluent: hexane). 1-Iodobutane (3a), 1-iodo-3-phenylpropane (3b), (iodomethyl)cyclopropane (3e), iodomethane (3f), benzyl bromide (3g), ethyl bromoacetate (3h), 1-iodo-2-methylpropane (3i), (Bpin $)_{2}, \mathrm{KO} t \mathrm{Bu}$, and dehydrated DMF were purchased from commercial vendors and used without any purification. tert-Butyldimethylsilyl(3-pentynyloxy)silane (2e) was synthesized referring to the reported procedure. ${ }^{1}$

\section{Benzyl 3-pentynyl ether (2a)}

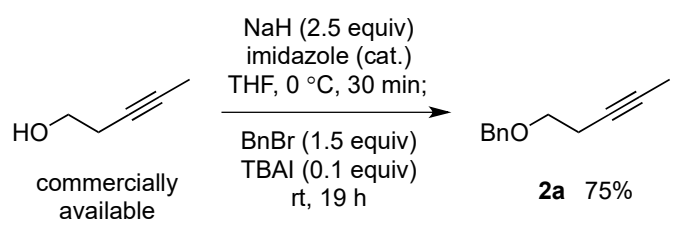

To a dried $300 \mathrm{~mL}$ flask were added $\mathrm{NaH}(3.25 \mathrm{~g}, 81.3 \mathrm{mmol})$ and $\mathrm{THF}(100 \mathrm{~mL})$. The resulting mixture was stirred at $0{ }^{\circ} \mathrm{C}$, before 3-pentyn-1-ol (3.00 mL, $32.5 \mathrm{mmol})$ and imidazole (1 small crystal) were added. The resulting mixture was stirred at $0{ }^{\circ} \mathrm{C}$ for $30 \mathrm{~min}$, before benzyl bromide $(5.80 \mathrm{~mL}, 48.8 \mathrm{mmol})$ and TBAI $(1.20 \mathrm{~g}, 3.25 \mathrm{mmol})$ were added. The resulting mixture was stirred at ambient temperature for $19 \mathrm{~h}$. The reaction was quenched with water, and extracted three times with ether. The combined organic layer was washed with brine, and dried over $\mathrm{Na}_{2} \mathrm{SO}_{4}$. After filtration and evaporation, the residue was purified by silica gel column chromatography (eluent: hexane/AcOEt $=49 / 1 \rightarrow 19 / 1$ ) to give pale yellow oil. After Kugelrohr distillation, pure 2a was obtained as colorless oil (4.24 g, $24.3 \mathrm{mmol}, 75 \%)$. The spectral data were identical with the reported value. ${ }^{2}$ 


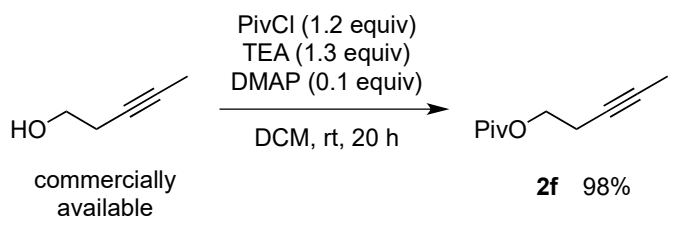

To a dried $200 \mathrm{~mL}$ flask were added 3-pentyn-1-ol (2.00 mL, $21.8 \mathrm{mmol})$, triethylamine (4.00 mL, $28.4 \mathrm{mmol})$, DMAP $(0.270 \mathrm{~g}, 2.18 \mathrm{mmol})$ and DCM $(44 \mathrm{~mL})$. The resulting mixture was stirred at $0{ }^{\circ} \mathrm{C}$, before pivaloyl chloride $(3.20 \mathrm{~mL}, 26.2$ mmol) was added. The resulting mixture was stirred at $0{ }^{\circ} \mathrm{C}$ for $5 \mathrm{~min}$, and then at ambient temperature for $20 \mathrm{~h}$. The reaction mixture was filtrated through a short pad of silica gel (eluted with DCM). After evaporation, the residue was purified by silica gel column chromatography (eluent: hexane/AcOEt $=9 / 1$ ) to give colorless oil. After Kugelrohr distillation, pure $2 \mathbf{f}$ was obtained as colorless oil (3.58 g, $21.3 \mathrm{mmol}, 98 \%)$.

${ }^{1} \mathrm{H} \mathrm{NMR}\left(500 \mathrm{MHz}, \mathrm{CDCl}_{3}\right): \delta 4.11(\mathrm{t}, J=7.0 \mathrm{~Hz}, 2 \mathrm{H}), 2.45$ (qt, $\left.J=2.4 \mathrm{~Hz}, 7.0 \mathrm{~Hz}, 2 \mathrm{H}\right), 1.76(\mathrm{t}, J=2.4 \mathrm{~Hz}, 3 \mathrm{H}), 1.20$ (s, 9H); ${ }^{13} \mathrm{C}$ NMR (125 MHz, $\mathrm{CDCl}_{3}$ ): $\delta$ 178.3, 77.1, 74.7, 62.5, 38.7, 27.1, 19.2, 3.4; IR (neat, $\mathrm{cm}^{-1}$ ): 3444, 2972, 2922, 2873, 1809, 1730, 1481, 1460, 1398, 1366, 1283, 1226, 1154, 1039, 1006, 941, 916, 867, 833, 770, 734; HRMS (ESI): $\mathrm{m} / z$ calcd for $\mathrm{C}_{10} \mathrm{H}_{16} \mathrm{NaO}_{2}^{+}\left[\mathrm{M}+\mathrm{Na}^{+}\right]$: 191.1043. Found: 191.1034.

\section{5-Heptynoic acid}

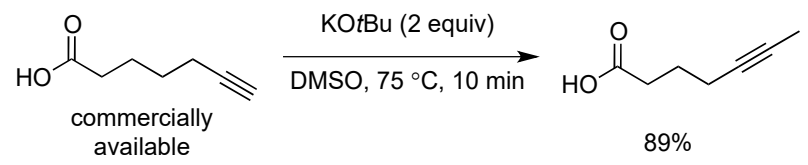

To a dried $50 \mathrm{~mL}$ flask were added 6-heptynoic acid (0.980 g, $7.70 \mathrm{mmol}), \mathrm{KO} t \mathrm{Bu}(1.73 \mathrm{~g}, 15.4 \mathrm{mmol})$ and DMSO (15 mL). The resulting mixture was stirred at $75^{\circ} \mathrm{C}$ for $10 \mathrm{~min}$, and then cooled with ice bath. The reaction was quenched with $1 \mathrm{M}$ aq. $\mathrm{HCl}(20 \mathrm{~mL})$, and extracted three times with ether. The combined organic layer was washed successively with water and brine, and dried over $\mathrm{Na}_{2} \mathrm{SO}_{4}$. After filtration and evaporation, the residue was purified by silica gel column chromatography (eluent: hexane/AcOEt $=4 / 1)$ to give 5-heptynoic acid as white solids $(0.860 \mathrm{~g}, 6.82 \mathrm{mmol}, 89 \%)$. The spectral data were identical with the reported value. ${ }^{3}$

\section{Methyl 5-heptynoate (2g)}

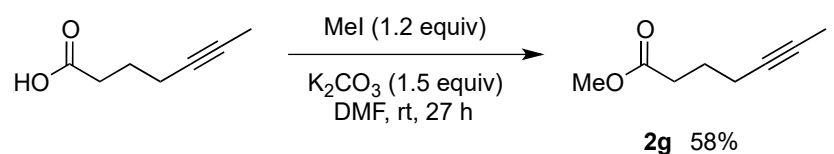

To a dried $30 \mathrm{~mL}$ flask were added 5-heptynoic acid (0.38 g, $3.0 \mathrm{mmol}), \mathrm{K}_{2} \mathrm{CO}_{3}(0.62 \mathrm{~g}, 4.5 \mathrm{mmol})$ and DMF (6.0 mL). The resulting mixture was stirred at $0{ }^{\circ} \mathrm{C}$, before iodomethane $(0.22 \mathrm{~mL}, 3.6 \mathrm{mmol})$ was added. The resulting mixture was stirred at ambient temperature for $27 \mathrm{~h}$. The reaction was quenched with water, and extracted three times with ether. The combined organic layer was dried over $\mathrm{Na}_{2} \mathrm{SO}_{4}$. After filtration and evaporation, the residue was purified by silica gel column 
chromatography (eluent: hexane/AcOEt $=19 / 1)$ to give $\mathbf{2 g}$ as colorless oil $(0.24 \mathrm{~g}, 1.7 \mathrm{mmol}, 58 \%)$. The spectral data were identical with the reported value. ${ }^{4}$

\section{$N, N$-diethyl-5-heptynamide $(2 \mathrm{~h})$}

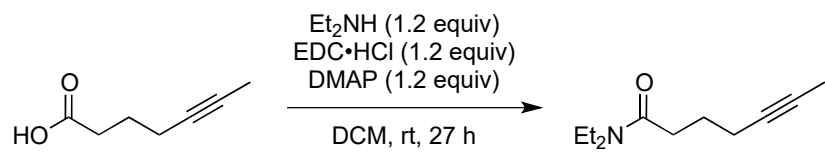

2h $79 \%$

To a dried $50 \mathrm{~mL}$ flask were added 5-heptynoic acid $(0.38 \mathrm{~g}, 3.0 \mathrm{mmol})$, EDC $\cdot \mathrm{HCl}(0.69 \mathrm{~g}, 3.6 \mathrm{mmol})$, DMAP $(0.44 \mathrm{~g}, 3.6$ $\mathrm{mmol})$ and DCM $(15 \mathrm{~mL})$. To the resulting mixture was added diethylamine $(0.37 \mathrm{~mL}, 3.6 \mathrm{mmol})$. The resulting mixture was stirred at ambient temperature for $27 \mathrm{~h}$. The reaction was quenched with $10 \%$ aq. citric acid, and extracted three times with DCM. The combined organic layer was dried over $\mathrm{Na}_{2} \mathrm{SO}_{4}$. After filtration and evaporation, the residue was purified by silica gel column chromatography (eluent: hexane/AcOEt $=9 / 1 \rightarrow 4 / 1)$ to give $\mathbf{2 h}$ as colorless oil $(0.43 \mathrm{~g}, 2.4 \mathrm{mmol}, 79 \%)$.

${ }^{1} \mathrm{H}$ NMR (500 MHz, $\left.\mathrm{CDCl}_{3}\right): \delta 3.37$ (q, $\left.J=7.1 \mathrm{~Hz}, 2 \mathrm{H}\right), 3.33(\mathrm{q}, J=7.1 \mathrm{~Hz}, 2 \mathrm{H}), 2.42(\mathrm{t}, J=7.4 \mathrm{~Hz}, 2 \mathrm{H}), 2.21$ (qt, $J=2.6$ $\mathrm{Hz}, 6.8 \mathrm{~Hz}, 2 \mathrm{H}), 1.85-1.79(\mathrm{~m}, 2 \mathrm{H}), 1.77$ (t, $J=2.6 \mathrm{~Hz}, 3 \mathrm{H}), 1.18(\mathrm{t}, J=7.1 \mathrm{~Hz}, 3 \mathrm{H}), 1.11(\mathrm{t}, J=7.1 \mathrm{~Hz}, 3 \mathrm{H}) ;{ }^{13} \mathrm{C} \mathrm{NMR}(125$ $\mathrm{MHz}, \mathrm{CDCl}_{3}$ ): $\delta 171.6,78.6,76.1,41.9,40.0,31.7,24.5,18.3,14.3,13.1,3.4$; IR (neat, $\mathrm{cm}^{-1}$ ): 3450, 2976, 2935, 1631, 1457,

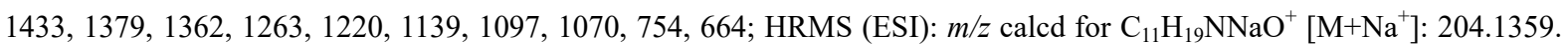
Found: 204.1352.

\section{Benzyl 2-butynyl ether (2i)}

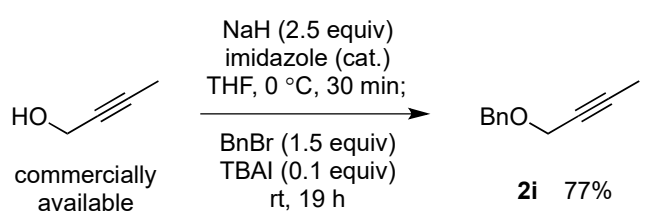

To a dried $200 \mathrm{~mL}$ flask were added $\mathrm{NaH}(2.68 \mathrm{~g}, 67.0 \mathrm{mmol})$ and $\mathrm{THF}(100 \mathrm{~mL})$. The resulting mixture was stirred at $0{ }^{\circ} \mathrm{C}$, before 2-butyn-1-ol (2.00 mL, $26.8 \mathrm{mmol})$ and imidazole (1 small crystal) were added. The resulting mixture was stirred at $0{ }^{\circ} \mathrm{C}$ for $30 \mathrm{~min}$, before benzyl bromide $(4.77 \mathrm{~mL}, 40.2 \mathrm{mmol})$ and TBAI $(0.990 \mathrm{~g}, 2.68 \mathrm{mmol})$ were added. The resulting mixture was stirred at ambient temperature for $19 \mathrm{~h}$. The reaction was quenched with water, and extracted three times with ether. The combined organic layer was washed with brine, and dried over $\mathrm{Na}_{2} \mathrm{SO}_{4}$. After filtration and evaporation, the residue was purified by silica gel column chromatography (eluent: hexane/AcOEt $=49 / 1 \rightarrow 19 / 1$ ) to give pale yellow oil. After Kugelrohr distillation, pure $\mathbf{2 i}$ was obtained as colorless oil (3.30 g, $20.6 \mathrm{mmol}, 77 \%)$. The spectral data were identical with the reported value. ${ }^{5}$

\section{Benzyl 2-pentynyl ether (2j)}




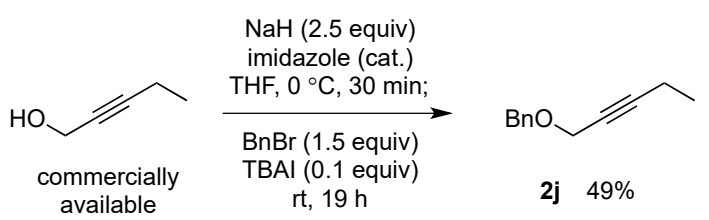

To a dried $200 \mathrm{~mL}$ flask were added $\mathrm{NaH}(2.16 \mathrm{~g}, 54.0 \mathrm{mmol})$ and $\mathrm{THF}(70 \mathrm{~mL})$. The resulting mixture was stirred at $0{ }^{\circ} \mathrm{C}$, before 2-pentyn-1-ol $(2.00 \mathrm{~mL}, 21.6 \mathrm{mmol})$ and imidazole (1 small crystal) were added. The resulting mixture was stirred at $0{ }^{\circ} \mathrm{C}$ for $30 \mathrm{~min}$, before benzyl bromide $(3.85 \mathrm{~mL}, 32.4 \mathrm{mmol})$ and TBAI $(0.800 \mathrm{~g}, 2.16 \mathrm{mmol})$ were added. The resulting mixture was stirred at ambient temperature for $19 \mathrm{~h}$. The reaction was quenched with water, and extracted three times with ether. The combined organic layer was washed with brine, and dried over $\mathrm{Na}_{2} \mathrm{SO}_{4}$. After filtration and evaporation, the residue was purified by silica gel column chromatography (eluent: hexane/AcOEt $=49 / 1 \rightarrow 19 / 1$ ) to give pale yellow oil. After Kugelrohr distillation, pure $\mathbf{2} \mathbf{j}$ was obtained as colorless oil (1.85 g, $10.6 \mathrm{mmol}, 49 \%)$.

${ }^{1} \mathrm{H}$ NMR $\left(500 \mathrm{MHz}, \mathrm{CDCl}_{3}\right): \delta$ 7.36-7.32 (m, 4H), 7.30-7.28 (m, 1H), $4.58(\mathrm{~s}, 2 \mathrm{H}), 4.15(\mathrm{t}, J=2.1 \mathrm{~Hz}, 2 \mathrm{H}), 2.25$ (qt, $J=2.1$ $\mathrm{Hz}, 7.5 \mathrm{~Hz}, 2 \mathrm{H}), 1.16(\mathrm{t}, J=7.5 \mathrm{~Hz}, 3 \mathrm{H}) ;{ }^{13} \mathrm{C} \mathrm{NMR}\left(125 \mathrm{MHz}, \mathrm{CDCl}_{3}\right): \delta 137.6,128.4,128.0,127.7,88.5,75.1,71.4,57.7$, 13.8, 12.4; IR (neat, $\mathrm{cm}^{-1}$ ): 3087, 3064, 3030, 2976, 2937, 2851, 2284, 2225, 1952, 1871, 1810, 1722, 1604, 1586, 1496, $1454,1406,1385,1354,1319,1262,1206,1151,1134,1071,1026,955,936,904,736,698$; HRMS (ESI): $\mathrm{m} / z$ calcd for $\mathrm{C}_{12} \mathrm{H}_{14} \mathrm{NaO}^{+}\left[\mathrm{M}+\mathrm{Na}^{+}\right]:$197.0937. Found: 197.0935 .

\section{Benzyl 3-hexynyl ether $(2 \mathrm{k})$}

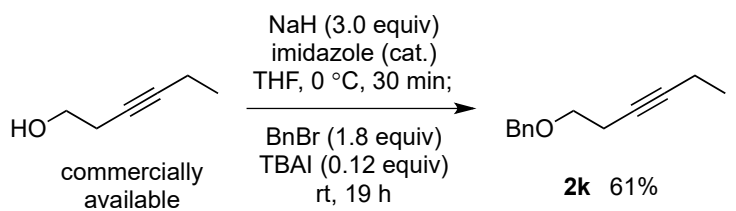

To a dried $200 \mathrm{~mL}$ flask were added $\mathrm{NaH}(2.16 \mathrm{~g}, 54.0 \mathrm{mmol})$ and $\mathrm{THF}(70 \mathrm{~mL})$. The resulting mixture was stirred at $0{ }^{\circ} \mathrm{C}$, before 3-hexyn-1-ol $(2.00 \mathrm{~mL}, 18.2 \mathrm{mmol})$ and imidazole (1 small crystal) were added. The resulting mixture was stirred at $0{ }^{\circ} \mathrm{C}$ for $30 \mathrm{~min}$, before benzyl bromide $(3.85 \mathrm{~mL}, 32.4 \mathrm{mmol})$ and TBAI $(0.800 \mathrm{~g}, 2.16 \mathrm{mmol})$ were added. The resulting mixture was stirred at ambient temperature for $19 \mathrm{~h}$. The reaction was quenched with water, and extracted three times with ether. The combined organic layer was washed with brine, and dried over $\mathrm{Na}_{2} \mathrm{SO}_{4}$. After filtration and evaporation, the residue was purified by silica gel column chromatography (eluent: hexane/AcOEt $=49 / 1 \rightarrow 19 / 1$ ) to give yellow oil. After Kugelrohr distillation, pure $\mathbf{2 k}$ was obtained as colorless oil $(2.08 \mathrm{~g}, 11.0 \mathrm{mmol}, 61 \%)$. The spectral data were identical with the reported value. ${ }^{2}$ 
$N, N$-dibenzyl-2-heptynylamine (2I)

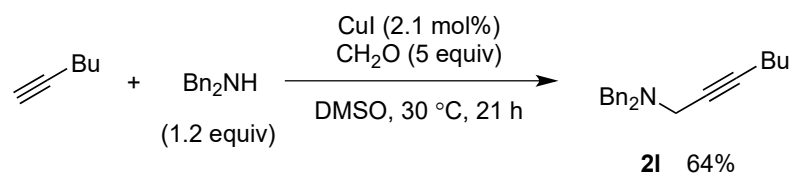

To a dried $100 \mathrm{~mL}$ flask were added CuI (40.0 mg, $0.210 \mathrm{mmol})$, dibenzylamine (2.30 mL, $12.0 \mathrm{mmol})$ and DMSO (20 mL). To the resulting mixture were added formaldehyde solution $(37 \%, 4.00 \mathrm{~mL})$ and 1-hexyne $(1.15 \mathrm{~mL}, 10.0 \mathrm{mmol})$. The resulting mixture was stirred at $30{ }^{\circ} \mathrm{C}$ for $21 \mathrm{~h}$. The reaction was quenched with water, and extracted three times with ether. The combined organic layer was washed with brine, and dried over $\mathrm{Na}_{2} \mathrm{SO}_{4}$. After filtration and evaporation, the residue was purified by silica gel column chromatography (eluent: hexane/AcOEt $=19 / 1 \rightarrow 9 / 1)$ to give $\mathbf{2 l}$ as colorless oil $(1.85 \mathrm{~g}, 6.35$ mmol, 64\%). The spectral data were identical with the reported value. ${ }^{6}$

\section{5-Iodo-1-pentene (3c)}

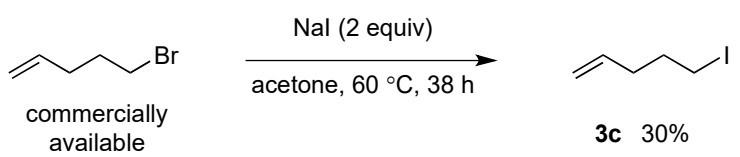

To a dried screw cap tube were added 5-bromo-1-pentene $(1.18 \mathrm{~mL}, 10.0 \mathrm{mmol})$, NaI (3.00 g, $20.0 \mathrm{mmol})$ and acetone (20 $\mathrm{mL}$ ). After the tube was sealed with a cap, the resulting mixture was stirred at $60{ }^{\circ} \mathrm{C}$ for $38 \mathrm{~h}$. After filtration and evaporation, the residue was diluted with ether, washed successively with water and brine, and dried over $\mathrm{Na}_{2} \mathrm{SO}_{4}$. After filtration and evaporation, the residue was purified by silica gel column chromatography (eluent: hexane) to give $\mathbf{3 c}$ as colorless oil (0.593 g, $3.03 \mathrm{mmol}, 30 \%)$. The yield was decreased because $3 \mathbf{c}$ was volatile under reduced pressure. The spectral data were identical with the reported value. ${ }^{7}$

\section{Ethyl 4-iodobutyrate (3d)}

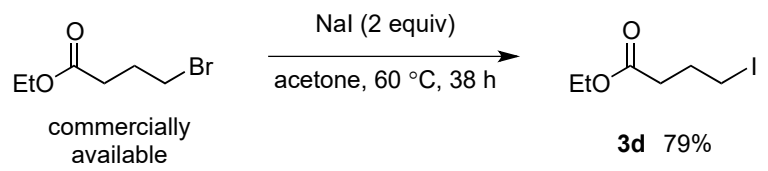

To a dried screw cap tube were added ethyl 4-bromobutyrate $(0.72 \mathrm{~mL}, 5.0 \mathrm{mmol})$, NaI (1.5 g, $10 \mathrm{mmol})$ and acetone (10 $\mathrm{mL}$ ). After the tube was sealed with a cap, the resulting mixture was stirred at $60{ }^{\circ} \mathrm{C}$ for $37 \mathrm{~h}$. After filtration and evaporation, the residue was diluted with ether, washed successively with water and brine, and dried over $\mathrm{Na}_{2} \mathrm{SO}_{4}$. After filtration and evaporation, the residue was purified by silica gel column chromatography (eluent: hexane/AcOEt $=19 / 1$ ) to give 3d as colorless oil (0.95 g, $3.9 \mathrm{mmol}, 79 \%)$. The spectral data were identical with the reported value. ${ }^{8}$ 


\section{Synthesis of ${ }^{\mathrm{NQ}} \mathrm{IMesCuCl}$}<smiles>Cn1c[n+](C)c2c1C(=O)c1ccccc1C2=O</smiles>

$\mathrm{Cl}^{-}$

$\mathrm{NQ}_{\mathrm{IMes}} \cdot \mathrm{HCl}$

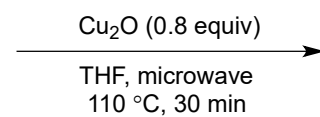

$110^{\circ} \mathrm{C}, 30 \mathrm{~min}$

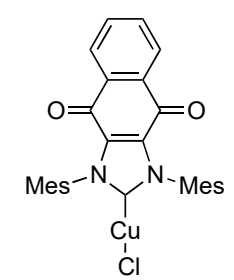

${ }^{\mathrm{NQ}}{ }_{\mathrm{MesCuCl}} \quad 86 \%$

${ }^{\mathrm{NQ}} \mathrm{IMes} \bullet \mathrm{HCl}$ was synthesized according to the reported procedure. ${ }^{9}$ To a dried $0.5-2 \mathrm{~mL}$ microwave vessel were added

${ }^{\mathrm{NQ}} \mathrm{IMes} \bullet \mathrm{HCl}(0.24 \mathrm{~g}, 0.51 \mathrm{mmol}), \mathrm{Cu}_{2} \mathrm{O}(0.057 \mathrm{~g}, 0.40 \mathrm{mmol})$ and THF $(4 \mathrm{~mL})$. The resulting mixture was reacted in the microwave reactor for $30 \mathrm{~min}$ at $110^{\circ} \mathrm{C}$. The reaction mixture was filtrated through a short pad of celite (eluted with DCM). After evaporation, the residue was redissolved in DCM. The resulting red solution was diluted with pentane to give a yellow precipitation. After filtration, ${ }^{\mathrm{NQ}} \mathrm{IMesCuCl}$ was obtained as yellow powder $(0.22 \mathrm{~g}, 0.44 \mathrm{mmol}, 86 \%)$.

${ }^{1} \mathrm{H} \mathrm{NMR}\left(500 \mathrm{MHz}, \mathrm{CDCl}_{3}\right): \delta 8.08(\mathrm{dd}, J=3.2 \mathrm{~Hz}, 5.8 \mathrm{~Hz}, 2 \mathrm{H}), 7.78(\mathrm{dd}, J=3.2 \mathrm{~Hz}, 5.8 \mathrm{~Hz}, 2 \mathrm{H}), 7.08(\mathrm{~s}, 4 \mathrm{H}), 2.40(\mathrm{~s}$, 6H), 2.09 (s, 12H); ${ }^{13} \mathrm{C}$ NMR (125 MHz, $\left.\mathrm{CDCl}_{3}\right): \delta$ 188.5, 174.3, 140.3, 134.8, 133.6, 133.0, 132.0, 129.8, 127.2, 21.3, 17.9; IR (neat, $\mathrm{cm}^{-1}$ ): 3343, 2987, 2918, 1727, 1678, 1589, 1570, 1481, 1411, 1321, 1305, 1264, 1217, 1194, 1072, 1032, 954, 941, 853, 793, 754, 721, 691, 664; HRMS (ESI): $m / z$ calcd for $\mathrm{C}_{29} \mathrm{H}_{26} \mathrm{ClCuN}_{2} \mathrm{NaO}_{2}{ }^{+}\left[\mathrm{M}+\mathrm{Na}^{+}\right]$: 555.0871. Found: 555.0860 .

\section{Optimization Study: Base Effect and Solvent Effect ${ }^{a}$}

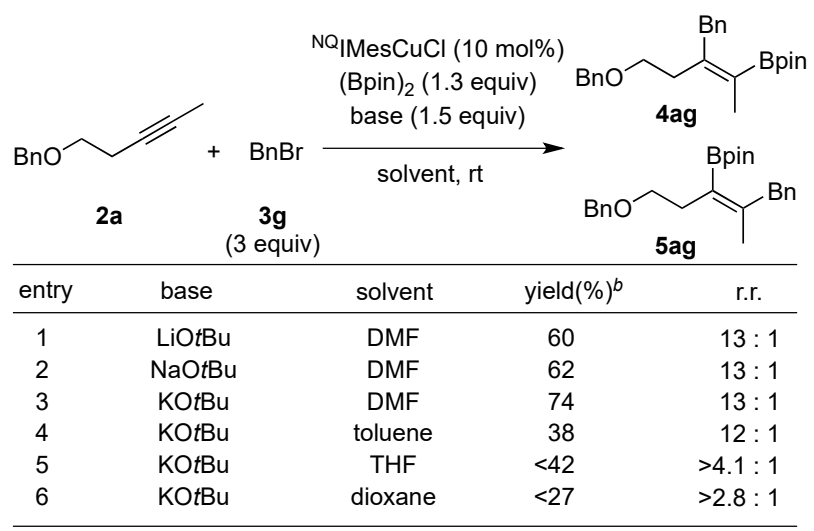

${ }^{a}$ General reaction conditions: $2 \mathrm{a}(0.10 \mathrm{mmol}), \mathbf{3 g}$ (3 equiv), ${ }^{\mathrm{NQ}} \mathrm{IMesCuCl}(10 \mathrm{~mol} \%)$, (Bpin) 2 (1.3 equiv), and base $(1.5$ equiv) in solvent $(0.5 \mathrm{~mL})$ at $\mathrm{rt}$ for $4 \mathrm{~h}$. Yield and regioisomeric ratio (r.r. $=\mathbf{4 a g}: \mathbf{5 a g})$ were determined by ${ }^{1} \mathrm{H}$ NMR analysis of a crude mixture using 1,1,2,2-tetrachloroethane as an internal standard. ${ }^{b}$ Combined yield of $\mathbf{4 a g}$ and $\mathbf{5 a g}$.

DMF was the optimum solvent regarding both product yield and regioselectivity. The base did not affect regioselectivity, but $\mathrm{KO} t \mathrm{Bu}$ produced the highest reactivity. 


\section{Copper-Catalyzed Regioselective Borylalkylation of Dialkylsubstituted Internal Alkynes}

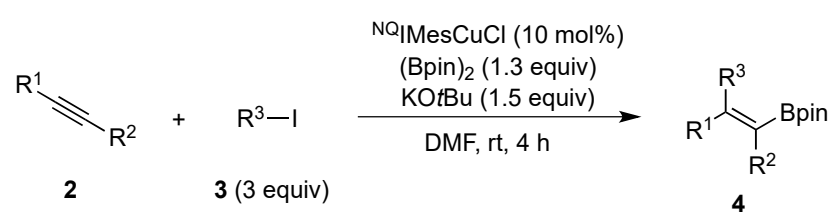

${ }^{\mathrm{NQ}} \mathrm{IMesCuCl}(10.6 \mathrm{mg}, 0.0200 \mathrm{mmol})$ and $(\text { Bpin })_{2}(66.0 \mathrm{mg}, 0.260 \mathrm{mmol})$ were weighed and added to a test tube under air. The test tube was transferred to an Ar filled glove box. $\mathrm{KO} t \mathrm{Bu}(33.6 \mathrm{mg}, 0.300 \mathrm{mmol})$ was added to the test tube in the glove box. The test tube was sealed with a rubber septum, and removed from the glove box. Dehydrated DMF (1.0 mL, commercially available from Kanto Chemical Co., Inc.) was added by syringe. The resulting mixture was stirred at $0{ }^{\circ} \mathrm{C}$ for $10 \mathrm{~min}$, before $2(0.200 \mathrm{mmol})$ and $3(0.600 \mathrm{mmol})$ were added. The resulting mixture was stirred at ambient temperature with water bath for $4 \mathrm{~h}$. The reaction was quenched with sat. aq. $\mathrm{NH}_{4} \mathrm{Cl}$, extracted three times with hexane/AcOEt $=4 / 1$, and dried over $\mathrm{Na}_{2} \mathrm{SO}_{4}$. After filtration and evaporation, the residue was purified by silica gel column chromatography to give a mixture of $\mathbf{4}$ and $\mathbf{5}$, with $\mathbf{4}$ as the major isomer. The stereochemistry of $\mathbf{4}$ was assigned based on the NOE measurement (see below).

(Z)-2-(3-(2-(benzyloxy)ethyl)hept-2-en-2-yl)-4,4,5,5-tetramethyl-1,3,2-dioxaborolane (4aa)<smiles>CC(CCOBr)=C(Br)CBr</smiles>

2a $(36.0 \mu \mathrm{L}, 0.200 \mathrm{mmol})$ and $\mathbf{3 a}(68.0 \mu \mathrm{L}, 0.600 \mathrm{mmol})$ were used to give the crude product $(\mathrm{r} . \mathrm{r} .=10.2: 1)$. The crude product was purified by silica gel column chromatography (eluent: hexane/AcOEt $=49 / 1$ ) to give an inseparable mixture of 4aa and 5aa as colorless oil (64.5 mg, $0.180 \mathrm{mmol}, 90 \%$, r.r. $=9.7: 1)$.

$\mathrm{R}_{\mathrm{f}}=0.30$ (twice with hexane/AcOEt $\left.=30 / 1\right) ;{ }^{1} \mathrm{H} \mathrm{NMR}\left(500 \mathrm{MHz}, \mathrm{CDCl}_{3}\right): \delta 7.36-7.25(\mathrm{~m}, 5 \mathrm{H}, \mathbf{4 a a}+\mathbf{5 a a}), 4.51(\mathrm{~s}, 2 \mathrm{H}$, 4aa+5aa), $3.47(\mathrm{t}, J=7.9 \mathrm{~Hz}, 2 \mathrm{H}, \mathbf{4 a a}), 3.39(\mathrm{t}, J=7.9 \mathrm{~Hz}, 2 \mathrm{H}, \mathbf{5 a a}), 2.48(\mathrm{t}, J=7.9 \mathrm{~Hz}, 2 \mathrm{H}, \mathbf{4 a a}+\mathbf{5 a a}), 2.29(\mathrm{t}, J=7.7 \mathrm{~Hz}$ $2 \mathrm{H}, \mathbf{4 a a}+5 \mathbf{a a}), 1.74(\mathrm{~s}, 3 \mathrm{H}, \mathbf{5 a a}), 1.59$ (s, 3H, 4aa), 1.38-1.22 (m, 16H, 4aa+5aa), $0.88(\mathrm{t}, J=7.1 \mathrm{~Hz}, 3 \mathrm{H}, 4 \mathbf{4 a a}+5 \mathbf{a a}) ;{ }^{13} \mathrm{C}$ NMR (125 MHz, $\left.\mathrm{CDCl}_{3}\right): \delta 151.5,138.5,128.3,127.6,127.4,82.7,72.8,68.4,36.2,33.2,32.3,24.7,24.7,22.8,16.3,14.0$ (The carbon directly attached to the boron atom was not detected due to quadrupolar relaxation); IR (neat, $\mathrm{cm}^{-1}$ ): 3420,2972 , 2959, 2928, 2857, 2359, 1623, 1455, 1357, 1286, 1209, 1147, 1089, 961, 854, 734, 696; HRMS (ESI): $\mathrm{m} / \mathrm{z}$ calcd for $\mathrm{C}_{22} \mathrm{H}_{35} \mathrm{BNaO}_{3}^{+}\left[\mathrm{M}+\mathrm{Na}^{+}\right]: 381.2571$. Found: 381.2560 .

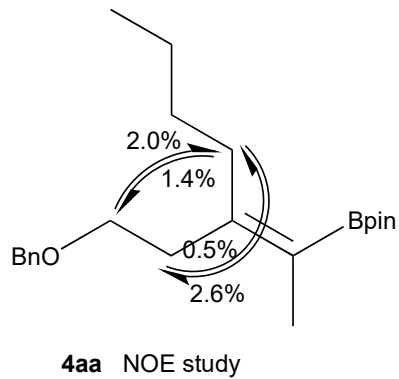


<smiles>CC(=C(Br)Br)C(C)=C(Br)Br</smiles>

2b $(29.0 \mu \mathrm{L}, 0.200 \mathrm{mmol})$ and $\mathbf{3 a}(68.0 \mu \mathrm{L}, 0.600 \mathrm{mmol})$ were used to give the crude product (r.r. $=6.9: 1)$. The crude product was purified by silica gel column chromatography (eluent: hexane/AcOEt $=99 / 1)$ to give an inseparable mixture of 4ba and $5 \mathbf{b a}$ as colorless oil $(43.5 \mathrm{mg}, 0.147 \mathrm{mmol}, 74 \%$, r.r. $=9.0: 1)$.

$\mathrm{R}_{\mathrm{f}}=0.40$ (twice with hexane/AcOEt $\left.=30 / 1\right) ;{ }^{1} \mathrm{H} \mathrm{NMR}\left(500 \mathrm{MHz}, \mathrm{CDCl}_{3}\right): \delta 2.28(\mathrm{t}, J=7.4 \mathrm{~Hz}, 2 \mathrm{H}, \mathbf{4 b a}+\mathbf{5 b a}), 2.07$ (t, 7.9 Hz, 2H, 4ba+5ba), 1.69 (s, 3H, 5ba), 1.67 (s, 3H, 4ba), 1.37-1.24 (m, 10H, 4ba+5ba), 1.27 (s, 12H. 4ba+5ba) 0.91-0.87 (m, $6 \mathrm{H}, 4 \mathbf{b a}+\mathbf{5 b a}) ;{ }^{13} \mathrm{C}$ NMR $\left(125 \mathrm{MHz}, \mathrm{CDCl}_{3}\right): \delta 156.6,82.6,35.7,32.7,32.4,32.3,27.7,24.8,22.9,22.6,16.1,14.0$ (The carbon directly attached to the boron atom was not detected due to quadrupolar relaxation); IR (neat, $\mathrm{cm}^{-1}$ ): 3466,2972 , 2957, 2929, 2859, 1619, 1540, 1507, 1465, 1357, 1284, 1213, 1148, 1092, 966, 856, 691, 670; HRMS (DART): $m / z$ calcd for $\mathrm{C}_{18} \mathrm{H}_{36} \mathrm{BO}_{2}^{+}\left[\mathrm{M}+\mathrm{H}^{+}\right]: 295.2803$. Found: 295.2793.

(Z)-2-(3-isopropylhept-2-en-2-yl)-4,4,5,5-tetramethyl-1,3,2-dioxaborolane (4ca)<smiles>CC(=C(Br)Br)C(C)C</smiles>

2c $(23.0 \mu \mathrm{L}, 0.200 \mathrm{mmol})$ and 3a $(68.0 \mu \mathrm{L}, 0.600 \mathrm{mmol})$ were used to give the crude product (r.r. could not be determined due to overlapped impurities). The crude product was purified by silica gel column chromatography (eluent: hexane/AcOEt $=49 / 1)$ and GPC to give an inseparable mixture of $\mathbf{4} \mathbf{c a}$ and $\mathbf{5 c a}$ as colorless oil $(27.0 \mathrm{mg}, 0.101 \mathrm{mmol}, 51 \%$, r.r. $=17.2: 1)$. $\mathrm{R}_{\mathrm{f}}=0.51$ (twice with hexane/AcOEt $\left.=30 / 1\right) ;{ }^{1} \mathrm{H}$ NMR $\left(500 \mathrm{MHz}, \mathrm{CDCl}_{3}\right): \delta 2.93$ (sept, $\left.J=6.9 \mathrm{~Hz}, 1 \mathrm{H}, 4 \mathrm{ca}\right), 2.65$ (sept, $J=$ $6.9 \mathrm{~Hz}, 1 \mathrm{H}, \mathbf{5 c a}), 2.20$ (t, $J=7.9 \mathrm{~Hz}, 2 \mathrm{H}, \mathbf{4 c a}), 2.08$ (t, $J=7.7 \mathrm{~Hz}, 2 \mathrm{H}, \mathbf{5 c a}), 1.69$ (s, 3H, 4ca), 1.66 (s, 3H, 5ca), 1.39-1.19 $(\mathrm{m}, 4 \mathrm{H}, \mathbf{4 c a}+\mathbf{5 c a}), 1.26(\mathrm{~s}, 12 \mathrm{H}, \mathbf{4 c a}+\mathbf{5 c a}), 1.01(\mathrm{~d}, J=6.9 \mathrm{~Hz}, 6 \mathrm{H}, \mathbf{5 c a}), 0.98(\mathrm{~d}, J=6.9 \mathrm{~Hz}, 6 \mathrm{H}, \mathbf{4 c a}), 0.89$ (t, $J=7.0 \mathrm{~Hz}$ $3 \mathrm{H}, \mathbf{4 c a}+\mathbf{5 c a}) ;{ }^{13} \mathrm{C}$ NMR $\left(125 \mathrm{MHz}, \mathrm{CDCl}_{3}\right): \delta 160.5,82.6,35.2,31.1,30.6,24.8,23.5,20.8,15.5,14.0$ (The carbon directly attached to the boron atom was not detected due to quadrupolar relaxation); IR (neat, $\mathrm{cm}^{-1}$ ): 2959, 2929, 2872, 1607, 1457 , 1375, 1353, 1282, 1146, 1086, 966, 865, 765, 730; HRMS (DART): $m / z$ calcd for $\mathrm{C}_{16} \mathrm{H}_{32} \mathrm{BO}_{2}^{+}\left[\mathrm{M}+\mathrm{H}^{+}\right]: 267.2490$. Found: 267.2502 .

\section{(E)-2-(4-ethyloct-3-en-3-yl)-4,4,5,5-tetramethyl-1,3,2-dioxaborolane (4da)}<smiles>CC/C(Br)=C(\Br)CC</smiles>

2d $(23.0 \mu \mathrm{L}, 0.200 \mathrm{mmol})$ and 3a $(68.0 \mu \mathrm{L}, 0.600 \mathrm{mmol})$ were used to give the crude product. The crude product was purified by silica gel column chromatography (eluent: hexane/AcOEt $=99 / 1)$ to give pure 4da as colorless oil $(44.0 \mathrm{mg}$, $0.165 \mathrm{mmol}, 83 \%)$.

$\mathrm{R}_{\mathrm{f}}=0.44$ (twice with hexane/AcOEt $\left.=30 / 1\right) ;{ }^{1} \mathrm{H}$ NMR $\left(500 \mathrm{MHz}, \mathrm{CDCl}_{3}\right): \delta 2.25(\mathrm{t}, J=7.6 \mathrm{~Hz}, 2 \mathrm{H}), 2.14-2.07(\mathrm{~m}, 4 \mathrm{H})$, 1.36-1.24 (m, 4H), $1.28(\mathrm{~s}, 12 \mathrm{H}), 0.97(\mathrm{dt}, J=0.9 \mathrm{~Hz}, 7.6 \mathrm{~Hz}, 3 \mathrm{H}), 0.93(\mathrm{dt}, J=0.9 \mathrm{~Hz}, 7.6 \mathrm{~Hz}, 3 \mathrm{H}), 0.89(\mathrm{t}, J=7.1 \mathrm{~Hz}$, 
$3 \mathrm{H}) ;{ }^{13} \mathrm{C}$ NMR $\left(125 \mathrm{MHz}, \mathrm{CDCl}_{3}\right.$ ): $\delta 155.3,82.6,35.3,32.4,24.8,24.7,23.7,23.0,15.2,14.0,13.5$ (The carbon directly attached to the boron atom was not detected due to quadrupolar relaxation); IR (neat, $\mathrm{cm}^{-1}$ ): 3420, 3011, 2963, 2931, 2871, $1617,1466,1389,1372,1358,1318,1284,1256,1215,1146,1109,1036,965,927,869,852,760,668$; HRMS (DART): $m / z$ calcd for $\mathrm{C}_{16} \mathrm{H}_{32} \mathrm{BO}_{2}^{+}\left[\mathrm{M}+\mathrm{H}^{+}\right]: 267.2490$. Found: 267.2499 .

(Z)-tert-butyldimethyl((3-(1-(4,4,5,5-tetramethyl-1,3,2-dioxaborolan-2-yl)ethylidene)heptyl)oxy)silane (4ea)<smiles>CC(CCC(C)=C(Cc1ccccc1)c1ccccc1)=C(Br)CCOC(C)(C)C</smiles>

2e $(48.0 \mu \mathrm{L}, 0.200 \mathrm{mmol})$ and $3 \mathbf{a}(68.0 \mu \mathrm{L}, 0.600 \mathrm{mmol})$ were used to give the crude product $(\mathrm{r} . \mathrm{r} .=11.6: 1)$. The crude product was purified by silica gel column chromatography (eluent: hexane/AcOEt $=49 / 1$ ) to give an inseparable mixture of 4ea and 5ea as colorless oil $(69.7 \mathrm{mg}, 0.182 \mathrm{mmol}, 91 \%$, r.r. $=9.8: 1)$.

$\mathrm{R}_{\mathrm{f}}=0.43$ (twice with hexane/AcOEt $\left.=30 / 1\right) ;{ }^{1} \mathrm{H} \mathrm{NMR}\left(500 \mathrm{MHz}, \mathrm{CDCl}_{3}\right): \delta 3.59(\mathrm{t}, J=8.0 \mathrm{~Hz}, 2 \mathrm{H}, 4 \mathrm{ea}), 3.51(\mathrm{t}, J=8.0 \mathrm{~Hz}$, 2H, 5ea), 2.38 (t, $J=8.0 \mathrm{~Hz}, 2 \mathrm{H}, 4 \mathbf{e a}+5 \mathbf{e a}), 2.28$ (t, $J=7.6 \mathrm{~Hz}, 2 \mathrm{H}, 4 \mathbf{e a}+5 \mathbf{e a}), 1.75$ (s, 3H, 5ea), 1.69 (s, 3H, 4ea), 1.38-1.22 (m, 16H, 4ea+5ea), 0.89 (t, $J=7.2 \mathrm{~Hz}, 3 \mathrm{H}, \mathbf{4 e a}+5 \mathbf{e a}), 0.89$ (s, 9H, 4ea+5ea), 0.05 (s, 6H, 4ea+5ea); ${ }^{13} \mathrm{C}$ NMR $(125 \mathrm{MHz}$, $\mathrm{CDCl}_{3}$ ): $\delta 151.7,82.7,61.5,36.6,36.3,32.2,26.0,24.7,22.8,18.3,16.3,14.0,-5.2$ (The carbon directly attached to the boron atom was not detected due to quadrupolar relaxation); IR (neat, $\mathrm{cm}^{-1}$ ): 3446, 3018, 2957, 2929, 2857, 2399, 1620, $1520,1470,1358,1285,1255,1215,1146,1082,1005,964,929,836,756,691,669,626$; HRMS (ESI): $\mathrm{m} / z$ calcd for $\mathrm{C}_{21} \mathrm{H}_{43} \mathrm{BNaO}_{3} \mathrm{Si}^{+}\left[\mathrm{M}+\mathrm{Na}^{+}\right]$: 405.2967. Found: 405.2971.

(Z)-3-(1-(4,4,5,5-tetramethyl-1,3,2-dioxaborolan-2-yl)ethylidene)heptyl pivalate (4fa)<smiles>C/C(Cc1ccccc1)=C(\Br)CCOP</smiles>

2f $(38.0 \mu \mathrm{L}, 0.200 \mathrm{mmol})$ and 3a $(68.0 \mu \mathrm{L}, 0.600 \mathrm{mmol})$ were used to give the crude product $($ r.r. $=8.1: 1)$. The crude product was purified by silica gel column chromatography (eluent: hexane/AcOEt $=49 / 1)$ to give an inseparable mixture of 4fa and $\mathbf{5 f a}$ as colorless oil $(57.1 \mathrm{mg}, 0.162 \mathrm{mmol}, 81 \%$, r.r. $=7.5: 1)$.

$\mathrm{R}_{\mathrm{f}}=0.32$ (twice with hexane/AcOEt $\left.=30 / 1\right) ;{ }^{1} \mathrm{H} \mathrm{NMR}\left(500 \mathrm{MHz}, \mathrm{CDCl}_{3}\right): \delta 4.05(\mathrm{t}, J=7.7 \mathrm{~Hz}, 2 \mathrm{H}, 4 \mathbf{f a}), 3.98(\mathrm{t}, J=7.6 \mathrm{~Hz}$, 2H, 4fa), 2.36 (t, $J=7.6 \mathrm{~Hz}, 2 \mathrm{H}, \mathbf{4 f a}), 2.32$ (t, $J=7.7 \mathrm{~Hz}, 2 \mathrm{H}, \mathbf{4 f a}), 1.79$ (s, 3H, 4fa), 1.73 (s, 3H, 4fa), 1.39-1.13 (m, 4H, $\mathbf{4 f a}+\mathbf{5 f a}), 1.26(\mathrm{~s}, 12 \mathrm{H}, \mathbf{4 f a}+\mathbf{5 f a}), 1.19(\mathrm{~s}, 9 \mathrm{H}, \mathbf{4 f a}+\mathbf{5 f a}), 0.90(\mathrm{t}, J=7.1 \mathrm{~Hz}, 3 \mathrm{H}, \mathbf{4 f a}+\mathbf{5 f a}) ;{ }^{13} \mathrm{C} \mathrm{NMR}\left(125 \mathrm{MHz}, \mathrm{CDCl}_{3}\right): \delta$ $178.6,150.4,82.8,62.5,38.6,35.9,32.2,31.8,27.2,27.2,24.7,22.8,16.4,14.0$ (The carbon directly attached to the boron atom was not detected due to quadrupolar relaxation); IR (neat, $\mathrm{cm}^{-1}$ ): 3420, 3019, 2978, 2931, 2872, 2399, 1717, 1622,

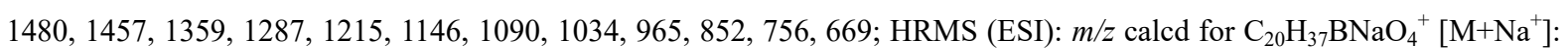
375.2677. Found: 375.2668 . 
(Z)-methyl 5-(1-(4,4,5,5-tetramethyl-1,3,2-dioxaborolan-2-yl)ethylidene)nonanoate (4ga)<smiles>COC(=O)CCC/C(C)=C(\C)Cc1ccccc1</smiles>

$2 \mathbf{g}(29.0 \mu \mathrm{L}, 0.200 \mathrm{mmol})$ and $\mathbf{3 a}(68.0 \mu \mathrm{L}, 0.600 \mathrm{mmol})$ were used to give the crude product (r.r. could not be determined due to overlapped impurities). The crude product was purified by silica gel column chromatography (eluent: hexane/AcOEt $=49 / 1)$ to give an inseparable mixture of $\mathbf{4 g a}$ and $\mathbf{5 g a}$ as colorless oil $(51.5 \mathrm{mg}, 0.159 \mathrm{mmol}, 79 \%$, r.r. $=10.3: 1)$.

$\mathrm{R}_{\mathrm{f}}=0.11$ (twice with hexane/AcOEt $\left.=30 / 1\right) ;{ }^{1} \mathrm{H}$ NMR $\left(500 \mathrm{MHz}, \mathrm{CDCl}_{3}\right): \delta 3.66(\mathrm{~s}, 3 \mathrm{H}, \mathbf{4 g a}), 3.65(\mathrm{~s}, 3 \mathrm{H}, \mathbf{5 g a}), 2.33-2.27$ $(\mathrm{m}, 4 \mathrm{H}, \mathbf{4 g a}+\mathbf{5 g a}), 2.12(\mathrm{t}, J=8.0 \mathrm{~Hz}, 2 \mathrm{H}, \mathbf{4 g a}+\mathbf{5 g a}), 1.67(\mathrm{~s}, 3 \mathrm{H}, \mathbf{4 g a}), 1.64(\mathrm{~s}, 3 \mathrm{H}, \mathbf{5 g a}), 1.35-1.25(\mathrm{~m}, 4 \mathrm{H}, \mathbf{4 g a}+\mathbf{5 g a}), 1.26$ (s, 12H, 4ga+5ga), $0.89(\mathrm{t}, J=7.1 \mathrm{~Hz}, 3 \mathrm{H}, \mathbf{4 g a}+\mathbf{5 g a}) ;{ }^{13} \mathrm{C} \mathrm{NMR}\left(125 \mathrm{MHz}, \mathrm{CDCl}_{3}\right): \delta 174.1,154.6,82.7,51.4,35.4,34.1$, $32.3,31.7,24.8,23.2,22.9,16.1,14.0$ (The carbon directly attached to the boron atom was not detected due to quadrupolar relaxation); IR (neat, $\mathrm{cm}^{-1}$ ): 3446, 3016, 2981, 2955, 2933, 2870, 1732, 1619, 1461, 1437, 1360, 1284, 1215, 1146, 1109, 1088, 965, 860, 756, 690, 668; HRMS (ESI): $m / z$ calcd for $\mathrm{C}_{18} \mathrm{H}_{33} \mathrm{BNaO}_{4}{ }^{+}\left[\mathrm{M}+\mathrm{Na}^{+}\right]$: 347.2364. Found: 347.2361 .

(Z)-N,N-diethyl-5-(1-(4,4,5,5-tetramethyl-1,3,2-dioxaborolan-2-yl)ethylidene)nonanamide (4ha)<smiles>CCNC(=O)CCC/C(Br)=C(\C)Cc1ccccc1</smiles>

2h $(39.0 \mu \mathrm{L}, 0.200 \mathrm{mmol})$ and $\mathbf{3 a}(68.0 \mu \mathrm{L}, 0.600 \mathrm{mmol})$ were used to give the crude product (r.r. could not be determined due to overlapped impurities). The crude product was purified by silica gel column chromatography (eluent: hexane/AcOEt $=49 / 1)$ to give pure $4 \mathrm{ha}(50.0 \mathrm{mg}, 0.160 \mathrm{mmol})$ and $\mathbf{5 h a}(6.4 \mathrm{mg}, 0.018 \mathrm{mmol})$ as colorless oil $(89 \%$, r.r. $=9.1: 1)$.

4ha: $\mathrm{R}_{\mathrm{f}}=0.36($ hexane/AcOEt $=2 / 1) ;{ }^{1} \mathrm{H} \mathrm{NMR}\left(500 \mathrm{MHz}, \mathrm{CDCl}_{3}\right): \delta 3.36(\mathrm{q}, 2 \mathrm{H}, J=7.1 \mathrm{~Hz}), 3.29(\mathrm{q}, 2 \mathrm{H}, J=7.1 \mathrm{~Hz})$, 2.32-2.28 (m, 4H), 2.15 (t, 2H, $J=8.0 \mathrm{~Hz}), 1.75-1.69(\mathrm{~m}, 2 \mathrm{H}), 1.68(\mathrm{~s}, 3 \mathrm{H}), 1.36-1.24(\mathrm{~m}, 4 \mathrm{H}), 1.26(\mathrm{~s}, 12 \mathrm{H}), 1.16(\mathrm{t}, 3 \mathrm{H}, J$ $=7.1 \mathrm{~Hz}), 1.10(\mathrm{t}, 3 \mathrm{H}, J=7.1 \mathrm{~Hz}), 0.89(\mathrm{t}, 3 \mathrm{H}, J=7.1 \mathrm{~Hz}) ;{ }^{13} \mathrm{C} \mathrm{NMR}\left(125 \mathrm{MHz}, \mathrm{CDCl}_{3}\right): \delta 171.9,155.4,82.6,41.9,39.9$, $35.5,33.1,32.4,32.1,24.8,23.7,22.9,16.2,14.3,14.0,13.1$ (The carbon directly attached to the boron atom was not detected due to quadrupolar relaxation); IR (neat, $\mathrm{cm}^{-1}$ ): 3420, 2975, 2929, 2872, 1644, 1457, 1431, 1360, 1283, 1217, 1146, 1087, 966, 861, 754, 687, 665; HRMS (ESI): $\mathrm{m} / z$ calcd for $\mathrm{C}_{21} \mathrm{H}_{40} \mathrm{BNNaO}_{3}{ }^{+}\left[\mathrm{M}+\mathrm{Na}^{+}\right]$: 388.2993. Found: 388.2980 .

5ha: $\mathrm{R}_{\mathrm{f}}=0.25($ hexane/AcOEt $=2 / 1) ;{ }^{1} \mathrm{H}$ NMR $\left(500 \mathrm{MHz}, \mathrm{CDCl}_{3}\right): \delta 3.35(\mathrm{q}, J=7.1 \mathrm{~Hz}, 2 \mathrm{H}), 3.28(\mathrm{q}, J=7.1 \mathrm{~Hz}, 2 \mathrm{H})$, 2.32-2.26 (m, 4H), $2.16(\mathrm{t}, J=7.6 \mathrm{~Hz}, 2 \mathrm{H}), 1.72(\mathrm{~s}, 3 \mathrm{H}), 1.68-1.62(\mathrm{~m}, 2 \mathrm{H}), 1.38-1.24(\mathrm{~m}, 4 \mathrm{H}), 1.25(\mathrm{~s}, 12 \mathrm{H}), 1.15(\mathrm{t}, J=7.1$ $\mathrm{Hz}, 3 \mathrm{H}), 1.09(\mathrm{t}, J=7.1 \mathrm{~Hz}, 3 \mathrm{H}), 0.89$ (t, $J=7.2 \mathrm{~Hz}, 3 \mathrm{H}) ;{ }^{13} \mathrm{C} \mathrm{NMR}\left(125 \mathrm{MHz}, \mathrm{CDCl}_{3}\right): \delta 172.5,152.0,82.6,41.9,39.8$, $38.0,33.0,31.7,30.6,25.9,24.8,22.6,18.7,14.3,14.1,13.1$ (The carbon directly attached to the boron atom was not detected due to quadrupolar relaxation); IR (neat, $\mathrm{cm}^{-1}$ ): 3447, 2974, 2930, 2871, 1644, 1458, 1428, 1376, 1352, 1276, 1214 , 1144, 1082, 968, 862, 710; HRMS (ESI): $m / z$ calcd for $\mathrm{C}_{21} \mathrm{H}_{40} \mathrm{BNNaO}_{3}{ }^{+}\left[\mathrm{M}+\mathrm{Na}^{+}\right]$: 388.2993. Found: 388.2980.

(Z)-2-(3-((benzyloxy)methyl)hept-2-en-2-yl)-4,4,5,5-tetramethyl-1,3,2-dioxaborolane (4ia)<smiles>[B]OC/C(C)=C(/Br)Cc1ccccc1</smiles> 
$2 \mathbf{i}(33.0 \mu \mathrm{L}, 0.200 \mathrm{mmol})$ and $3 \mathrm{a}(68.0 \mu \mathrm{L}, 0.600 \mathrm{mmol})$ were used to give the crude product (r.r. $=>20: 1)$. The crude product was purified by silica gel column chromatography (eluent: hexane/AcOEt $=49 / 1$ ) to give pure 4ia as colorless oil (59.2 mg, $0.163 \mathrm{mmol}, 82 \%)$.

$\mathrm{R}_{\mathrm{f}}=0.28$ (twice with hexane/AcOEt $\left.=30 / 1\right) ;{ }^{1} \mathrm{H}$ NMR $\left(500 \mathrm{MHz}, \mathrm{CDCl}_{3}\right): \delta 7.35-7.31(\mathrm{~m}, 4 \mathrm{H}), 7.28-7.25(\mathrm{~m}, 1 \mathrm{H}), 4.47(\mathrm{~s}$, $2 \mathrm{H}), 4.08(\mathrm{~s}, 2 \mathrm{H}), 2.42(\mathrm{t}, J=7.8 \mathrm{~Hz}, 2 \mathrm{H}), 1.71(\mathrm{~s}, 3 \mathrm{H}), 1.41-1.36(\mathrm{~m}, 2 \mathrm{H}), 1.33-1.28(\mathrm{~m}, 2 \mathrm{H}), 1.27(\mathrm{~s}, 12 \mathrm{H}), 0.89(\mathrm{t}, J=7.2$ $\mathrm{Hz}, 3 \mathrm{H}) ;{ }^{13} \mathrm{C} \mathrm{NMR}\left(125 \mathrm{MHz}, \mathrm{CDCl}_{3}\right): \delta 150.9,138.7,128.2,127.6,127.4,82.9,72.1,68.5,33.6,32.2,24.8,22.9,15.9$, 14.0 (The carbon directly attached to the boron atom was not detected due to quadrupolar relaxation); IR (neat, $\mathrm{cm}^{-1}$ ): 3420 , 2972, 2955, 2929, 2858, 1716, 1623, 1540, 1507, 1455, 1353, 1294, 1213, 1146, 1073, 965, 853, 734, 697; HRMS (ESI): $m / z$ calcd for $\mathrm{C}_{21} \mathrm{H}_{33} \mathrm{BNaO}_{3}{ }^{+}\left[\mathrm{M}+\mathrm{Na}^{+}\right]: 367.2415$. Found: 267.2401 .

(Z)-2-(4-((benzyloxy)methyl)oct-3-en-3-yl)-4,4,5,5-tetramethyl-1,3,2-dioxaborolane (4ja)<smiles>CC/C(CBr)=C(\Br)COCc1ccccc1</smiles>

$2 \mathbf{j}(36.0 \mu \mathrm{L}, 0.200 \mathrm{mmol})$ and $\mathbf{3 a}(68.0 \mu \mathrm{L}, 0.600 \mathrm{mmol})$ were used to give the crude product (r.r. could not be determined due to overlapped impurities). The crude product was purified by silica gel column chromatography (eluent: hexane/AcOEt $=49 / 1)$ to give an inseparable mixture of $\mathbf{4 j a}$ and $\mathbf{5 j a}$ as colorless oil $(50.0 \mathrm{mg}, 0.139 \mathrm{mmol}, 70 \%$, r.r. $=11.9: 1)$.

$\mathrm{R}_{\mathrm{f}}=0.22$ (twice with hexane/AcOEt $\left.=30 / 1\right) ;{ }^{1} \mathrm{H} \mathrm{NMR}\left(500 \mathrm{MHz}, \mathrm{CDCl}_{3}\right): \delta 7.37-7.24(\mathrm{~m}, 5 \mathrm{H}, \mathbf{4} \mathbf{j a}+\mathbf{5 j a}), 4.49(\mathrm{~s}, 2 \mathrm{H}, \mathbf{5 j a})$, 4.46 (s, 2H, 4ja), 4.14 (s, 2H, 5ja), 4.07 (s, 2H, 4ja), 2.38 (t, $J=7.8$ Hz, 2H, 4ja), 2.26 (t, $J=7.9$ Hz, 2H, 5ja), 2.16 (q, $J=$ $7.4 \mathrm{~Hz}, 2 \mathrm{H}, \mathbf{4 j a}), 2.11$ (q, $J=7.7 \mathrm{~Hz}, 2 \mathrm{H}, \mathbf{5 j a}), 1.42-1.36(\mathrm{~m}, 2 \mathrm{H}, \mathbf{4 j a} \mathbf{5} \mathbf{5 j a}), 1.34-1.21$ (m, 14H, 4ja $\mathbf{5} \mathbf{5 j a}), 0.91(\mathrm{t}, J=7.4 \mathrm{~Hz}$, $3 \mathrm{H}, \mathbf{4 j a}+\mathbf{5 j a}), 0.89(\mathrm{t}, J=7.1 \mathrm{~Hz}, 3 \mathrm{H}, \mathbf{4 j a} \mathbf{\mathbf { a }}+\mathbf{5 j a}) ;{ }^{13} \mathrm{C} \mathrm{NMR}\left(125 \mathrm{MHz}, \mathrm{CDCl}_{3}\right): \delta 148.7,138.7,128.2,127.6,127.4,82.9$, $71.9,67.8,33.8,32.2,24.8,23.6,22.9,15.1,14.0$ (The carbon directly attached to the boron atom was not detected due to quadrupolar relaxation); IR (neat, $\mathrm{cm}^{-1}$ ): 3412, 2958, 2924, 2868, 2367, 2319, 1652, 1540, 1507, 1456, 1360, 1288, 1145, 1091, 966, 857, 769; HRMS (ESI): $m / z$ calcd for $\mathrm{C}_{22} \mathrm{H}_{35} \mathrm{BNaO}_{3}{ }^{+}\left[\mathrm{M}+\mathrm{Na}^{+}\right]: 381.2571$. Found: 381.2575 .

\section{(Z)-2-(4-(2-(benzyloxy)ethyl)oct-3-en-3-yl)-4,4,5,5-tetramethyl-1,3,2-dioxaborolane (4ka)}<smiles>CC/C(Cc1ccccc1)=C(\Br)CCOCc1ccccc1</smiles>

$\mathbf{2 k}(39.0 \mu \mathrm{L}, 0.200 \mathrm{mmol})$ and $\mathbf{3 a}(68.0 \mu \mathrm{L}, 0.600 \mathrm{mmol})$ were used to give the crude product (r.r. $=1.7: 1)$. The crude product was purified by silica gel column chromatography (eluent: hexane/AcOEt $=49 / 1$ ) to give a mixture of $4 \mathbf{k a}$ and $\mathbf{5 k a}$ as colorless oil (66.1 mg, $0.177 \mathrm{mmol}, 89 \%$, r.r. = $1.8: 1)$. Pure 4ka and 5ka were isolated by GPC.

4ka: $\mathrm{R}_{\mathrm{f}}=0.23$ (twice with hexane/AcOEt $\left.=30 / 1\right) ;{ }^{1} \mathrm{H} \mathrm{NMR}\left(500 \mathrm{MHz}, \mathrm{CDCl}_{3}\right): \delta 7.33(\mathrm{~d}, J=4.6 \mathrm{~Hz}, 4 \mathrm{H}), 7.28-7.25(\mathrm{~m}$, $1 \mathrm{H}), 4.50(\mathrm{~s}, 2 \mathrm{H}), 3.46(\mathrm{t}, J=8.0 \mathrm{~Hz}, 2 \mathrm{H}), 2.46(\mathrm{t}, J=8.0 \mathrm{~Hz}, 2 \mathrm{H}), 2.23(\mathrm{t}, J=7.9 \mathrm{~Hz}, 2 \mathrm{H}), 2.12(\mathrm{q}, J=7.4 \mathrm{~Hz}, 2 \mathrm{H})$, $1.38-1.25(\mathrm{~m}, 4 \mathrm{H}), 1.26(\mathrm{~s}, 12 \mathrm{H}), 0.91(\mathrm{t}, J=7.4 \mathrm{~Hz}, 3 \mathrm{H}), 0.88(\mathrm{t}, J=7.1 \mathrm{~Hz}, 3 \mathrm{H}) ;{ }^{13} \mathrm{C}^{\mathrm{NMR}}\left(125 \mathrm{MHz}, \mathrm{CDCl}_{3}\right): \delta 149.0$, $138.6,128.4,127.7,127.6,82.9,72.9,69.4,36.5,32.6,32.5,24.9,24.2,23.0,15.2,14.2$ (The carbon directly attached to the boron atom was not detected due to quadrupolar relaxation); IR (neat, $\mathrm{cm}^{-1}$ ): 3446, 2972, 2958, 2928, 2868, 1617, 1455, 


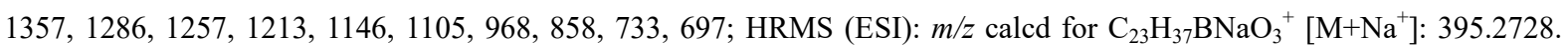
Found: 395.2732 .

5ka: $\mathrm{R}_{\mathrm{f}}=0.23$ (twice with hexane/AcOEt $\left.=30 / 1\right) ;{ }^{1} \mathrm{H} \mathrm{NMR}\left(500 \mathrm{MHz}, \mathrm{CDCl}_{3}\right): \delta 7.35-7.30(\mathrm{~m}, 4 \mathrm{H}), 7.26-7.25(\mathrm{~m}, 1 \mathrm{H})$, $4.51(\mathrm{~s}, 2 \mathrm{H}), 3.39$ (t, $J=7.9 \mathrm{~Hz}, 2 \mathrm{H}), 2.47$ (t, $J=7.9 \mathrm{~Hz}, 2 \mathrm{H}), 2.28$ (t, $J=7.7 \mathrm{~Hz}, 2 \mathrm{H}), 2.11$ (q, $J=7.6 \mathrm{~Hz}, 2 \mathrm{H}), 1.34-1.26$ (m, 4H), $1.22(\mathrm{~s}, 12 \mathrm{H}), 0.96(\mathrm{t}, J=7.6 \mathrm{~Hz}, 3 \mathrm{H}), 0.89$ (t, $J=7.2 \mathrm{~Hz}, 3 \mathrm{H}) ;{ }^{13} \mathrm{C}$ NMR $\left(125 \mathrm{MHz}, \mathrm{CDCl}_{3}\right): \delta 159.0,138.8,128.2$, $127.5,127.3,82.6,72.5,70.6,35.1,32.4,31.0,25.1,24.7,22.9,14.0,13.4$ (The carbon directly attached to the boron atom was not detected due to quadrupolar relaxation); IR (neat, $\mathrm{cm}^{-1}$ ): 3419, 2973, 2929, 2863, 1617, 1455, 1359, 1285, 1213, 1146, 1103, 965, 853, 697; HRMS (ESI): $m / z$ calcd for $\mathrm{C}_{23} \mathrm{H}_{37} \mathrm{BNaO}_{3}{ }^{+}\left[\mathrm{M}+\mathrm{Na}^{+}\right]:$395.2728. Found: 395.2732 .
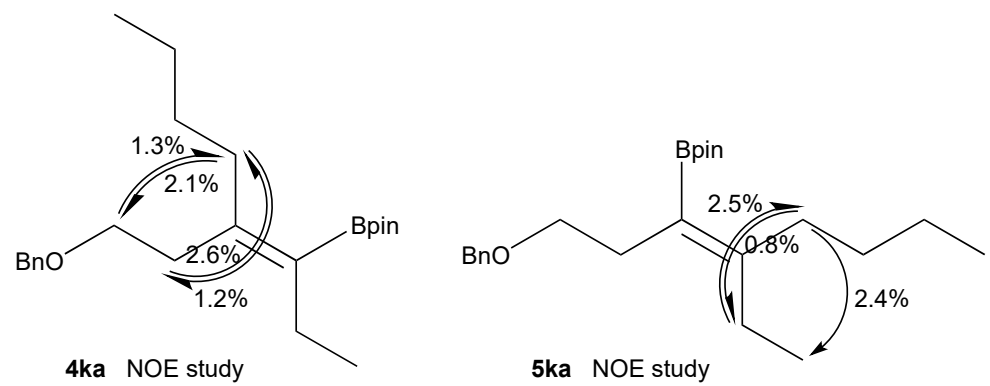

(Z)-N,N-dibenzyl-2-butyl-3-(4,4,5,5-tetramethyl-1,3,2-dioxaborolan-2-yl)hept-2-en-1-amine (4la)<smiles>BrCC(Br)=C(Br)CNCc1ccccc1</smiles>

2l $(59.0 \mu \mathrm{L}, 0.200 \mathrm{mmol})$ and $3 \mathbf{a}(68.0 \mu \mathrm{L}, 0.600 \mathrm{mmol})$ were used to give the crude product (r.r. $=>20: 1)$. The crude product was purified by silica gel column chromatography (eluent: hexane/AcOEt $=19 / 1$ ) to give pure 4la as white solids (62.0 mg, $0.130 \mathrm{mmol}, 65 \%)$.

$\mathrm{R}_{\mathrm{f}}=0.45$ (hexane/AcOEt $\left.=10 / 1\right) ;{ }^{1} \mathrm{H}$ NMR $\left(500 \mathrm{MHz}, \mathrm{CDCl}_{3}\right): \delta 7.37(\mathrm{~d}, J=7.2 \mathrm{~Hz}, 4 \mathrm{H}), 7.29(\mathrm{t}, J=7.6 \mathrm{~Hz}, 4 \mathrm{H}), 7.20(\mathrm{t}, J$ $=7.3 \mathrm{~Hz}, 2 \mathrm{H}), 3.45(\mathrm{~s}, 4 \mathrm{H}), 2.99(\mathrm{~s}, 2 \mathrm{H}), 2.41(\mathrm{t}, J=8.2 \mathrm{~Hz}, 2 \mathrm{H}), 2.19(\mathrm{t}, J=7.6 \mathrm{~Hz}, 2 \mathrm{H}), 1.31-1.20(\mathrm{~m}, 6 \mathrm{H}), 1.24(\mathrm{~s}, 12 \mathrm{H})$, $1.12-1.07(\mathrm{~m}, 2 \mathrm{H}), 0.88(\mathrm{t}, J=7.0 \mathrm{~Hz}, 3 \mathrm{H}), 0.83(\mathrm{t}, J=7.3 \mathrm{~Hz}, 3 \mathrm{H}) ;{ }^{13} \mathrm{C}$ NMR $\left(125 \mathrm{MHz}, \mathrm{CDCl}_{3}\right): \delta 150.1,140.2,128.7$, $128.0,126.6,82.7,58.4,52.7,33.8,32.9,32.1,30.0,24.7,23.1,22.7,14.1,14.0$ (The carbon directly attached to the boron atom was not detected due to quadrupolar relaxation); IR (neat, $\mathrm{cm}^{-1}$ ): 3435, 3027, 2955, 2928, 2870, 2788, 1617, 1494, 1455, 1352, 1287, 1211, 1146, 1107, 1027, 966, 852, 746, 698; HRMS (ESI): $m / z$ calcd for $\mathrm{C}_{31} \mathrm{H}_{47} \mathrm{BNO}_{2}^{+}\left[\mathrm{M}+\mathrm{H}^{+}\right]: 476.3694$. Found: 476.3694.

(Z)-4,4,5,5-tetramethyl-2-(6-phenyldec-5-en-5-yl)-1,3,2-dioxaborolane (4ma)<smiles>BrC(=C(Br)c1ccccc1)c1ccccc1</smiles>

2m $(35.0 \mu \mathrm{L}, 0.200 \mathrm{mmol})$ and $3 \mathbf{a}(68.0 \mu \mathrm{L}, 0.600 \mathrm{mmol})$ were used to give the crude product (r.r. could not be determined due to overlapped impurities). The crude product was purified by silica gel column chromatography (eluent: hexane/AcOEt $=99 / 1)$ to give a mixture of $\mathbf{4 m a}$ and $\mathbf{5 m a}$ as colorless oil $(60.5 \mathrm{mg}, 0.176 \mathrm{mmol}, 88 \%$, r.r. $=13.8: 1)$. Pure $4 \mathrm{ma}$ was 
isolated by PTLC (eluent: hexane/AcOEt $=20 / 1$ ).

4ma: $\mathrm{R}_{\mathrm{f}}=0.34($ hexane/AcOEt $=30 / 1) ;{ }^{1} \mathrm{H} \mathrm{NMR}\left(500 \mathrm{MHz}, \mathrm{CDCl}_{3}\right): \delta 7.29(\mathrm{t}, J=7.4 \mathrm{~Hz}, 2 \mathrm{H}), 7.20(\mathrm{t}, J=7.4 \mathrm{~Hz}, 1 \mathrm{H})$, $7.05(\mathrm{~d}, J=7.4 \mathrm{~Hz}, 2 \mathrm{H}), 2.52(\mathrm{t}, J=7.4 \mathrm{~Hz}, 2 \mathrm{H}), 1.92(\mathrm{t}, J=7.6 \mathrm{~Hz}, 2 \mathrm{H}), 1.32(\mathrm{~s}, 12 \mathrm{H}), 1.25-1.19(\mathrm{~m}, 6 \mathrm{H}), 1.16-1.09$ (m, 2H), $0.82(\mathrm{t}, J=7.0 \mathrm{~Hz}, 3 \mathrm{H}), 0.73(\mathrm{t}, J=7.3 \mathrm{~Hz}, 3 \mathrm{H}) ;{ }^{13} \mathrm{C}$ NMR $\left(125 \mathrm{MHz}, \mathrm{CDCl}_{3}\right): \delta 152.9,143.4,128.0,127.7,126.0$, 83.0, 38.2, 32.6, 31.8, 31.1, 24.8, 22.6, 22.5, 14.0, 13.9 (The carbon directly attached to the boron atom was not detected due to quadrupolar relaxation); IR (neat, $\mathrm{cm}^{-1}$ ): 2956, 2928, 2858, 1616, 1595, 1489, 1352, 1286, 1213, 1137, 1108, 966, 865, 841, 758, 702; HRMS (ESI): $m / z$ calcd for $\mathrm{C}_{22} \mathrm{H}_{35} \mathrm{BNaO}_{2}^{+}\left[\mathrm{M}+\mathrm{Na}^{+}\right]$: 365.2622. Found: 365.2609.

\section{(E)-4,4,5,5-tetramethyl-2-(2-(3-phenylpropyl)hex-1-en-1-yl)-1,3,2-dioxaborolane (4na)}

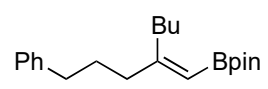

2n $(31.0 \mu \mathrm{L}, 0.200 \mathrm{mmol})$ and $\mathbf{3 a}(68.0 \mu \mathrm{L}, 0.600 \mathrm{mmol})$ were used to give the crude product $($ r.r. $=3.2: 1)$. The crude product was purified by silica gel column chromatography (eluent: hexane/AcOEt $=99 / 1$ ) to give a mixture of $4 \mathbf{m a}$ and $\mathbf{5 m a}$ as colorless oil $(54.9 \mathrm{mg}, 0.167 \mathrm{mmol}, 84 \%$, r.r. $=3.0: 1)$. Pure 4na and 5na was isolated by PTLC (eluent: hexane/AcOEt = 20/1).

4na: $\mathrm{R}_{\mathrm{f}}=0.38$ (twice with hexane/AcOEt $\left.=30 / 1\right) ;{ }^{1} \mathrm{H} \mathrm{NMR}\left(500 \mathrm{MHz}, \mathrm{CDCl}_{3}\right): \delta$ 7.27-7.24 (m, 2H), 7.17-7.15 (m, 3H), $5.13(\mathrm{~s}, 1 \mathrm{H}), 2.60(\mathrm{t}, J=7.7 \mathrm{~Hz}, 2 \mathrm{H}), 2.38(\mathrm{t}, J=7.6 \mathrm{~Hz}, 2 \mathrm{H}), 2.14(\mathrm{t}, J=7.6 \mathrm{~Hz}, 2 \mathrm{H}), 1.80-1.74(\mathrm{~m}, 2 \mathrm{H}), 1.39-1.33(\mathrm{~m}, 2 \mathrm{H})$, 1.32-1.27 (m, 2H), $1.25(\mathrm{~s}, 12 \mathrm{H}), 0.89(\mathrm{t}, J=7.3 \mathrm{~Hz}, 3 \mathrm{H}) ;{ }^{13} \mathrm{C} \mathrm{NMR}\left(125 \mathrm{MHz}, \mathrm{CDCl}_{3}\right): \delta 167.1,142.4,128.4,128.2,125.6$, $82.5,38.5,35.5,34.4,31.7,29.4,24.8,22.6,13.9$ (The carbon directly attached to the boron atom was not detected due to quadrupolar relaxation); IR (neat, $\mathrm{cm}^{-1}$ ): 3405, 2978, 2955, 2930, 2859, 1632, 1491, 1455, 1379, 1324, 1262, 1215, 1142, 1105, 970, 852, 756, 699, 623; HRMS (ESI): $m / z$ calcd for $\mathrm{C}_{21} \mathrm{H}_{33} \mathrm{BNaO}_{2}^{+}\left[\mathrm{M}+\mathrm{Na}^{+}\right]$: 351.2466. Found: 351.2467.

5na: $\mathrm{R}_{\mathrm{f}}=0.57$ (twice with hexane/AcOEt $\left.=30 / 1\right) ;{ }^{1} \mathrm{H} \mathrm{NMR}\left(500 \mathrm{MHz}, \mathrm{CDCl}_{3}\right): \delta 7.25(\mathrm{t}, J=7.4 \mathrm{~Hz}, 2 \mathrm{H}), 7.18-7.14(\mathrm{~m}$, $3 \mathrm{H}), 6.00(\mathrm{t}, J=7.4 \mathrm{~Hz}, 1 \mathrm{H}), 2.58(\mathrm{t}, J=7.9 \mathrm{~Hz}, 2 \mathrm{H}), 2.33-2.28(\mathrm{~m}, 2 \mathrm{H}), 2.14(\mathrm{t}, J=7.6 \mathrm{~Hz}, 2 \mathrm{H}), 1.71-1.65(\mathrm{~m}, 2 \mathrm{H})$, $1.34-1.29(\mathrm{~m}, 4 \mathrm{H}), 1.26(\mathrm{~s}, 12 \mathrm{H}), 0.88(\mathrm{t}, J=7.1 \mathrm{~Hz}, 3 \mathrm{H}) ;{ }^{13} \mathrm{C} \mathrm{NMR}\left(125 \mathrm{MHz}, \mathrm{CDCl}_{3}\right): \delta 146.7,143.0,128.4,128.1,125.4$, $82.7,36.6,35.5,32.2,32.0,30.8,24.8,22.2,13.9$ (The carbon directly attached to the boron atom was not detected due to quadrupolar relaxation); IR (neat, $\mathrm{cm}^{-1}$ ): 3406, 2976, 2956, 2927, 2857, 1629, 1603, 1496, 1455, 1426, 1404, 1389, 1371,

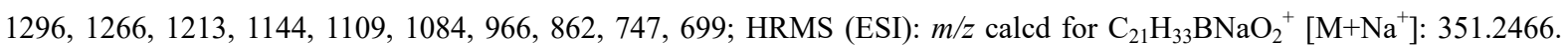
Found: 351.2480 .

\section{(Z)-2-(3-(2-(benzyloxy)ethyl)-6-phenylhex-2-en-2-yl)-4,4,5,5-tetramethyl-1,3,2-dioxaborolane (4ab)}

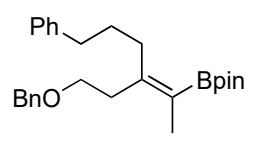

2a $(36.0 \mu \mathrm{L}, 0.200 \mathrm{mmol})$ and $\mathbf{3 b}(96.0 \mu \mathrm{L}, 0.600 \mathrm{mmol})$ were used to give the crude product $($ r.r. $=9.5: 1)$. The crude product was purified by silica gel column chromatography (eluent: hexane/AcOEt $=49 / 1)$ to give an inseparable mixture of 4ab and 5ab as colorless oil (72.3 $\mathrm{mg}, 0.172 \mathrm{mmol}, 86 \%$, r.r. $=10.4: 1)$. 
$\mathrm{R}_{\mathrm{f}}=0.21$ (twice with hexane $\left./ \mathrm{AcOEt}=30 / 1\right) ;{ }^{1} \mathrm{H} \mathrm{NMR}\left(500 \mathrm{MHz}, \mathrm{CDCl}_{3}\right): \delta$ 7.34-7.24 (m, 7H, 4ab $\left.+\mathbf{5 a b}\right), 7.17-7.14(\mathrm{~m}, 3 \mathrm{H}$, 4ab+5ab), 4,50 (s, 2H, 5ab), $4.48(\mathrm{~s}, 2 \mathrm{H}, \mathbf{4 a b}), 3.46(\mathrm{t}, J=7.9 \mathrm{~Hz}, 2 \mathrm{H}, 4 \mathbf{a b}), 3.38(\mathrm{t}, J=7.7 \mathrm{~Hz}, 2 \mathrm{H}, \mathbf{5 a b}), 2.58(\mathrm{t}, J=7.8$ $\mathrm{Hz}, 2 \mathrm{H}, \mathbf{4 a b}+\mathbf{5 a b}), 2.48(\mathrm{t}, J=7.9 \mathrm{~Hz}, 2 \mathrm{H}, \mathbf{4 a b}+\mathbf{5 a b}), 2.36(\mathrm{t}, J=7.9 \mathrm{~Hz}, 2 \mathrm{H}, \mathbf{4 a b}+\mathbf{5 a b}), 1.74-1.66(\mathrm{~m}, 2 \mathrm{H}, \mathbf{4 a b}+\mathbf{5 a b}), 1.69$ (s, 3H, 4ab+5ab), 1.23 (s, 12H, 4ab), 1.19 (s, 12H, 5ab); ${ }^{13} \mathrm{C}$ NMR (125 MHz, $\left.\mathrm{CDCl}_{3}\right): \delta 151.1,142.7,138.4,128.3,128.3$, $128.2,127.6,127.4,125.5,82.8,72.7,68.3,36.4,36.2,33.2,31.7,24.7,24.7,16.3$ (The carbon directly attached to the boron atom was not detected due to quadrupolar relaxation); IR (neat, $\mathrm{cm}^{-1}$ ): 3446, 3025, 2976, 2930, 2857, 1620, 1496, 1454, 1358, 1288, 1214, 1143, 1088, 1028, 965, 856, 750, 698, 670; HRMS (ESI): $m / z$ calcd for $\mathrm{C}_{27} \mathrm{H}_{37} \mathrm{BNaO}_{3}{ }^{+}\left[\mathrm{M}+\mathrm{Na}^{+}\right]$: 443.2728. Found: 443.2736 .

(Z)-2-(3-(2-(benzyloxy)ethyl)octa-2,7-dien-2-yl)-4,4,5,5-tetramethyl-1,3,2-dioxaborolane (4ac)

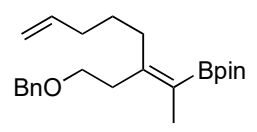

2a $(36.0 \mu \mathrm{L}, 0.200 \mathrm{mmol})$ and $3 \mathbf{c}(74.0 \mu \mathrm{L}, 0.600 \mathrm{mmol})$ were used to give the crude product $(\mathrm{r} . \mathrm{r} .=11.7: 1)$. The crude product was purified by silica gel column chromatography (eluent: hexane/AcOEt $=49 / 1$ ) to give an inseparable mixture of 4ac and 5ac as colorless oil (54.6 $\mathrm{mg}, 0.147 \mathrm{mmol}, 74 \%$, r.r. $=11.2: 1)$.

$\mathrm{R}_{\mathrm{f}}=0.17$ (twice with hexane/AcOEt $\left.=30 / 1\right) ;{ }^{1} \mathrm{H} \mathrm{NMR}\left(500 \mathrm{MHz}, \mathrm{CDCl}_{3}\right): \delta 7.35-7.25(\mathrm{~m}, 5 \mathrm{H}, \mathbf{4 a c}+\mathbf{5 a c}), 5.86-5.78(\mathrm{~m}, 1 \mathrm{H}$, 4ac $+5 \mathbf{a c}), 5.01-4.90(\mathrm{~m}, 2 \mathrm{H}, \mathbf{4 a c}+\mathbf{5 a c}), 4.50(\mathrm{~s}, 2 \mathrm{H}, \mathbf{4 a c}+\mathbf{5 a c}), 3.47(\mathrm{t}, J=7.9 \mathrm{~Hz}, 2 \mathrm{H}, \mathbf{4 a c}), 3.39(\mathrm{t}, J=7.8 \mathrm{~Hz}, 2 \mathrm{H}, \mathbf{5 a c})$, $2.48(\mathrm{t}, J=7.9 \mathrm{~Hz}, 2 \mathrm{H}, \mathbf{4 a c}+\mathbf{5 a c}), 2.30(\mathrm{t}, J=8.0 \mathrm{~Hz}, 2 \mathrm{H}, \mathbf{4 a c}+\mathbf{5 a c}), 2.05-2.00(\mathrm{~m}, 2 \mathrm{H}, \mathbf{4 a c}+\mathbf{5 a c}), 1.74(\mathrm{~s}, 3 \mathrm{H}, \mathbf{5 a c}), 1.69(\mathrm{~s}$, $3 \mathrm{H}, \mathbf{4 a c}), 1.49-1.43(\mathrm{~m}, 2 \mathrm{H}, \mathbf{4 a c}+\mathbf{5 a c}), 1.25(\mathrm{~s}, 12 \mathrm{H}, \mathbf{4 a c}), 1.22(\mathrm{~s}, 12 \mathrm{H}, \mathbf{5 a c}) ;{ }^{13} \mathrm{C} \mathrm{NMR}\left(125 \mathrm{MHz}, \mathrm{CDCl}_{3}\right): \delta 151.2,139.0$, $138.4,128.3,127.6,127.5,114.1,82.8,72.8,68.4,36.1,33.9,33.2,29.3,24.8,24.7,16.3$ (The carbon directly attached to the boron atom was not detected due to quadrupolar relaxation); IR (neat, $\mathrm{cm}^{-1}$ ): 3066, 2977, 2929, 2860, 1620, 1495, 1358, 1293, 1215, 1145, 1089, 1027, 993, 965, 910, 855, 758, 697, 667; HRMS (ESI): $m / z$ calcd for $\mathrm{C}_{23} \mathrm{H}_{35} \mathrm{BNaO}_{3}{ }^{+}\left[\mathrm{M}+\mathrm{Na}^{+}\right]$: 393.2571. Found: 393.2568 .

(Z)-ethyl 5-(2-(benzyloxy)ethyl)-6-(4,4,5,5-tetramethyl-1,3,2-dioxaborolan-2-yl)hept-5-enoate (4ad)

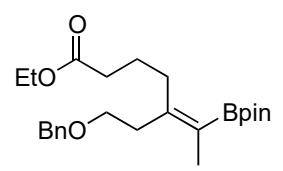

2a $(36.0 \mu \mathrm{L}, 0.200 \mathrm{mmol})$ and $\mathbf{3 d}(89.0 \mu \mathrm{L}, 0.600 \mathrm{mmol})$ were used to give the crude product (r.r. $=9.4: 1)$. The crude product was purified by silica gel column chromatography (eluent: hexane/AcOEt $=49 / 1 \rightarrow 19 / 1$ ) to give an inseparable mixture of $\mathbf{4 a d}$ and $\mathbf{5 a d}$ as colorless oil $(66.9 \mathrm{mg}, 0.161 \mathrm{mmol}, 80 \%$, r.r. $=10.7: 1)$.

$\mathrm{R}_{\mathrm{f}}=0.27$ (twice with hexane/AcOEt $\left.=30 / 1\right) ;{ }^{1} \mathrm{H} \mathrm{NMR}\left(500 \mathrm{MHz}, \mathrm{CDCl}_{3}\right): \delta 7.35-7.24(\mathrm{~m}, 5 \mathrm{H}, \mathbf{4 a d}+\mathbf{5 a d}), 4.50(\mathrm{~s}, 2 \mathrm{H}$, $\mathbf{4 a d}+\mathbf{5 a d}), 4.10(\mathrm{q}, J=7.1 \mathrm{~Hz}, 2 \mathrm{H}, \mathbf{4 a d}+\mathbf{5 a d}), 3.48(\mathrm{t}, J=7.7 \mathrm{~Hz}, 2 \mathrm{H}, \mathbf{4 a d}), 3.38(\mathrm{t}, J=7.7 \mathrm{~Hz}, 2 \mathrm{H}, \mathbf{5 a d}), 2.49(\mathrm{t}, J=7.7 \mathrm{~Hz}$, $2 \mathrm{H}, \mathbf{4 a d}+\mathbf{5 a d}), 2.26(\mathrm{t}, J=7.7 \mathrm{~Hz}, 2 \mathrm{H}, \mathbf{4 a d}+\mathbf{5 a d}), 1.75-1.66(\mathrm{~m}, 2 \mathrm{H}, \mathbf{4 a d}+\mathbf{5 a d}), 1.69(\mathrm{~s}, 3 \mathrm{H}, \mathbf{4 a d}+\mathbf{5 a d}), 1.26-1.21(\mathrm{~m}, 3 \mathrm{H}$, 4ad+5ad), $1.25(\mathrm{~s}, 12 \mathrm{H}, \mathbf{4 a d}+\mathbf{5 a d}) ;{ }^{13} \mathrm{C} \mathrm{NMR}\left(125 \mathrm{MHz}, \mathrm{CDCl}_{3}\right): \delta 173.8,150.7,138.4,128.3,127.5,127.4,82.8,72.8$, 
$68.3,60.1,35.5,34.2,33.1,25.0,24.8,24.7,16.4,14.2$ (The carbon directly attached to the boron atom was not detected due to quadrupolar relaxation); IR (neat, $\mathrm{cm}^{-1}$ ): 3446, 2977, 2933, 2863, 1734, 1621, 1455, 1357, 1294, 1247, 1213, 1144, 1090, 1028, 965, 856, 737, 697, 671; HRMS (ESI): $m / z$ calcd for $\mathrm{C}_{24} \mathrm{H}_{37} \mathrm{BNaO}_{5}^{+}\left[\mathrm{M}+\mathrm{Na}^{+}\right]:$439.2626. Found: 439.2631.

(Z)-2-(5-(benzyloxy)-3-(cyclopropylmethyl)pent-2-en-2-yl)-4,4,5,5-tetramethyl-1,3,2-dioxaborolane (4ae)<smiles>C/C(Cc1ccccc1)=C(\CCOCc1ccccc1)CC1CC1</smiles>

2a $(36.0 \mu \mathrm{L}, 0.200 \mathrm{mmol})$ and $3 \mathrm{e}(63.0 \mu \mathrm{L}, 0.600 \mathrm{mmol})$ were used to give the crude product $(\mathrm{r} . \mathrm{r} .=12.1: 1)$. The crude product was purified by silica gel column chromatography (eluent: hexane/AcOEt $=49 / 1$ ) to give an inseparable mixture of 4ae and 5ae as colorless oil (56.0 $\mathrm{mg}, 0.172 \mathrm{mmol}, 86 \%$, r.r. $=11.2: 1)$.

$\mathrm{R}_{\mathrm{f}}=0.13$ (twice with hexane/AcOEt $\left.=30 / 1\right) ;{ }^{1} \mathrm{H}$ NMR (500 MHz, $\left.\mathrm{CDCl}_{3}\right): \delta$ 7.36-7.25 (m, 5H, 4ae+5ae), $4.51(\mathrm{~s}, 2 \mathrm{H}$, 4ae+5ae), 3.51 (t, 2H, $J=8.0 \mathrm{~Hz}, 4 \mathbf{a e}), 3.40(\mathrm{t}, J=7.7 \mathrm{~Hz}, 2 \mathrm{H}, 5 \mathbf{a e}), 2.59$ (t, $J=8.0 \mathrm{~Hz}, 2 \mathrm{H}, 4 \mathbf{a e}), 2.50(\mathrm{t}, J=7.7 \mathrm{~Hz}, 2 \mathrm{H}$, 5ae), $2.25(\mathrm{~d}, J=6.5 \mathrm{~Hz}, 2 \mathrm{H}, \mathbf{4 a e}+5 \mathbf{a e}), 1.82(\mathrm{~s}, 2 \mathrm{H}, \mathbf{5 a e}), 1.71(\mathrm{~s}, 2 \mathrm{H}, \mathbf{4 a e}), 1.24(\mathrm{~s}, 12 \mathrm{H}, 4 \mathbf{a e}), 1.21$ (s, 12H, 5ae), 0.82-0.75 (m, 1H, 4ae+5ae), 0.40-0.35 (m, 2H, 4ae+5ae), 0.14-0.11 (m, 2H, 4ae+5ae); $\left.{ }^{13} \mathrm{C} \mathrm{NMR} \mathrm{(125} \mathrm{MHz,} \mathrm{CDCl} 3\right): \delta 138.5,128.3$, $127.6,127.4,82.8,72.7,68.3,40.5,33.1,24.7,16.3,10.8,4.4$ (The carbon directly attached to the boron atom was not detected due to quadrupolar relaxation); IR (neat, $\mathrm{cm}^{-1}$ ): 3396, 3073, 2977, 2929, 2858, 1621, 1455, 1355, 1290, 1214, 1147, 1087, 1016, 966, 854, 736, 697; HRMS (ESI): $m / z$ calcd for $\mathrm{C}_{22} \mathrm{H}_{33} \mathrm{BNaO}_{3}{ }^{+}\left[\mathrm{M}+\mathrm{Na}^{+}\right]: 379.2415$. Found: 379.2422.

(Z)-2-(5-(benzyloxy)-3-methylpent-2-en-2-yl)-4,4,5,5-tetramethyl-1,3,2-dioxaborolane (4af)<smiles>C/C(CCOCc1ccccc1)=C(\C)CCOCc1ccccc1</smiles>

2a $(36.0 \mu \mathrm{L}, 0.200 \mathrm{mmol})$, 3f $(2.0 \mathrm{M}$ in DMF, $0.300 \mathrm{~mL}, 0.600 \mathrm{mmol})$ and $\mathrm{DMF}(0.70 \mathrm{~mL})$ were used. 3f was added dropwise over $1 \mathrm{~h}$ at ambient temperature. The resulting mixture was stirred for another $3 \mathrm{~h}$ to give the crude product (r.r. $=$ 14.1 : 1). The crude product was purified by silica gel column chromatography (eluent: hexane/AcOEt $=49 / 1)$ to give an inseparable mixture of $\mathbf{4 a f}$ and 5 af as sticky white solids $(52.4 \mathrm{mg}, 0.166 \mathrm{mmol}, 83 \%$, r.r. $=14.5: 1)$.

$\mathrm{R}_{\mathrm{f}}=0.35$ (twice with hexane/AcOEt $\left.=30 / 1\right) ;{ }^{1} \mathrm{H}$ NMR $\left(500 \mathrm{MHz}, \mathrm{CDCl}_{3}\right): \delta 7.35-7.25(\mathrm{~m}, 5 \mathrm{H}, 4 \mathbf{a f}+\mathbf{5 a f}), 4.51(\mathrm{~s}, 2 \mathrm{H}$, 4af $+5 \mathbf{a f}), 3.51(\mathrm{t}, J=7.6 \mathrm{~Hz}, 2 \mathrm{H}, \mathbf{4 a f}), 3.39$ (t, $J=7.8 \mathrm{~Hz}, 2 \mathrm{H}, \mathbf{5 a f}), 2.48(\mathrm{t}, J=7.6 \mathrm{~Hz}, 2 \mathrm{H}, 4 \mathbf{a f}+\mathbf{5 a f}), 1.97$ (d, $J=1.5 \mathrm{~Hz}$ $3 \mathrm{H}, \mathbf{4 a f}+5 \mathbf{a f}), 1.75$ (s, 3H, 5af), $1.69(\mathrm{~s}, 3 \mathrm{H}, \mathbf{4 a f}), 1.26$ (s, 12H, 4af), $1.23(\mathrm{~s}, 12 \mathrm{H}, 5 \mathbf{5 a f}) ;{ }^{13} \mathrm{C} \mathrm{NMR}\left(125 \mathrm{MHz}, \mathrm{CDCl}_{3}\right): \delta$ $147.4,138.5,128.3,127.6,127.4,82.8,72.8,68.1,35.3,24.8,24.7,22.8,16.2$ (The carbon directly attached to the boron atom was not detected due to quadrupolar relaxation); IR (neat, $\mathrm{cm}^{-1}$ ): 3445, 2977, 2929, 2863, 1733, 1626, 1455, 1358, 1292, 1214, 1147, 1088, 965, 854, 735, 697, 685, 635; HRMS (ESI): $m / z$ calcd for $\mathrm{C}_{19} \mathrm{H}_{29} \mathrm{BNaO}_{3}{ }^{+}\left[\mathrm{M}+\mathrm{Na}^{+}\right]: 339.2102$. Found: 339.2115. 
<smiles>C/C(Cc1ccccc1)=C(\CCOCc1ccccc1)c1ccccc1</smiles>

2a $(36.0 \mu \mathrm{L}, 0.200 \mathrm{mmol})$ and $3 \mathrm{~g}(71.0 \mu \mathrm{L}, 0.600 \mathrm{mmol})$ were used to give the crude product (r.r. $=12.8: 1)$. The crude product was purified by silica gel column chromatography (eluent: hexane/AcOEt $=49 / 1 \rightarrow 19 / 1$ ) to give an inseparable mixture of $\mathbf{4 a g}$ and $\mathbf{5 a g}$ as colorless oil $(56.7 \mathrm{mg}, 0.144 \mathrm{mmol}, 72 \%$, r.r. $=12.6: 1)$.

$\mathrm{R}_{\mathrm{f}}=0.15$ (twice with hexane/AcOEt $\left.=30 / 1\right) ;{ }^{1} \mathrm{H} \mathrm{NMR}\left(500 \mathrm{MHz}, \mathrm{CDCl}_{3}\right): \delta$ 7.34-7.14 (m, $\left.10 \mathrm{H}, \mathbf{4 a g}+\mathbf{5 a g}\right), 4.51(\mathrm{~s}, 2 \mathrm{H}$, 5ag), 4.41 (s, 2H, 4ag), 3.72 (s, 2H, 4ag), 3.70 (s, 2H, 5ag), 3.45 (t, J= 7.4 Hz, 2H, 5ag), 3.33 (t, J= 7.7 Hz, 2H, 4ag), 2.54 (t, $J=7.4 \mathrm{~Hz}, 2 \mathrm{H}, \mathbf{5 a g}), 2.38(\mathrm{t}, J=7.7 \mathrm{~Hz}, 2 \mathrm{H}, \mathbf{4 a g}), 1.76(\mathrm{~s}, 3 \mathrm{H}, \mathbf{4 a g}), 1.63$ (s, 3H, 5ag), 1.26 (s, 12H, 4ag), $1.23(\mathrm{~s}, 12 \mathrm{H}$, 5ag); ${ }^{13} \mathrm{C}$ NMR (125 MHz, $\left.\mathrm{CDCl}_{3}\right): \delta 149.5,140.7,138.5,129.0,128.3,128.1,127.5,127.4,125.7,83.0,72.6,68.2,42.3$, 32.5, 24.7, 16.5 (The carbon directly attached to the boron atom was not detected due to quadrupolar relaxation); IR (neat, $\left.\mathrm{cm}^{-1}\right): 3421,3024,2976,2929,2858,1623,1455,1353,1301,1214,1143,1081,966,854,754,699 ;$ HRMS (ESI): $m / z$ calcd for $\mathrm{C}_{25} \mathrm{H}_{33} \mathrm{BNaO}_{3}^{+}\left[\mathrm{M}+\mathrm{Na}^{+}\right]$: 415.2415. Found: 415.2404.

(E)-ethyl 3-(2-(benzyloxy)ethyl)-4-(4,4,5,5-tetramethyl-1,3,2-dioxaborolan-2-yl)pent-3-enoate (4ah)<smiles>CCOC(=O)C/C(CCCOCc1ccccc1)=C(\C)Cc1ccccc1</smiles>

2a $(36.0 \mu \mathrm{L}, 0.200 \mathrm{mmol})$ and $\mathbf{3 h}(66.0 \mu \mathrm{L}, 0.600 \mathrm{mmol})$ were used to give the crude product (r.r. could not be determined due to overlapped impurities). The crude product was purified by silica gel column chromatography (eluent: hexane/AcOEt $=19 / 1 \rightarrow 9 / 1)$ and GPC to give an inseparable mixture of $\mathbf{4 a h}$ and $\mathbf{5 a h}$ as colorless oil $(33.4 \mathrm{mg}, 0.0860 \mathrm{mmol}, 43 \%$, r.r. $=$ $13.0: 1)$.

$\mathrm{R}_{\mathrm{f}}=0.22$ (twice with hexane/AcOEt $\left.=30 / 1\right) ;{ }^{1} \mathrm{H}$ NMR (500 MHz, $\left.\mathrm{CDCl}_{3}\right): \delta$ 7.35-7.24 (m, 5H, 4ah $\left.+\mathbf{5 a h}\right), 4.51(\mathrm{~s}, 2 \mathrm{H}, \mathbf{5} \mathbf{a h})$, 4.49 (s, 2H, 4ah), 4.08 (q, $J=7.1 \mathrm{~Hz}, 2 \mathrm{H}, \mathbf{4 a h}+\mathbf{5 a h}), 3.51(\mathrm{~s}, 2 \mathrm{H}, \mathbf{4} \mathbf{a h}+\mathbf{5} \mathbf{a h}), 3.51(\mathrm{t}, J=7.3 \mathrm{~Hz}, 2 \mathrm{H}, \mathbf{4 a h}), 3.42(\mathrm{t}, J=7.6$ $\mathrm{Hz}, 2 \mathrm{H}, \mathbf{5 a h}), 2.56$ (t, $J=7.3 \mathrm{~Hz}, 2 \mathrm{H}, \mathbf{4 a h}+\mathbf{5} \mathbf{a h}), 1.83$ (s, 3H, 5ah), 1.76 (s, 3H, 4ah), 1.26-1.21 (m, 3H, 4ah+5ah), 1.24 (s, 12H, 4ah+5ah); ${ }^{13} \mathrm{C}$ NMR (125 MHz, $\left.\mathrm{CDCl}_{3}\right): \delta$ 172.3, 144.0, 138.4, 128.3, 127.5, 127.4, 83.0, 72.8, 68.1, 60.2, 41.1, 34.1, 24.7, 16.5, 14.2 (The carbon directly attached to the boron atom was not detected due to quadrupolar relaxation); IR (neat, $\left.\mathrm{cm}^{-1}\right): 3450,2979,2933,2858,1731,1628,1455,1359,1301,1215,1143,1094,1030,966,853,755,698,668$; HRMS (ESI): $m / z$ calcd for $\mathrm{C}_{22} \mathrm{H}_{33} \mathrm{BNaO}_{5}^{+}\left[\mathrm{M}+\mathrm{Na}^{+}\right]:$411.2313. Found: 411.2308.

(Z)-2-(3-(2-(benzyloxy)ethyl)-5-methylhex-2-en-2-yl)-4,4,5,5-tetramethyl-1,3,2-dioxaborolane (4ai)<smiles>CC(Cc1ccccc1)=C(CCOCc1ccccc1)CC(C)C</smiles>

2a $(36.0 \mu \mathrm{L}, 0.200 \mathrm{mmol})$ and $3 \mathbf{i}(69.0 \mu \mathrm{L}, 0.600 \mathrm{mmol})$ were used to give the crude product $($ r.r. $=5.4: 1)$. The crude 
product was purified by silica gel column chromatography (eluent: hexane/AcOEt $=49 / 1$ ) to give an inseparable mixture of 4ai and 5ai as colorless oil $(21.7 \mathrm{mg}, 0.0606 \mathrm{mmol}, 30 \%$, r.r. $=4.8: 1)$.

$\mathrm{R}_{\mathrm{f}}=0.27$ (twice with hexane/AcOEt $\left.\left.=30 / 1\right) ;{ }^{1} \mathrm{H} \mathrm{NMR} \mathrm{(500} \mathrm{MHz,} \mathrm{CDCl}_{3}\right): \delta$ 7.35-7.25 (m, 5H, 4ai +5ai), 4.51 (s, 2H, 5ai), 4.50 (s, 2H, 4ai), 3.46 (t, $J=8.0 \mathrm{~Hz}, 2 \mathrm{H}, 4 \mathbf{a i}), 3.40$ (t, $J=7.8 \mathrm{~Hz}, 2 \mathrm{H}, \mathbf{5 a i}), 2.47$ (t, $J=8.0 \mathrm{~Hz}, 2 \mathrm{H}, \mathbf{4 a i}+\mathbf{5 a i}), 2.24$ (d, $J=7.3$ $\mathrm{Hz}, 2 \mathrm{H}, \mathbf{5 a i}), 2.21$ (d, J=7.2 Hz, 2H, 4ai), 1.73-1.69 (m, 1H, 4ai+5ai), $1.72(\mathrm{~s}, 3 \mathrm{H}, \mathbf{5 a i}), 1.71$ (s, 3H, 4ai), 1.25 (s, 12H, 4ai), 1.22 (s, 12H, 5ai), 0.84 (d, $J=6.5 \mathrm{~Hz}, 6 \mathrm{H}, 4 \mathbf{a i}), 0.83$ (d, $J=6.5 \mathrm{~Hz}, 6 \mathrm{H}, \mathbf{5 a i}) ;{ }^{13} \mathrm{C} \mathrm{NMR}\left(125 \mathrm{MHz}, \mathrm{CDCl}_{3}\right): \delta 149.9,138.5$, $128.3,128.2,127.6,127.5,127.4,82.7,72.7,68.3,45.0,33.2,27.9,24.8,24.7,22.5,16.4$ (The carbon directly attached to the boron atom was not detected due to quadrupolar relaxation); IR (neat, $\mathrm{cm}^{-1}$ ): 3420, 2972, 2955, 2929, 2866, 1619, 1455, 1356, 1294, 1215, 1147, 1130, 1087, 966, 855, 755, 697, 668; HRMS (ESI): $m / z$ calcd for $\mathrm{C}_{22} \mathrm{H}_{35} \mathrm{BNaO}_{3}^{+}\left[\mathrm{M}+\mathrm{Na}^{+}\right]$: 381.2571. Found: 381.2565 .

\section{Applications}

\subsection{Suzuki-Miyaura Coupling with PhI}

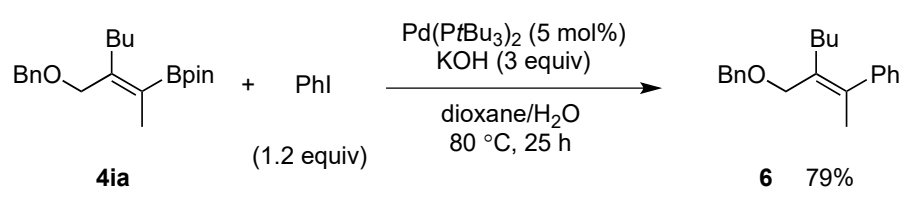

(E)-3-(benzyloxy)methyl-2-phenyl-2-heptene (6)

To a dried screw cap tube were added $4 \mathbf{i a}(27.6 \mathrm{mg}, 0.0802 \mathrm{mmol})$, iodobenzene $(10.7 \mu \mathrm{L}, 0.0960 \mathrm{mmol}),\left(t \mathrm{Bu}_{3} \mathrm{P}\right)_{2} \mathrm{Pd}(2.0$ $\mathrm{mg}, 0.0040 \mathrm{mmol}), \mathrm{KOH}(13.5 \mathrm{mg}, 0.240 \mathrm{mmol}), 1,4-$ dioxane $(0.16 \mathrm{~mL})$ and water $(0.040 \mathrm{~mL})$. After the tube was sealed with a cap, the resulting mixture was stirred at $80{ }^{\circ} \mathrm{C}$ for $25 \mathrm{~h}$. The reaction was quenched with water, and extracted three times with hexane/AcOEt $=4 / 1$. After filtration over $\mathrm{Na}_{2} \mathrm{SO}_{4}$ and evaporation, the residue was purified by silica gel column chromatography (eluent: hexane/AcOEt $=99 / 1)$ to give 6 as NMR-pure brown oil $(18.6 \mathrm{mg}, 0.0632 \mathrm{mmol}, 79 \%)$. Colorless oil 6 was obtained after GPC.

$\mathrm{R}_{\mathrm{f}}=0.30$ (twice with hexane/AcOEt $\left.=30 / 1\right) ;{ }^{1} \mathrm{H}$ NMR $\left(500 \mathrm{MHz}, \mathrm{CDCl}_{3}\right): \delta$ 7.40-7.34 (m, 4H), 7.32- 7.28 (m, 3H), 7.21 (t, $J=7.1 \mathrm{~Hz}, 1 \mathrm{H}), 7.11(\mathrm{~d}, J=7.1 \mathrm{~Hz}, 2 \mathrm{H}), 4.56(\mathrm{~s}, 2 \mathrm{H}), 4.15(\mathrm{~s}, 2 \mathrm{H}), 1.98(\mathrm{t}, J=7.7 \mathrm{~Hz}, 2 \mathrm{H}), 1.98(\mathrm{~s}, 3 \mathrm{H}), 1.32-1.25(\mathrm{~m}, 2 \mathrm{H})$, 1.16-1.08 (m, 2H), $0.74(\mathrm{t}, J=7.3 \mathrm{~Hz}, 3 \mathrm{H}) ;{ }^{13} \mathrm{C} \mathrm{NMR}\left(125 \mathrm{MHz}, \mathrm{CDCl}_{3}\right): \delta 144.6,138.6,136.3,132.5,128.3,128.0,127.8$, 127.8, 127.5, 126.1 , 72.2, 68.3, 31.4, 30.9, 22.6, 21.1, 13.8; IR (neat, $\mathrm{cm}^{-1}$ ): 3420, 2950, 2925, 2858, 2358, 1646, 1455, 1361, 1214, 1087, 1071, 1026, 764, 700, 635; HRMS (ESI): $m / z$ calcd for $\mathrm{C}_{21} \mathrm{H}_{26} \mathrm{NaO}^{+}\left[\mathrm{M}+\mathrm{Na}^{+}\right]$: 317.1876. Found: 317.1878.

\subsection{Suzuki-Miyaura Coupling with $\mathrm{C}_{12} \mathrm{H}_{25} \mathrm{Br}$}

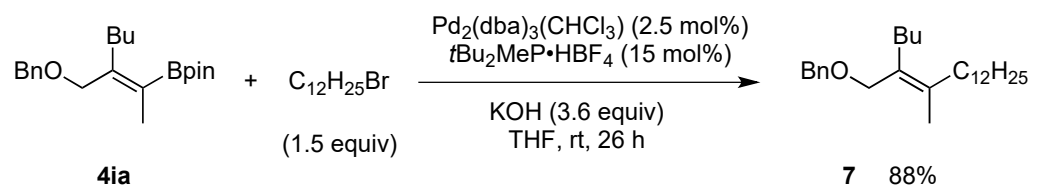

(E)-5-(benzyloxy)methyl-6-methyl-5-octadecene (7)

To a dried screw cap tube were added 4ia $(26.2 \mathrm{mg}, 0.0760 \mathrm{mmol}), \mathrm{Pd}_{2}\left(\mathrm{dba}_{3} \cdot \mathrm{CHCl}_{3}(2.0 \mathrm{mg}, 0.0019 \mathrm{mmol})\right.$, 
$t \mathrm{Bu}_{2} \mathrm{MeP} \mathrm{HBF}_{4}(2.8 \mathrm{mg}, 0.011 \mathrm{mmol}), \mathrm{KOH}(15.4 \mathrm{mg}, 0.274 \mathrm{mmol})$ and THF $(0.19 \mathrm{~mL})$. The resulting mixture was stirred for $20 \mathrm{~min}$, before 1-bromododecane $(27.0 \mu \mathrm{L}, 0.114 \mathrm{mmol})$ was added. After the tube was sealed with a cap, the resulting mixture was stirred at ambient temperature for $26 \mathrm{~h}$. The reaction mixture was filtrated through a short pad of silica gel (eluted with hexane/AcOEt $=10 / 1)$. After evaporation, the residue was purified by silica gel column chromatography (eluent: hexane/AcOEt $=99 / 1 \rightarrow 49 / 1)$ to give 7 as yellow oil $(26.0 \mathrm{mg}, 0.0672 \mathrm{mmol}, 88 \%)$.

$\mathrm{R}_{\mathrm{f}}=0.44$ (twice with hexane/AcOEt $\left.=30 / 1\right) ;{ }^{1} \mathrm{H}$ NMR $\left(500 \mathrm{MHz}, \mathrm{CDCl}_{3}\right): \delta$ 7.37-7.31 (m, 4H), 7.29-7.25 (m, $\left.1 \mathrm{H}\right), 4.45(\mathrm{~s}$, 2H), $3.98(\mathrm{~s}, 2 \mathrm{H}), 2.11$ (t, $J=7.7 \mathrm{~Hz}, 2 \mathrm{H}), 2.03$ (t, $J=7.7 \mathrm{~Hz}, 2 \mathrm{H}), 1.67(\mathrm{~s}, 3 \mathrm{H}), 1.55-1.25(\mathrm{~m}, 24 \mathrm{H}), 0.89$ (t, $J=6.8 \mathrm{~Hz}, 3 \mathrm{H})$, $0.87(\mathrm{t}, J=6.8 \mathrm{~Hz}, 3 \mathrm{H}) ;{ }^{13} \mathrm{C} \mathrm{NMR}\left(125 \mathrm{MHz}, \mathrm{CDCl}_{3}\right): \delta 138.8,135.2,129.8,128.2,127.8,127.4,71.7,69.1,34.4,31.9$, $31.3,30.3,29.8,29.7,29.6,29.6,29.6,29.6,29.3,28.3,23.0,22.7,18.0,14.1,14.0$; IR (neat, $\mathrm{cm}^{-1}$ ): 3406, 2955, 2924, 2854, $1495,1455,1376,1355,1214,1087,1069,1027,938,732,696$; HRMS (ESI): $m / z$ calcd for $\mathrm{C}_{27} \mathrm{H}_{46} \mathrm{ONa}^{+}\left[\mathrm{M}^{+} \mathrm{Na}^{+}\right]$: 409.3441. Found: 409.3449 .

\section{References}

1. Hasse, M. J.; Butts, C. P.; Willis, C. L.; Aggarwal, V. K. Angew. Chem. Int. Ed. 2012, 51, 12444.

2. Alonso, F.; Osante, I.; Yus, M. Tetrahedron 2007, 63, 93.

3. Guy, A.; Oger, C.; Heppekausen, J.; Signorini, C.; Felice, C. D.; Fürstner, A.; Durand, T.; Galano, J.-M. Chem. Eur. J. 2014, 20, 6374 .

4. Hosoya, T.; Wakao, M.; Kondo, Y.; Doi, H.; Suzuki, M. Org. Biomol. Chem. 2004, 2, 24.

5. Fischer, M.; Schemölzer, C.; Nowikow, C.; Schmid, W. Eur, J. Org. Chem. 2011, 1645.

6. Kabalka, G. W.; Venkataiah, B.; Dong, G. Tetrahedron Lett. 2004, 45, 729.

7. Lin, Y. A.; Chalker, J. M.; Floyd, N.; Bernardes, G. J. L.; Davis, B. G.; J. Am. Chem. Soc. 2008, 130, 9642.

8. Kucharski, T. J.; Ferralis, N.; Kolpak, A. M.; Zheng, J. O.; Nocera, D. G.; Grossman, J. C. Nature Chem. 2014, 6, 441.

9. Tennyson, A. G.; Lynch, V. M.; Bielawski, C. W. J. Am. Chem. Soc. 2010, 132, 9420. 


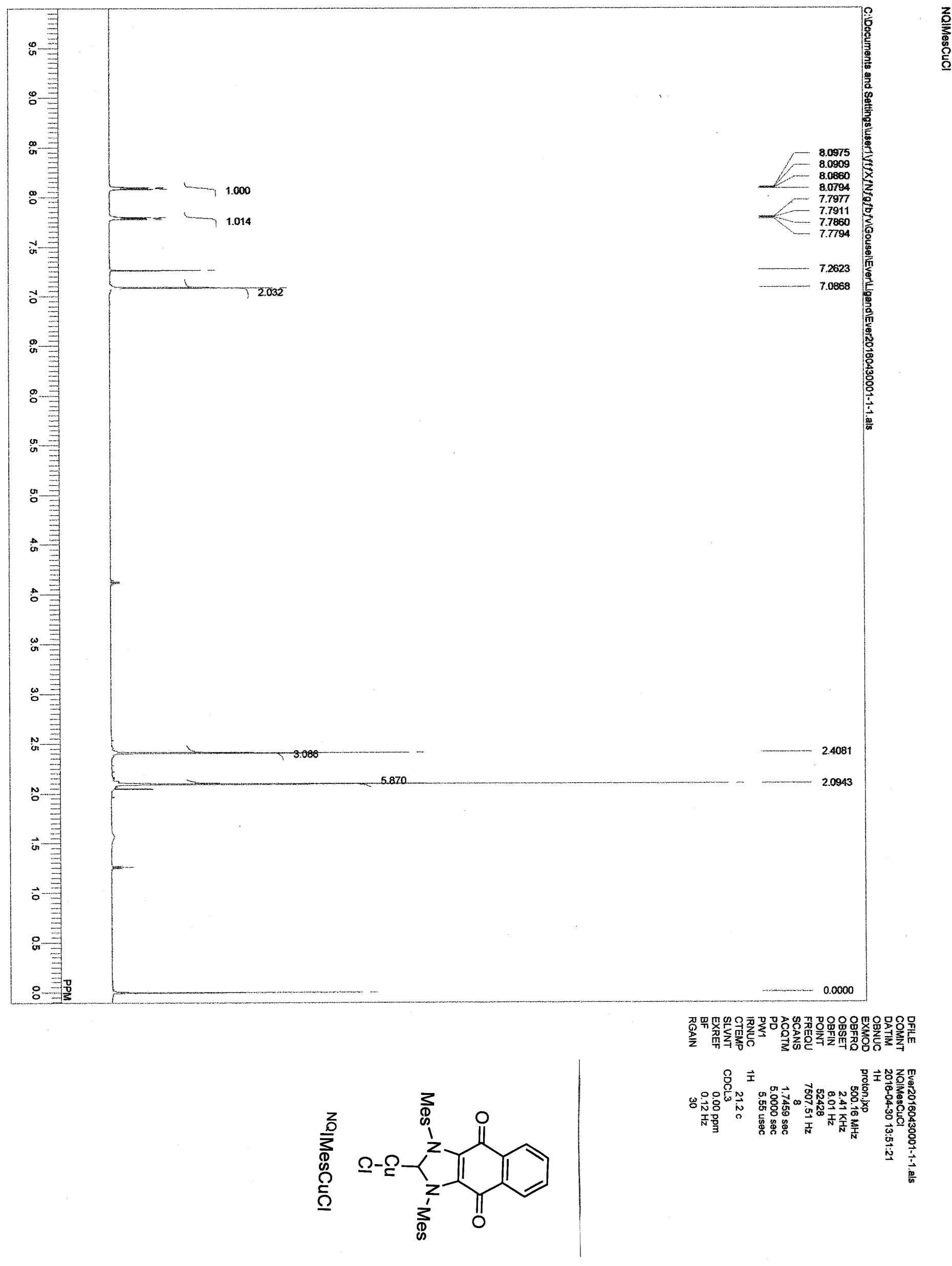




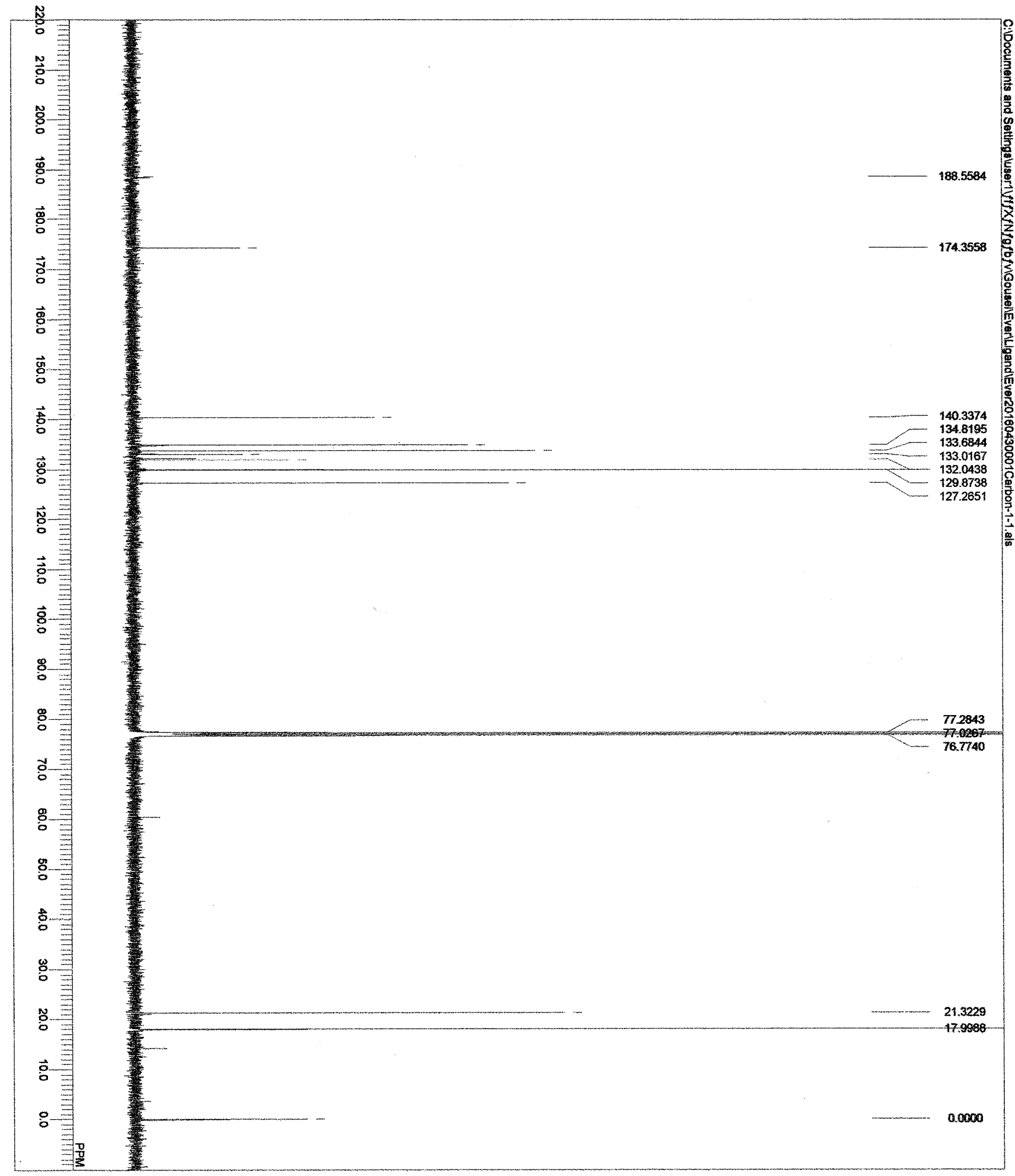

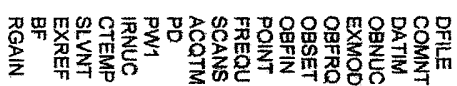

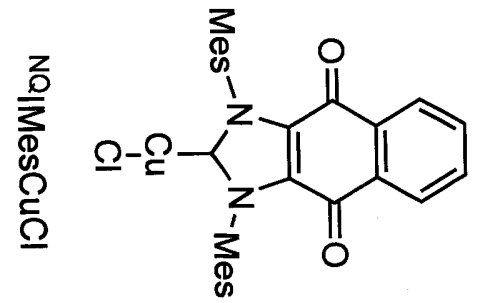

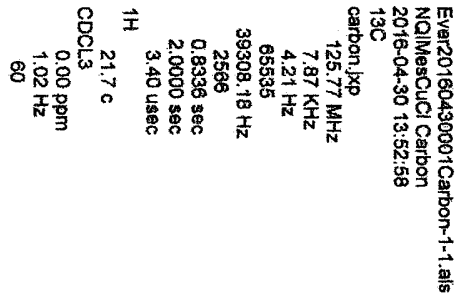



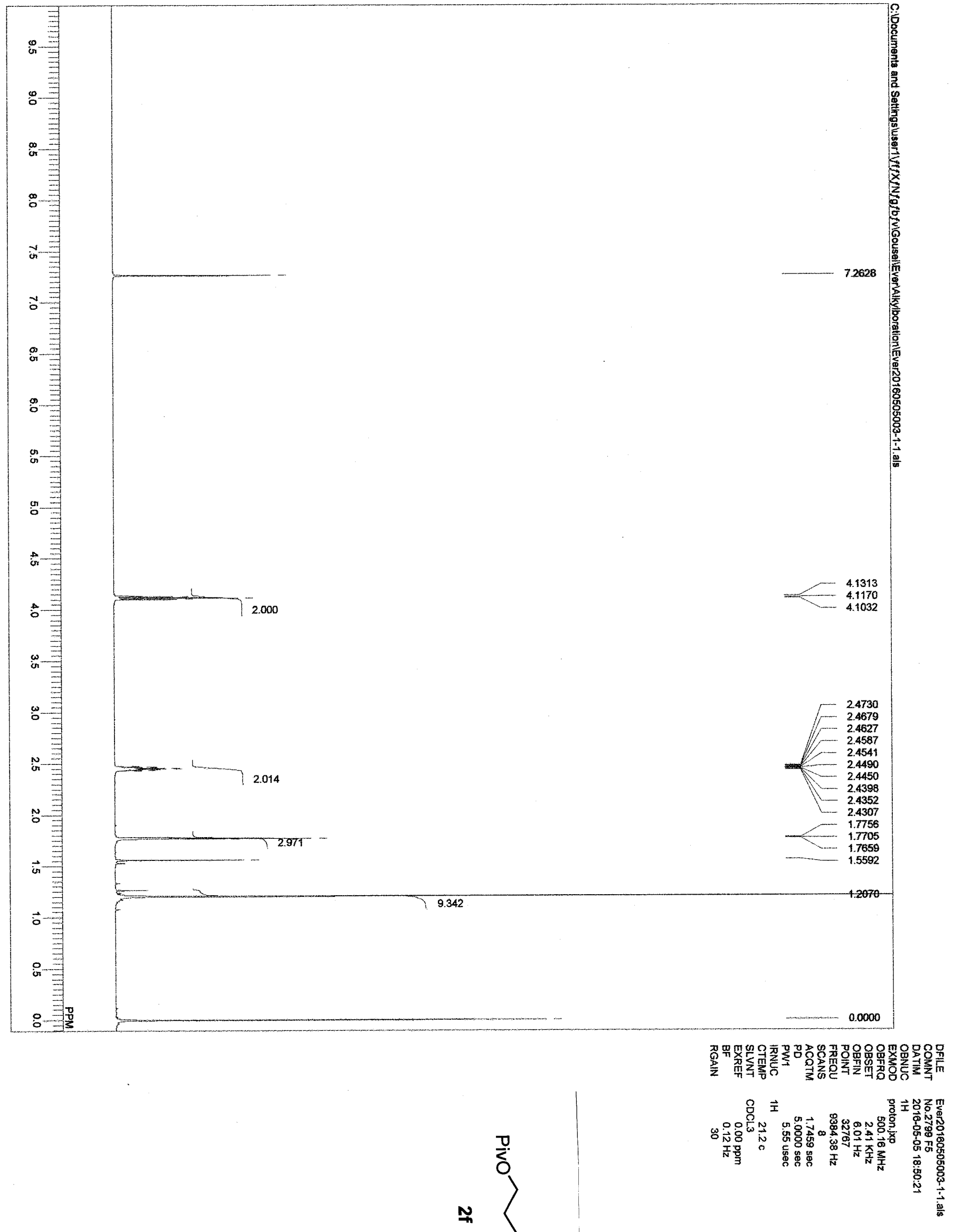


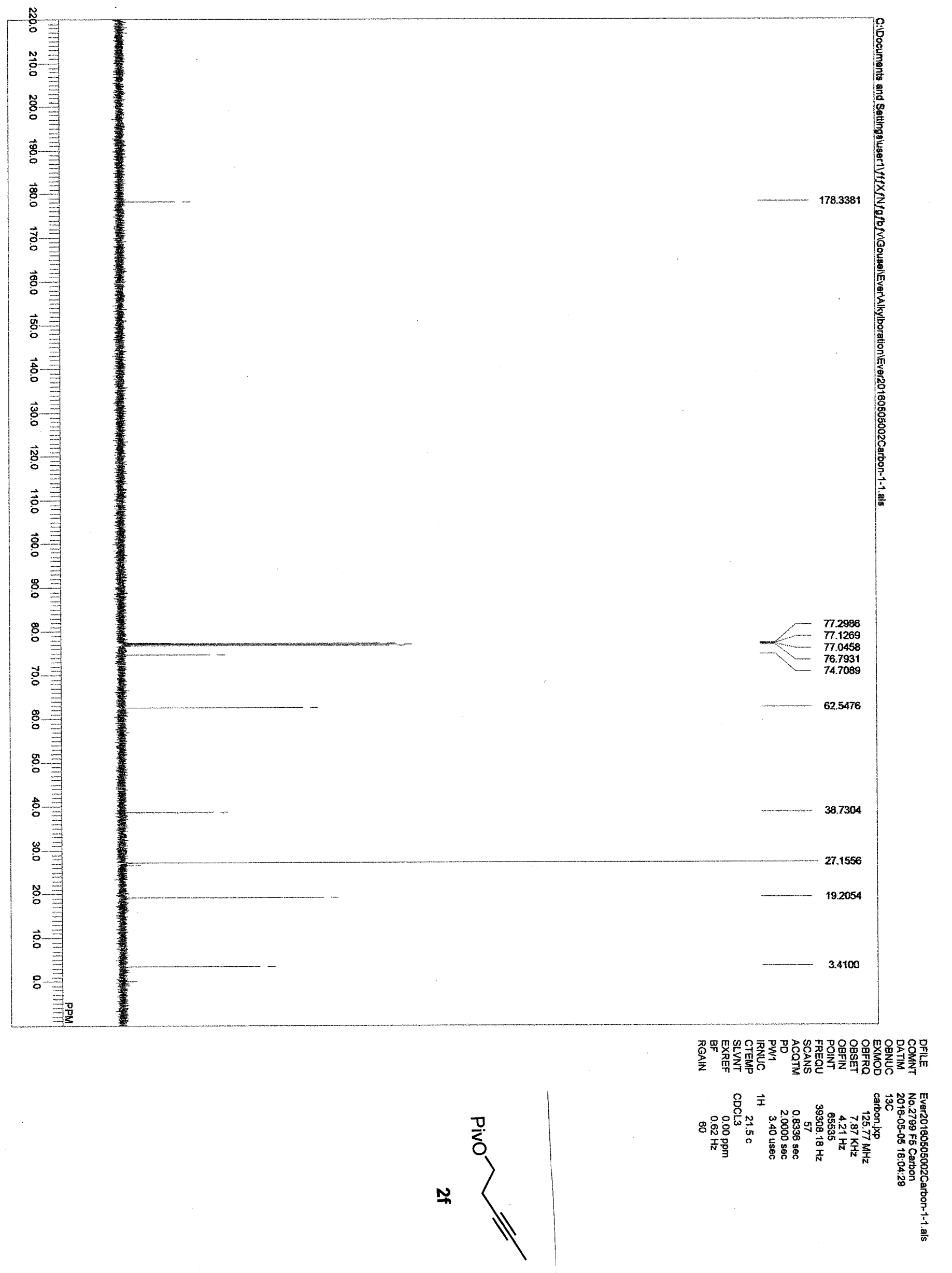




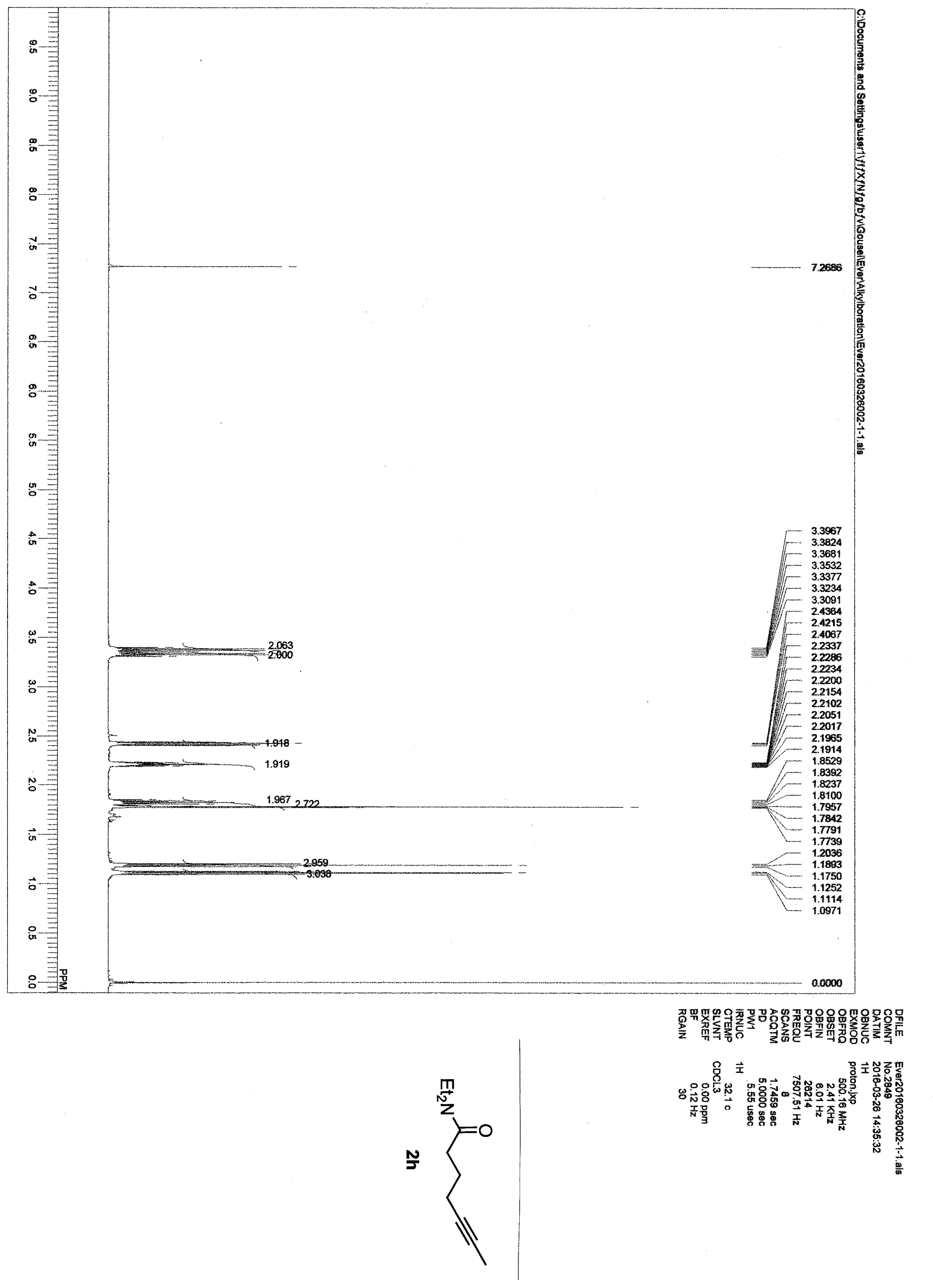




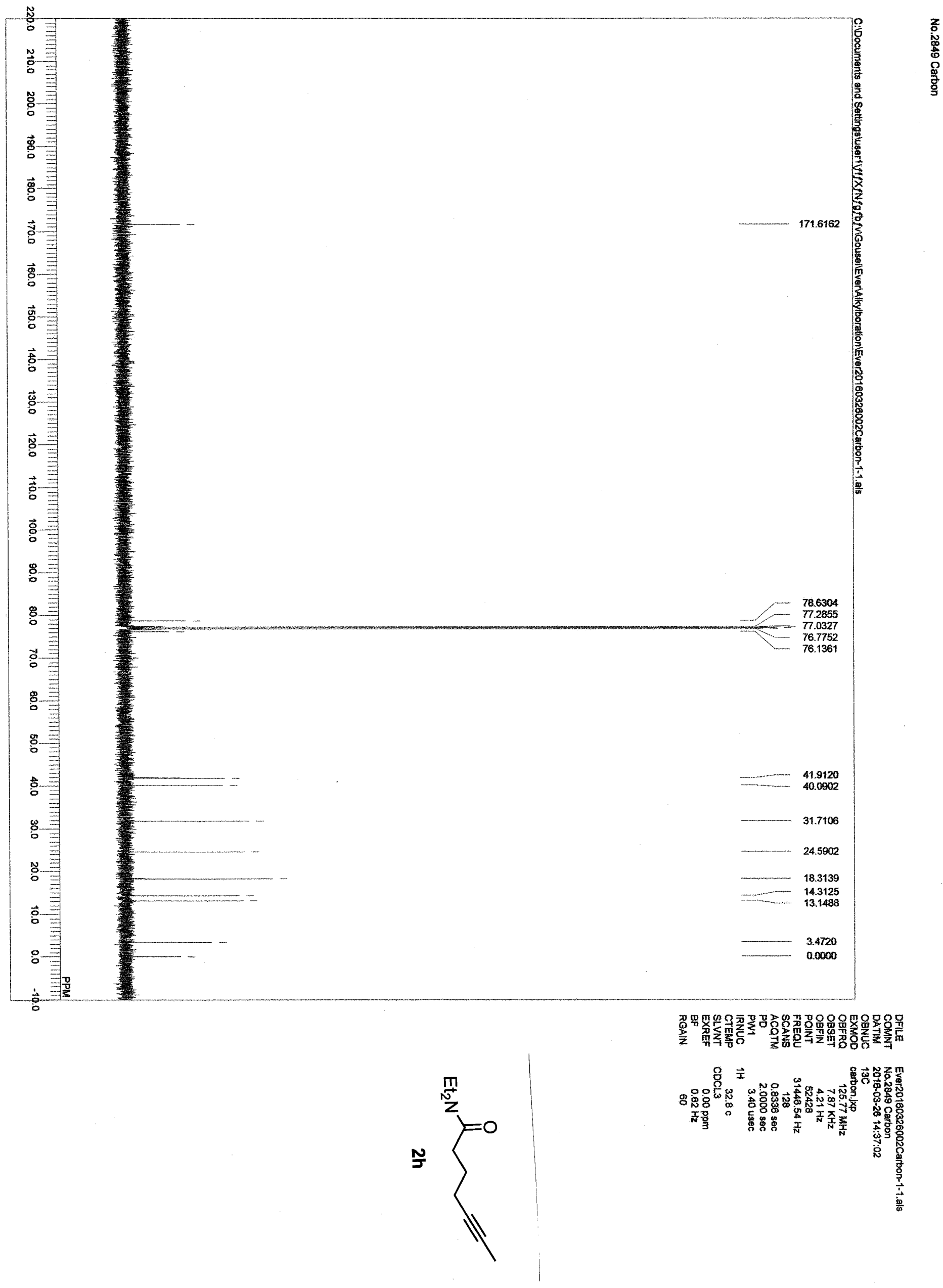




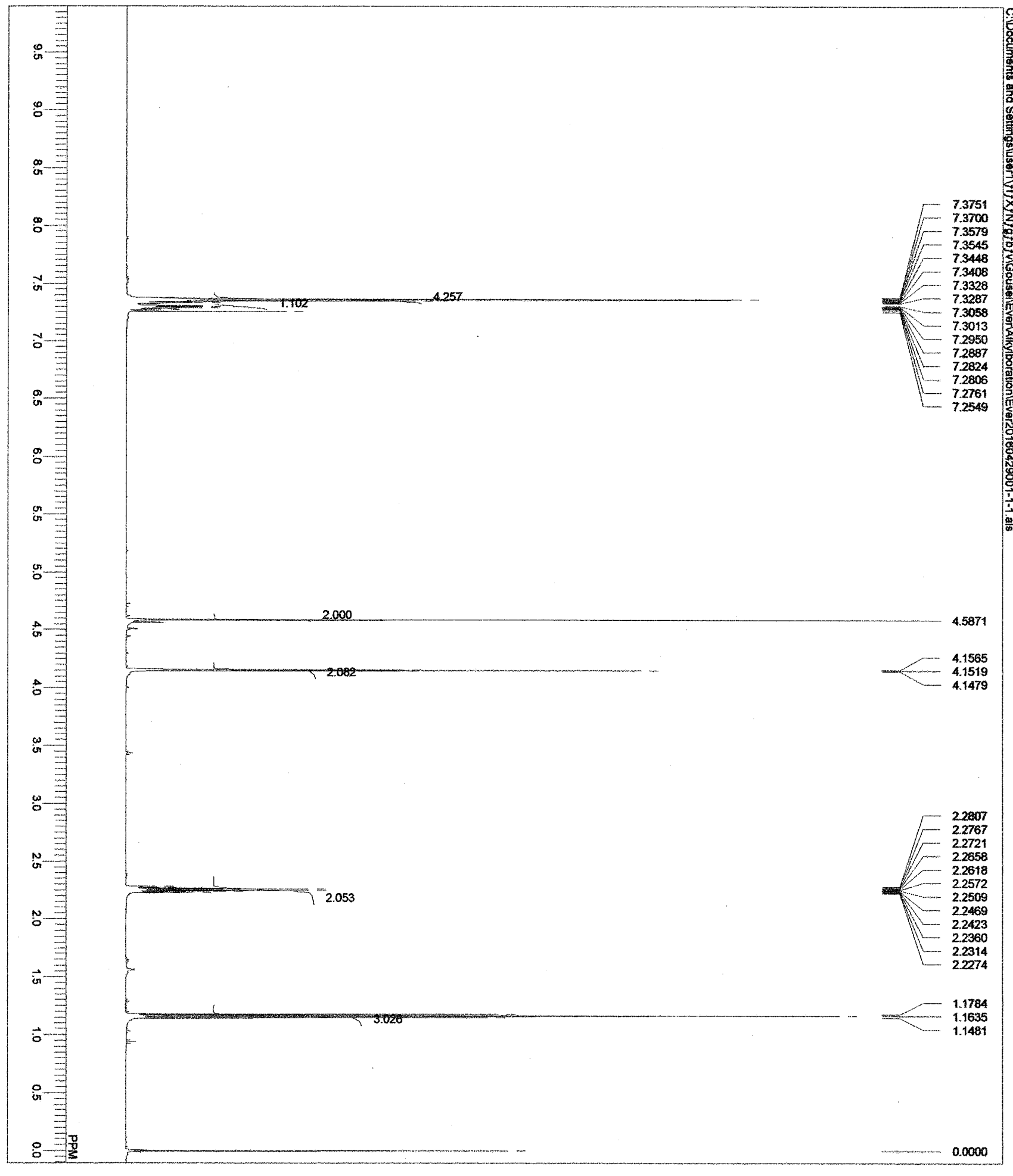

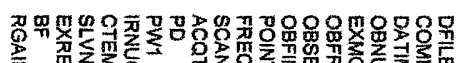

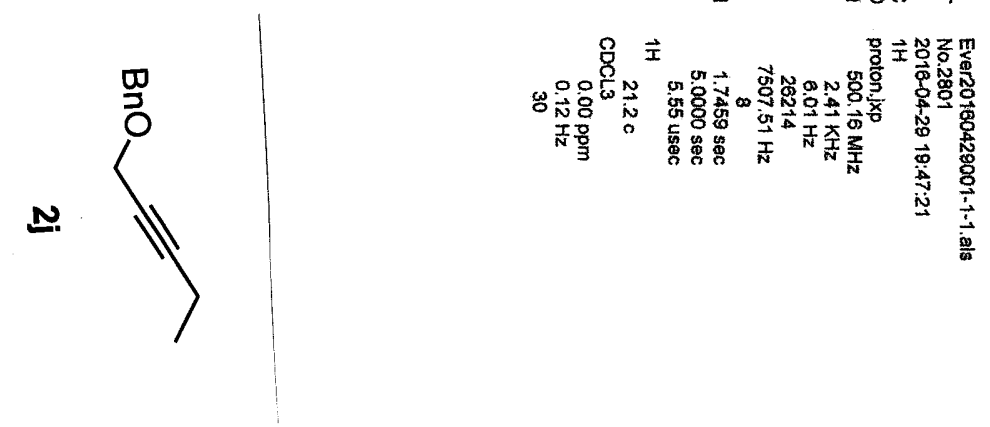




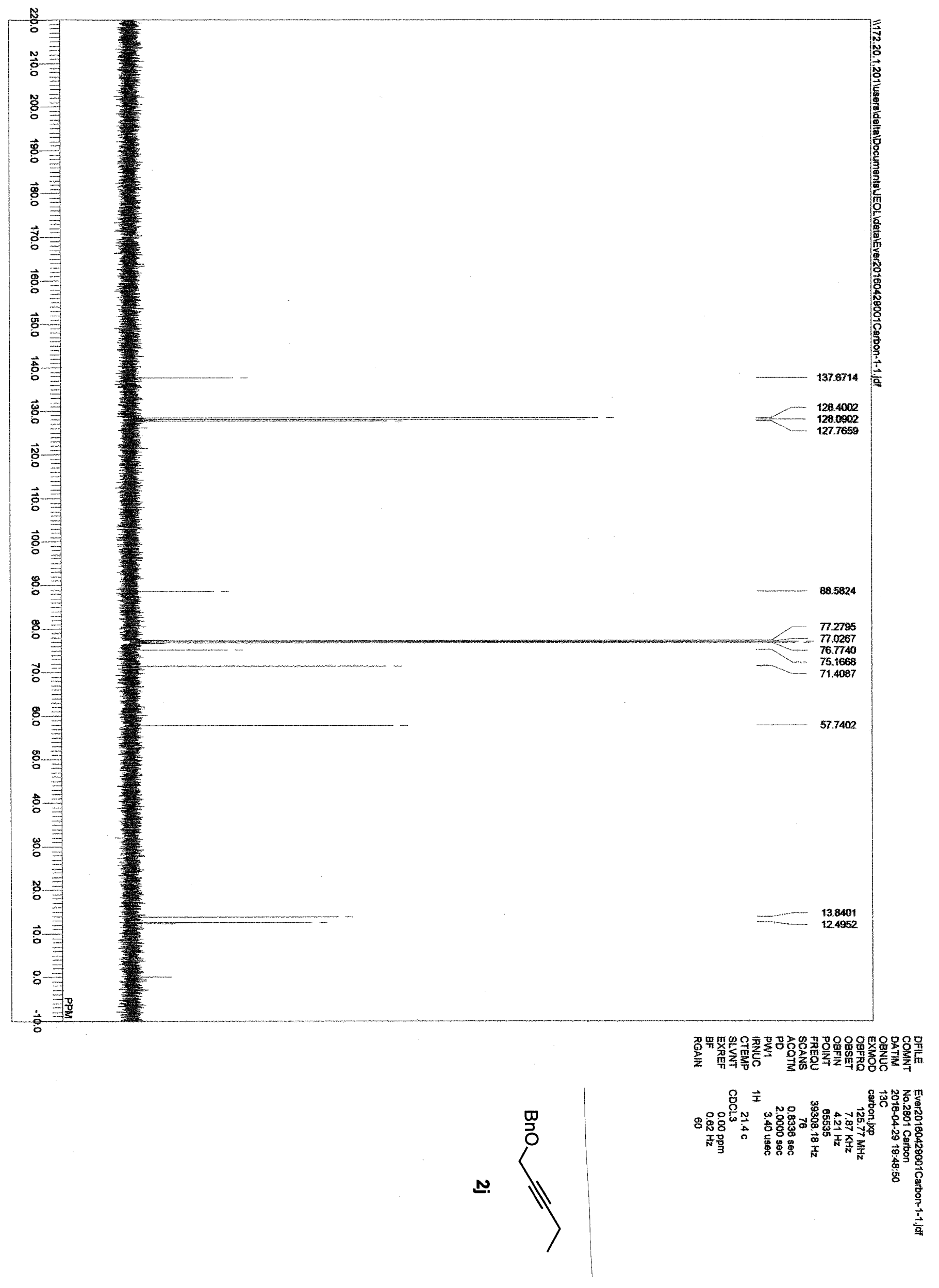




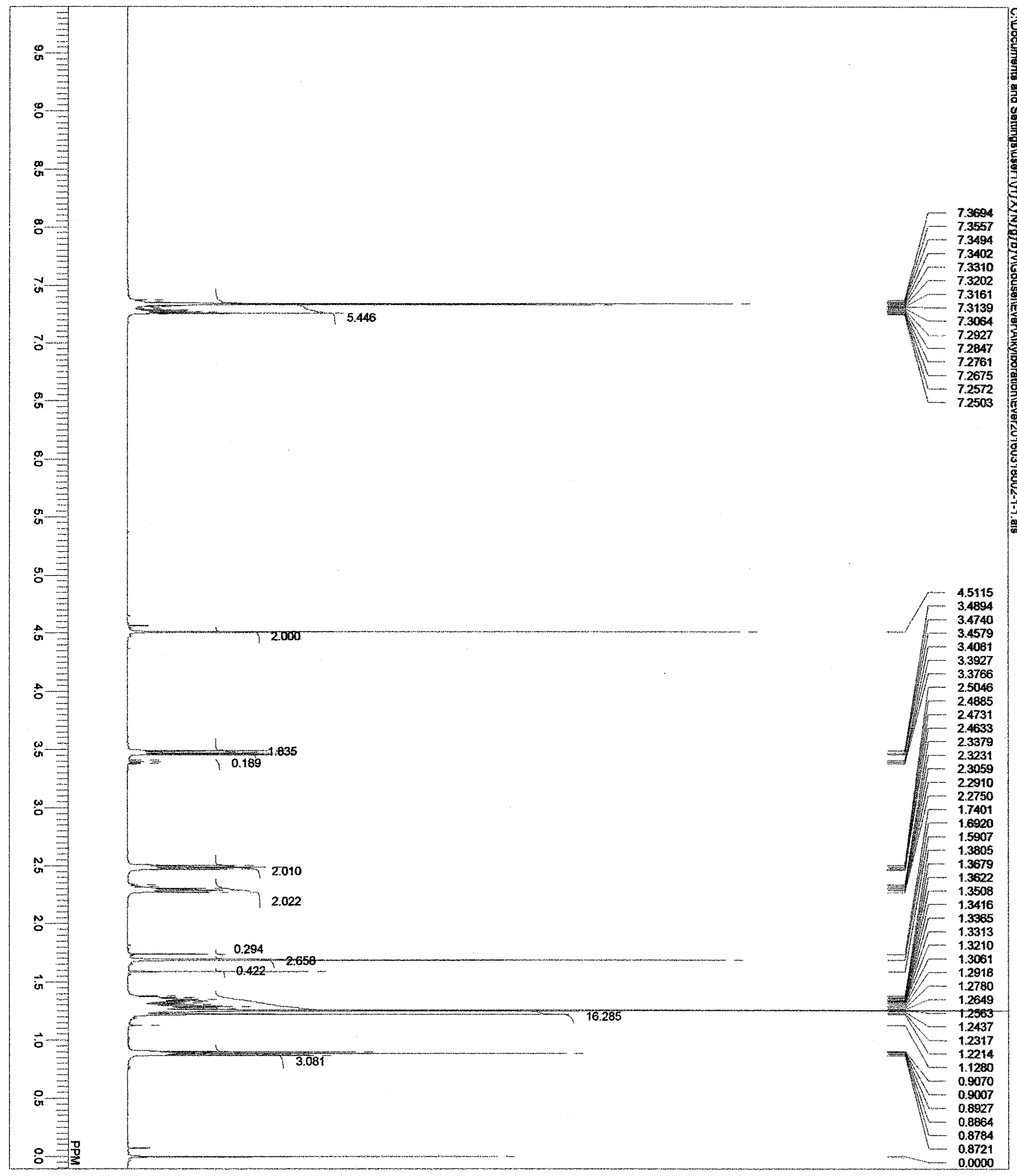

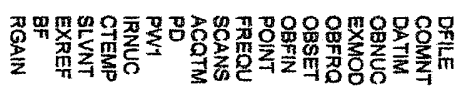

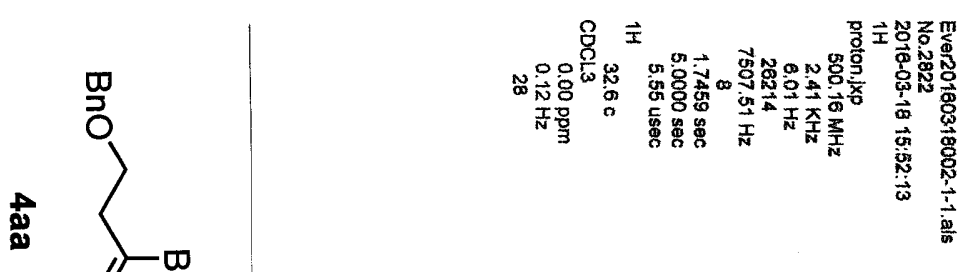




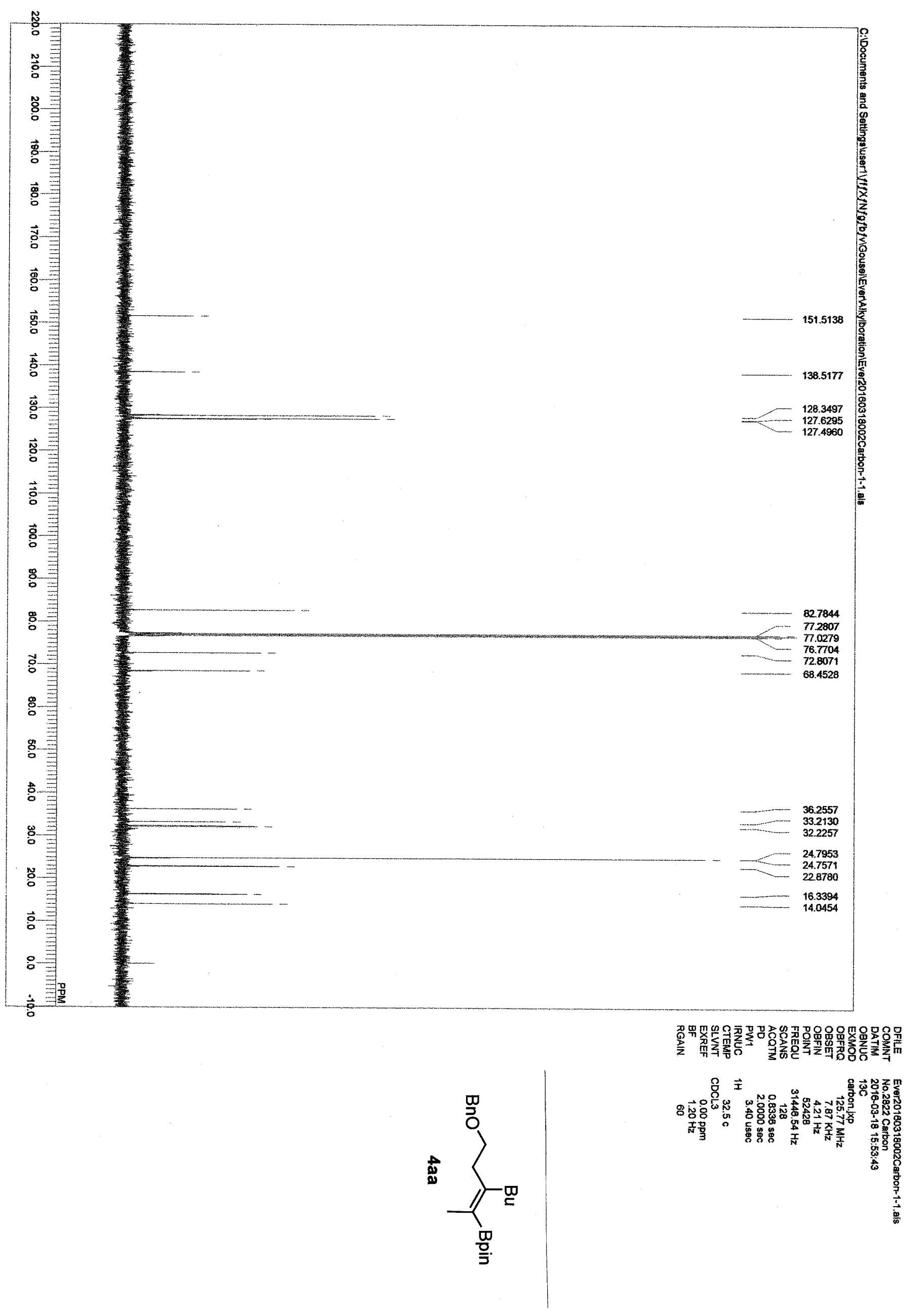




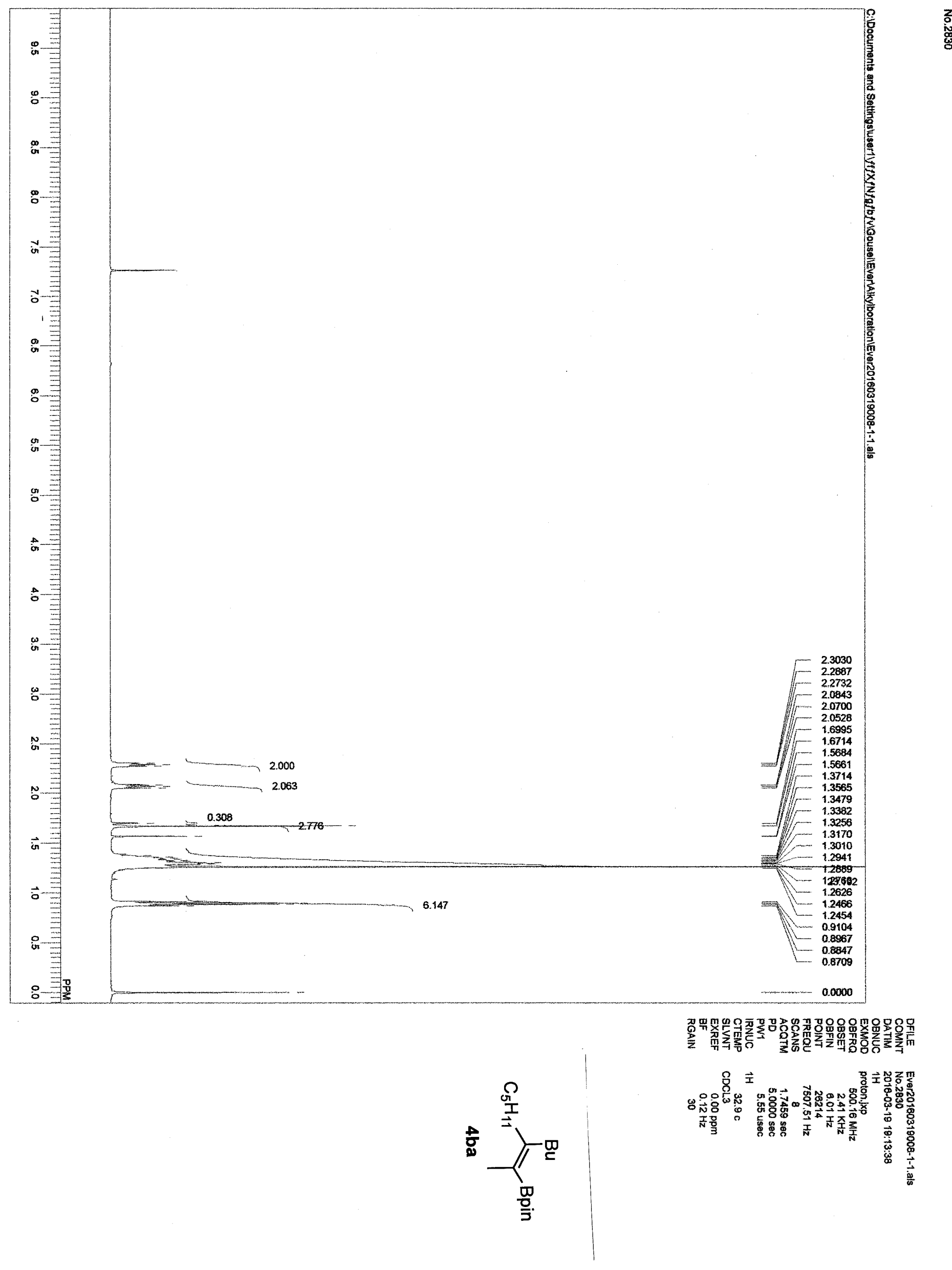




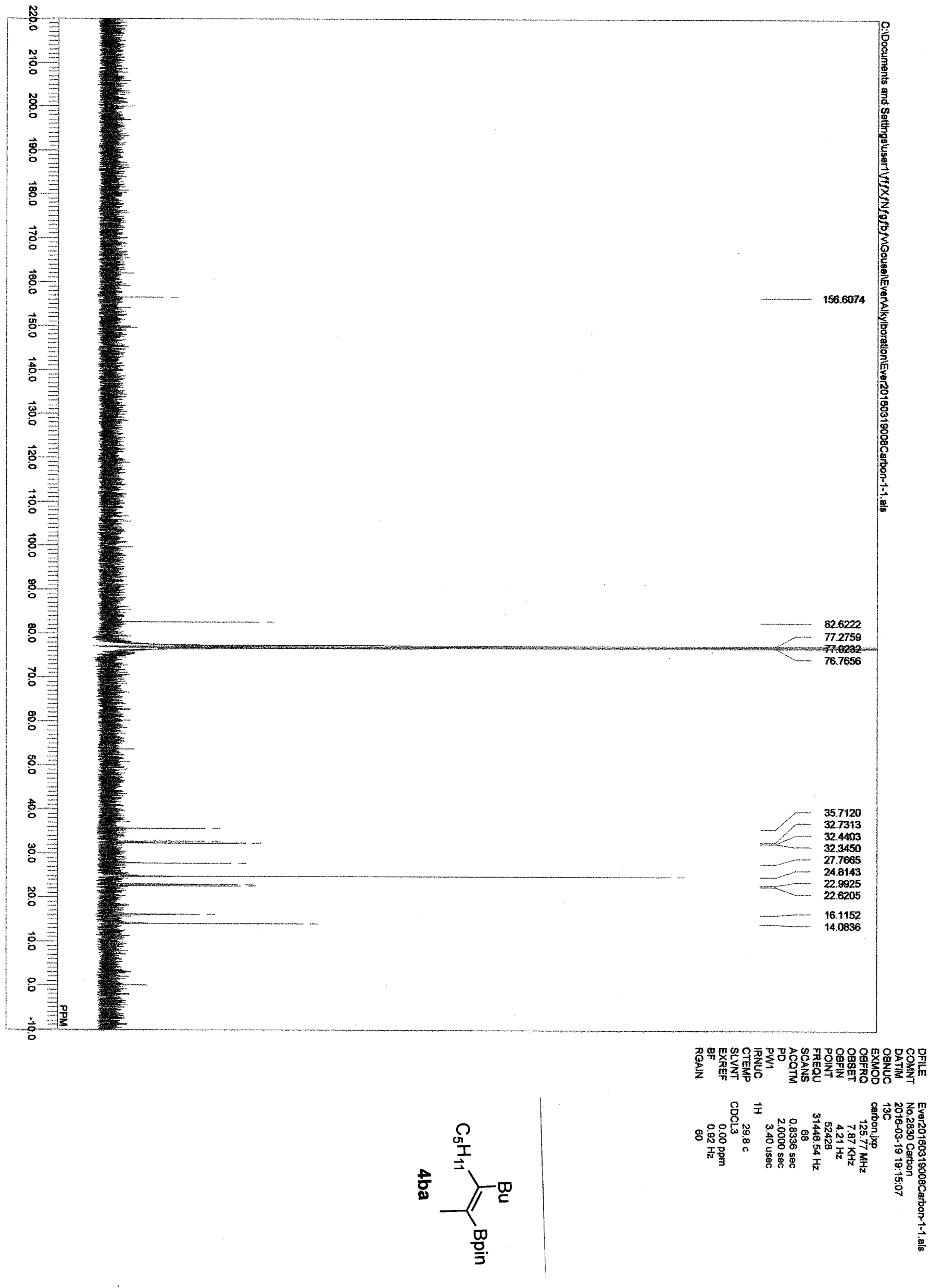




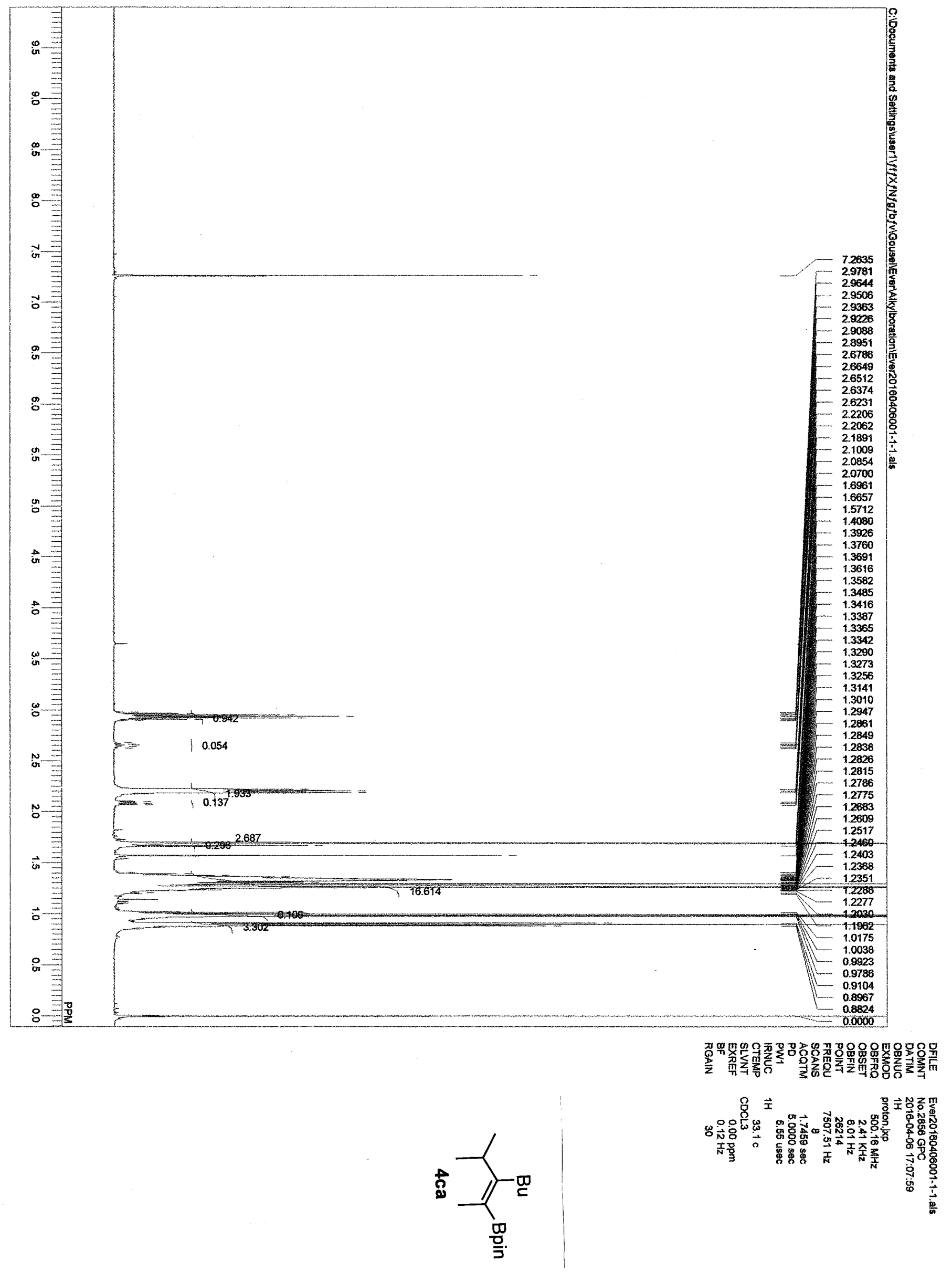



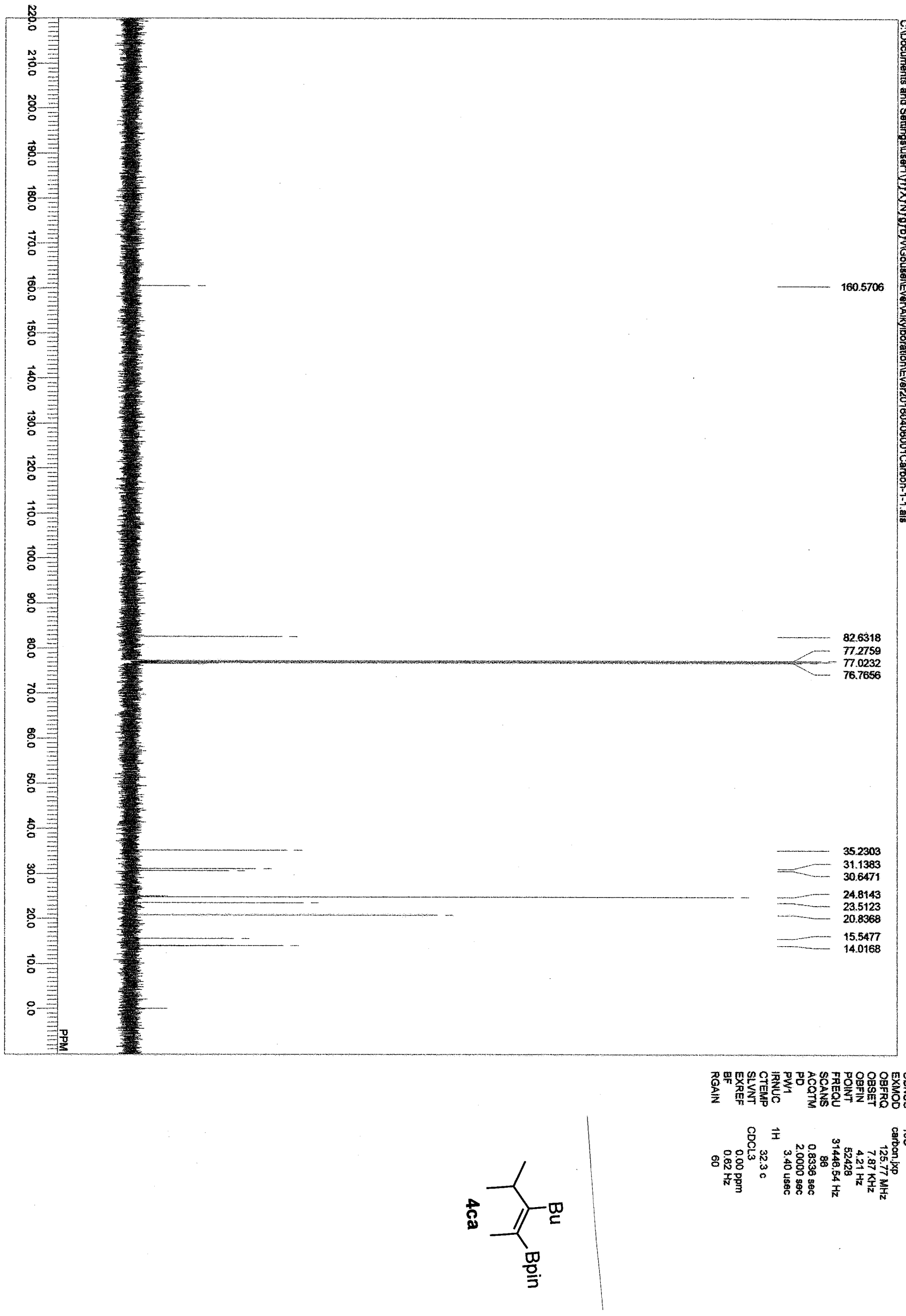

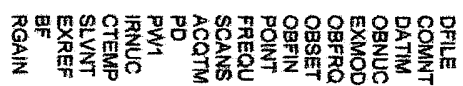

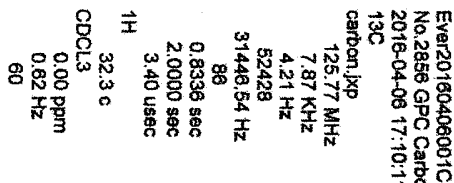




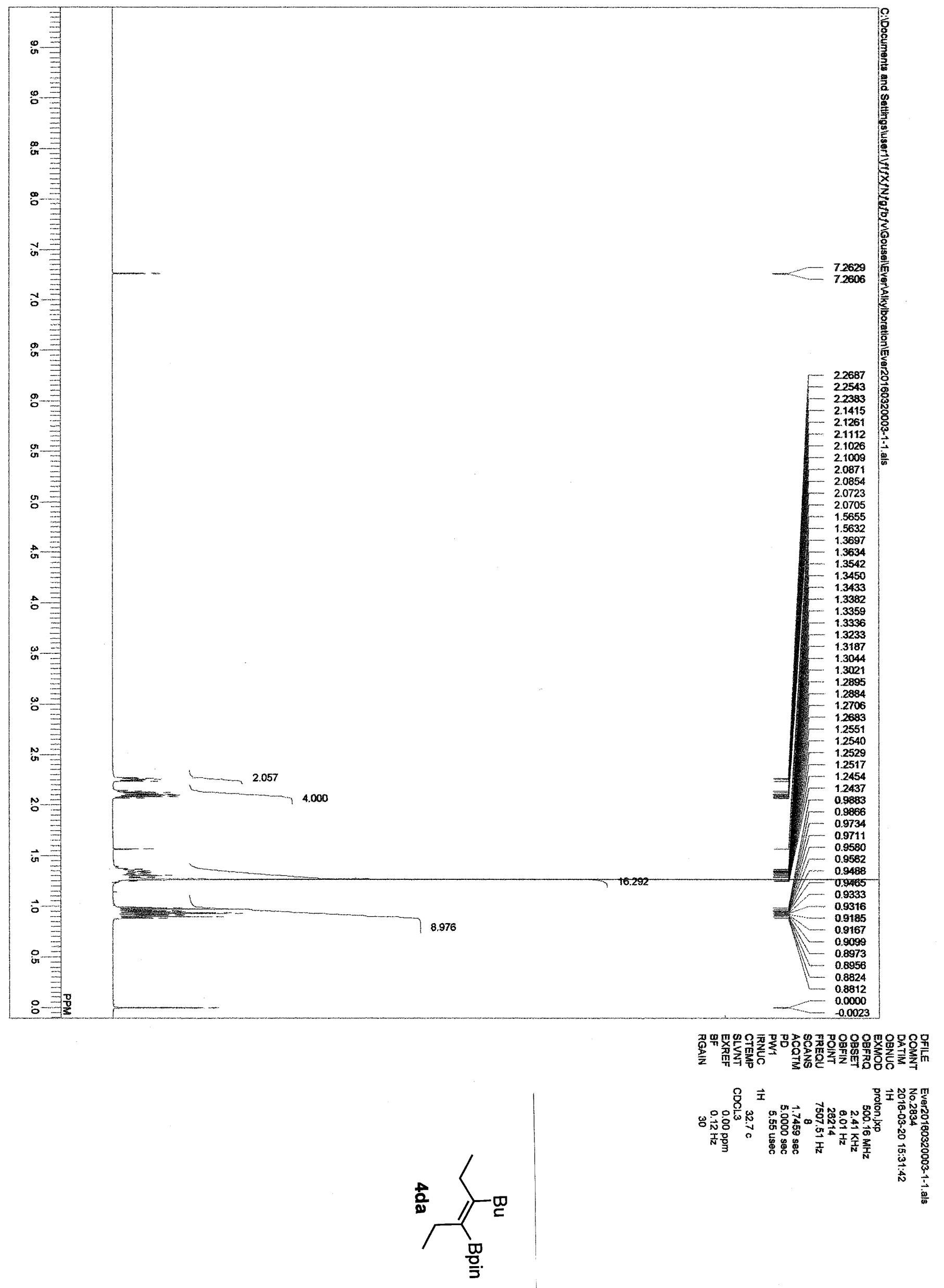




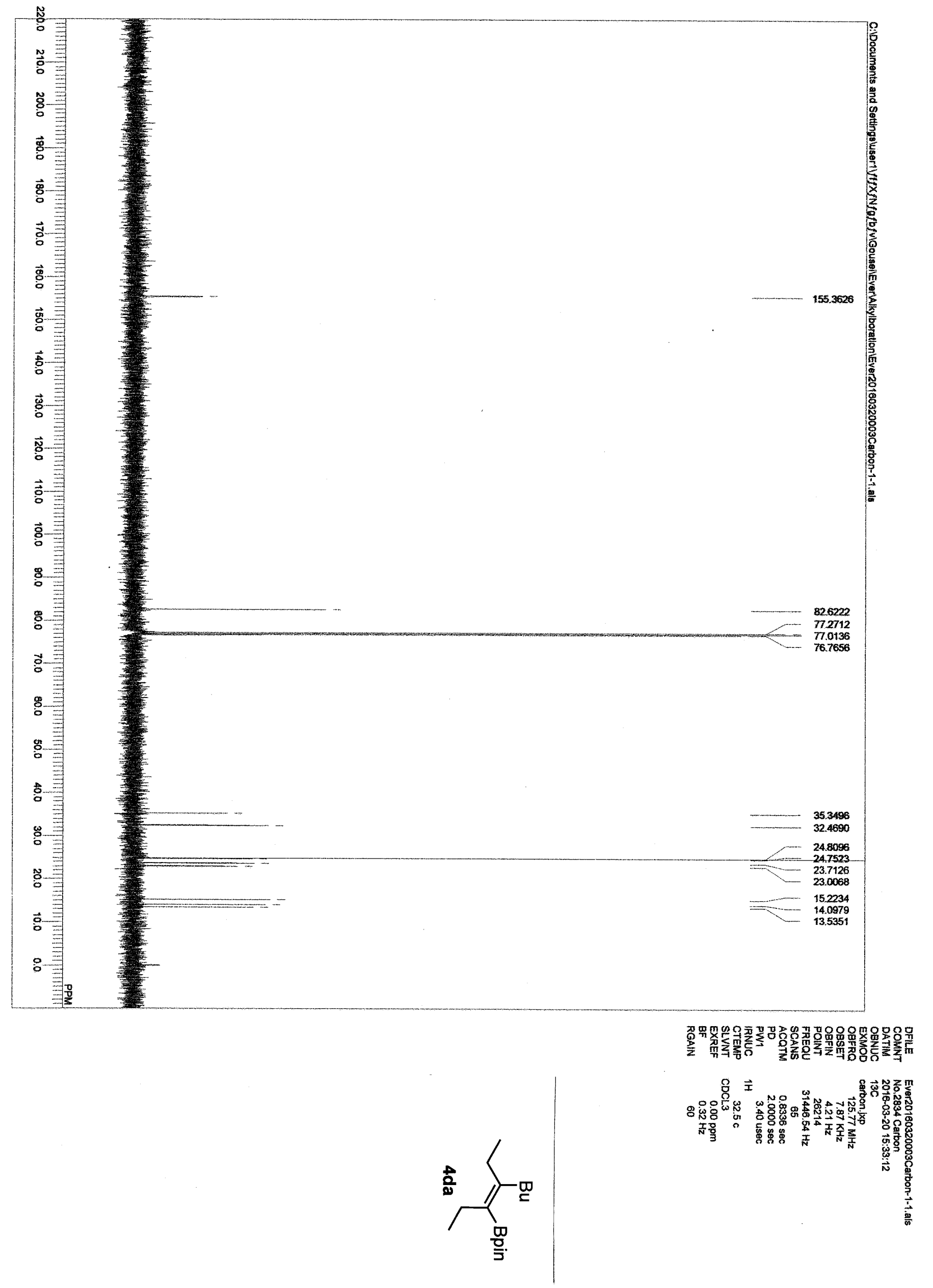




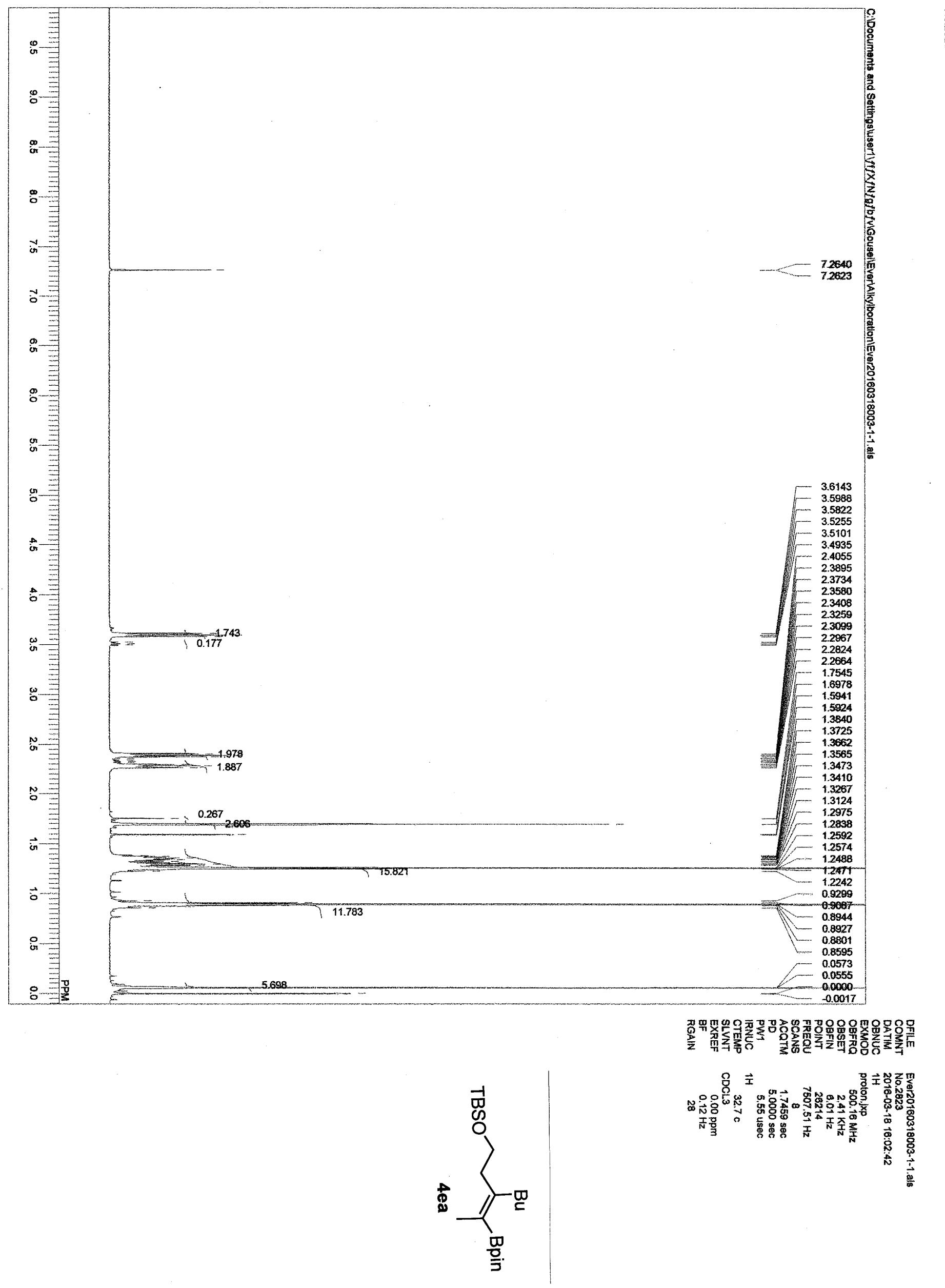




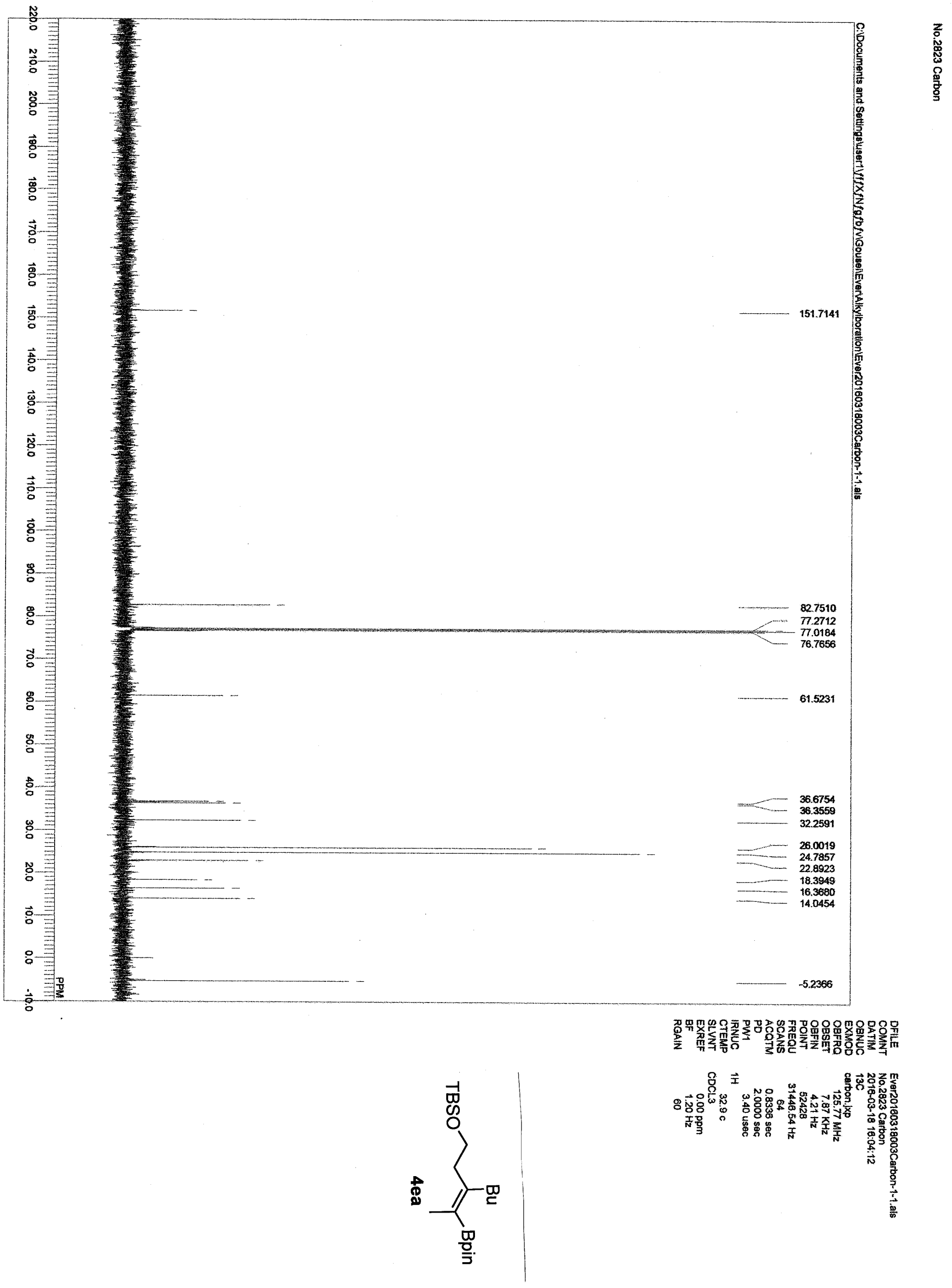




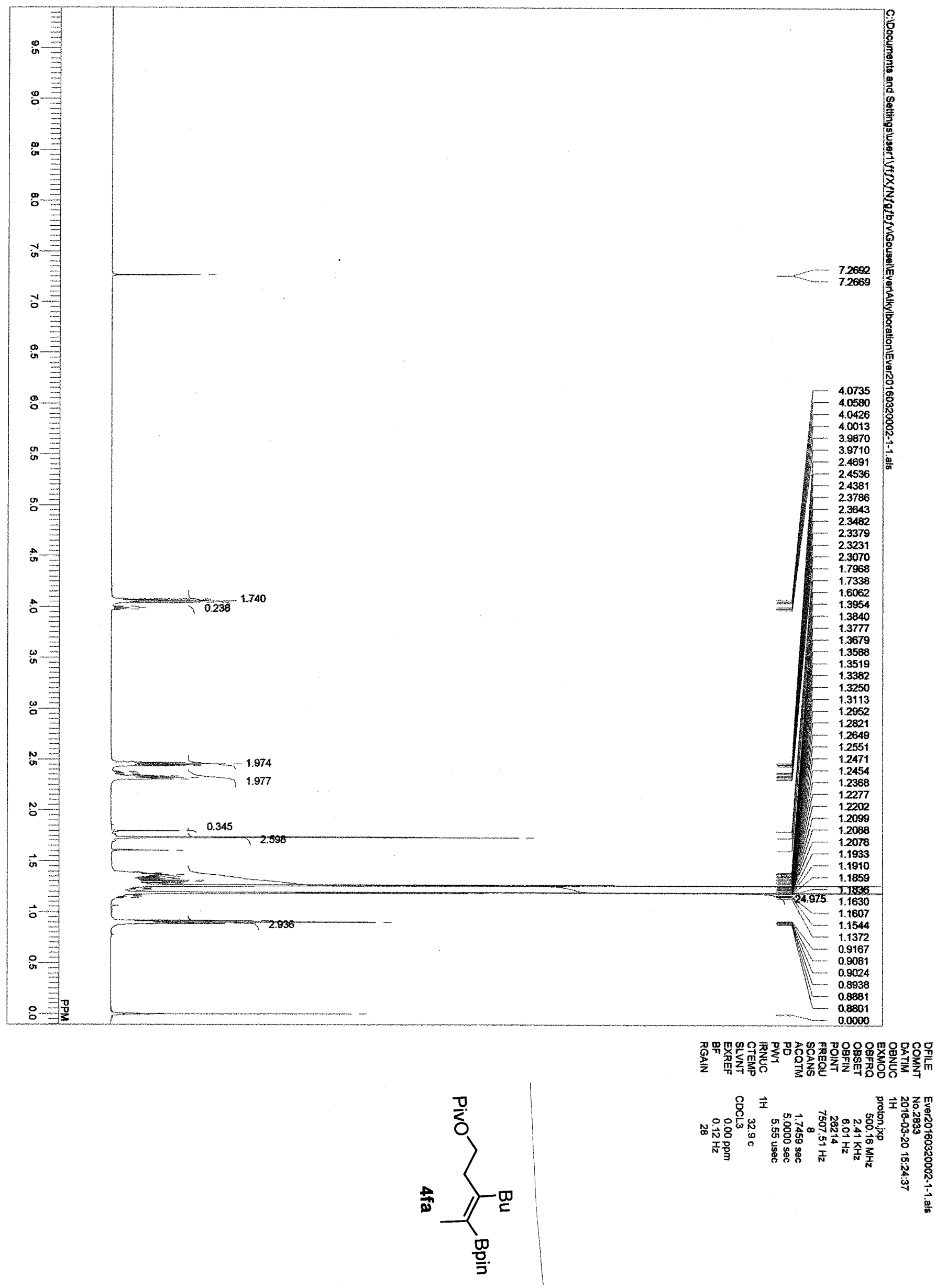




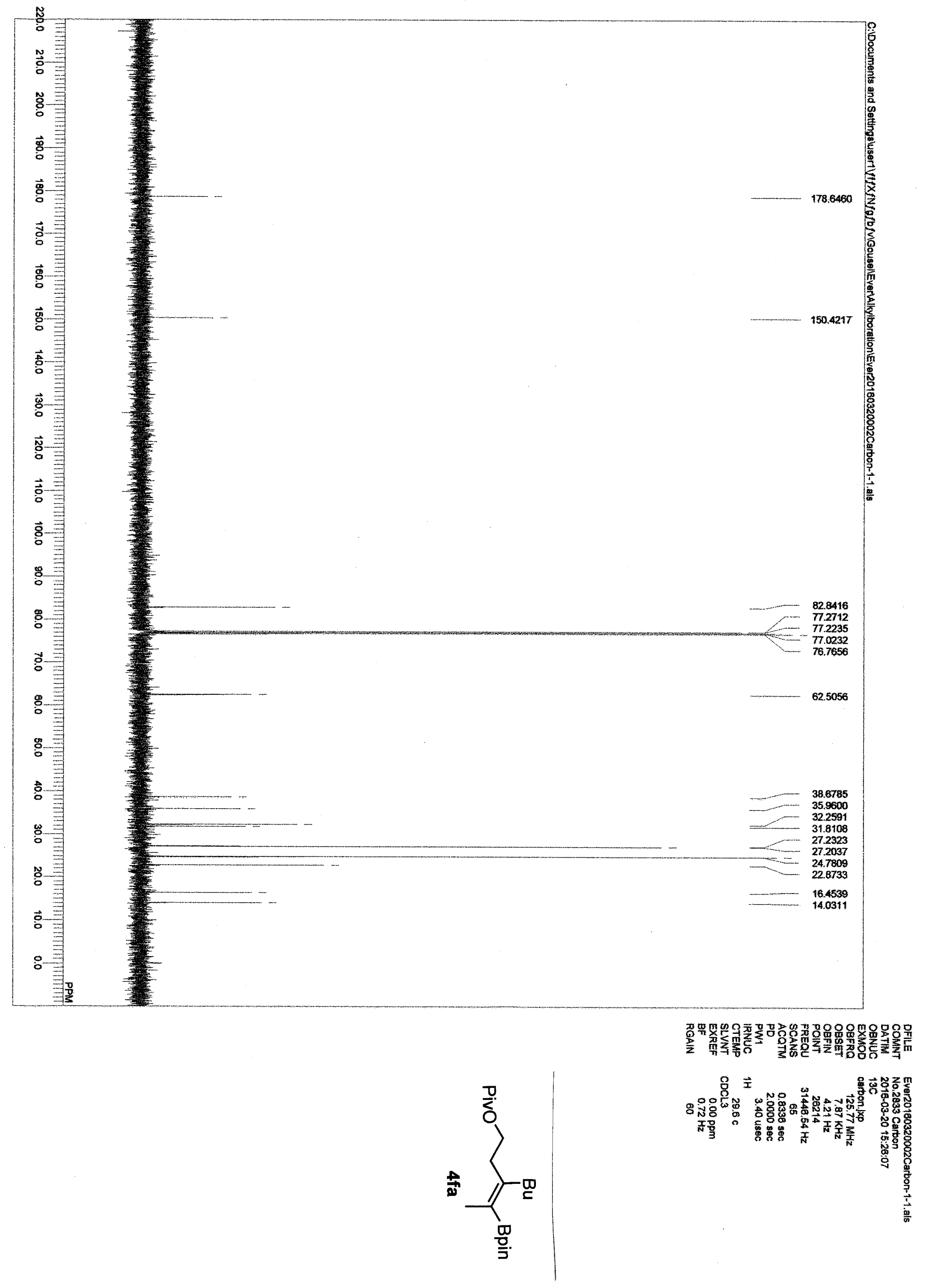




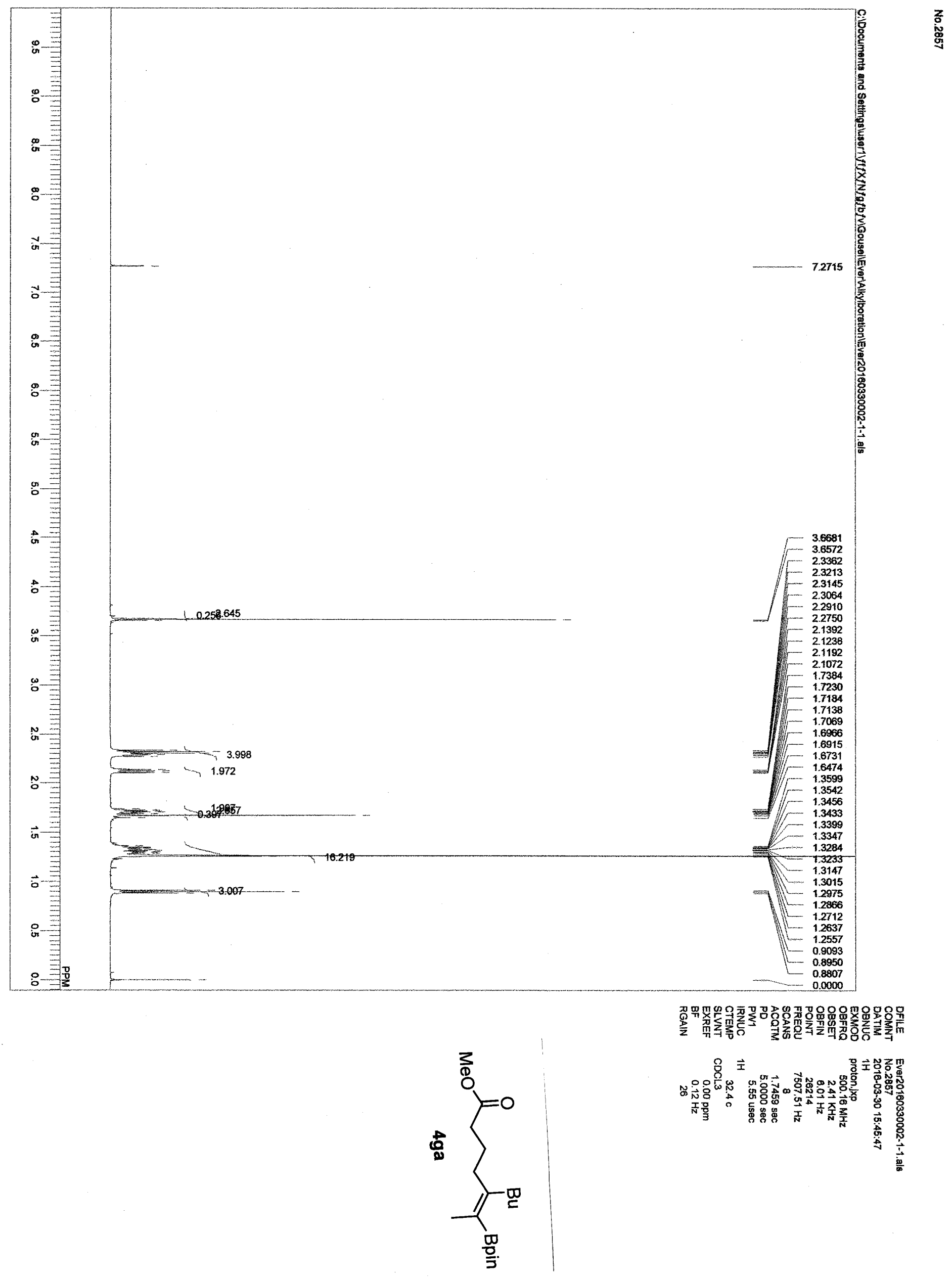




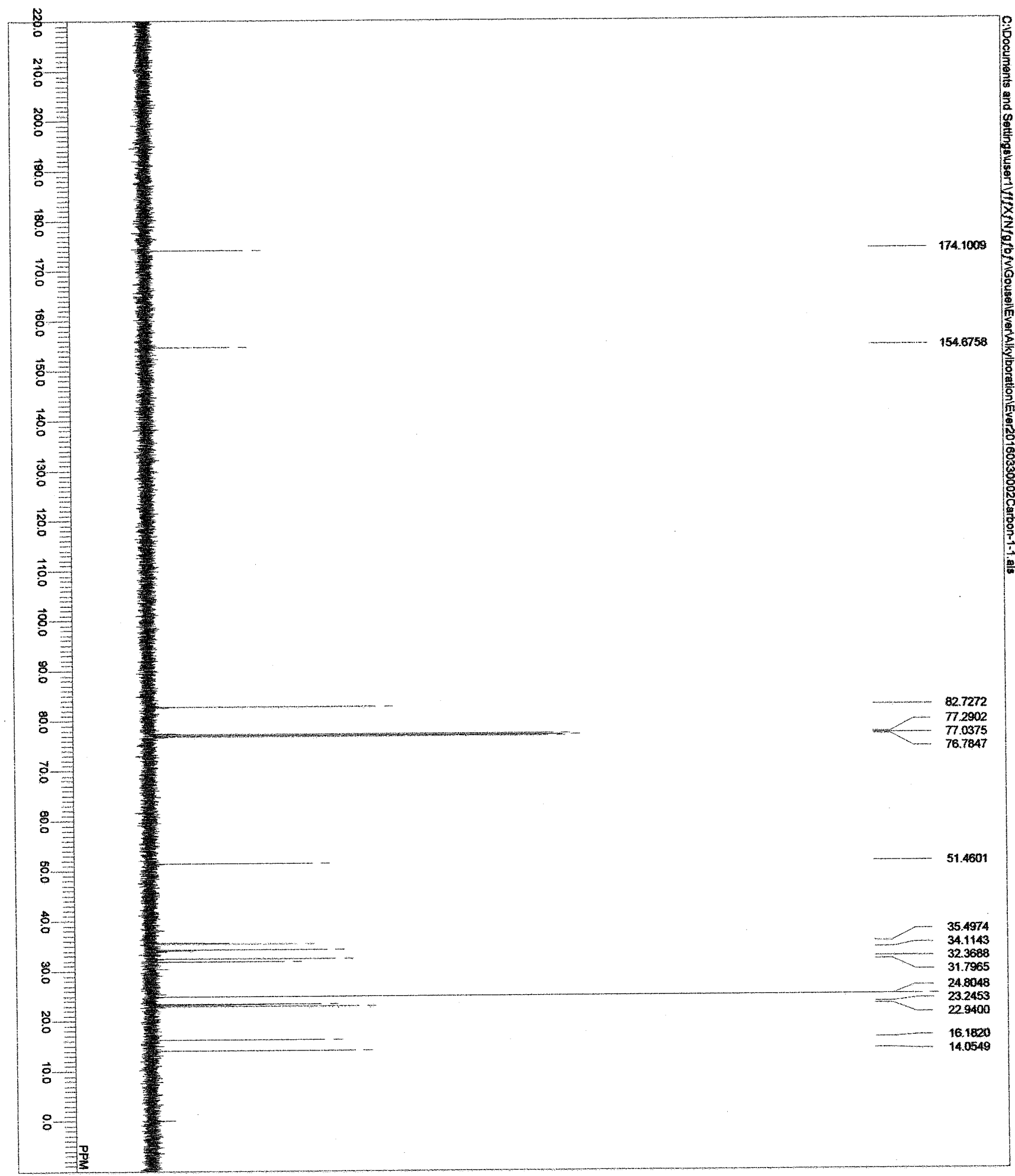

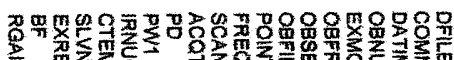

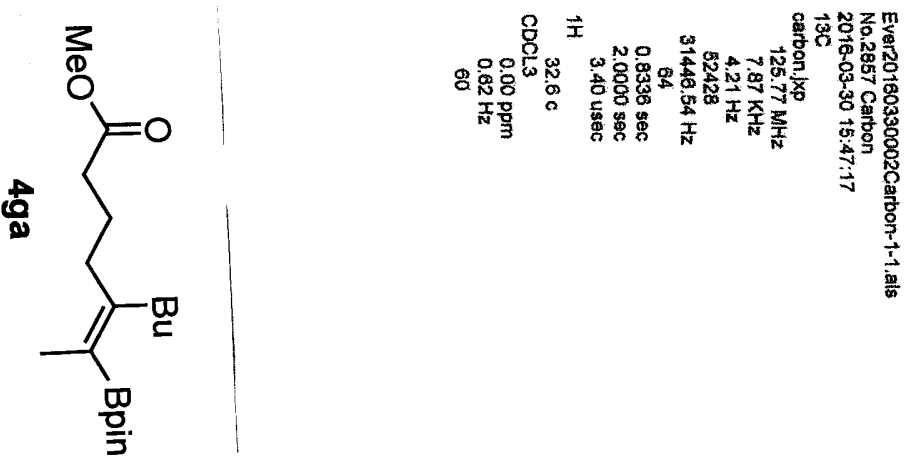




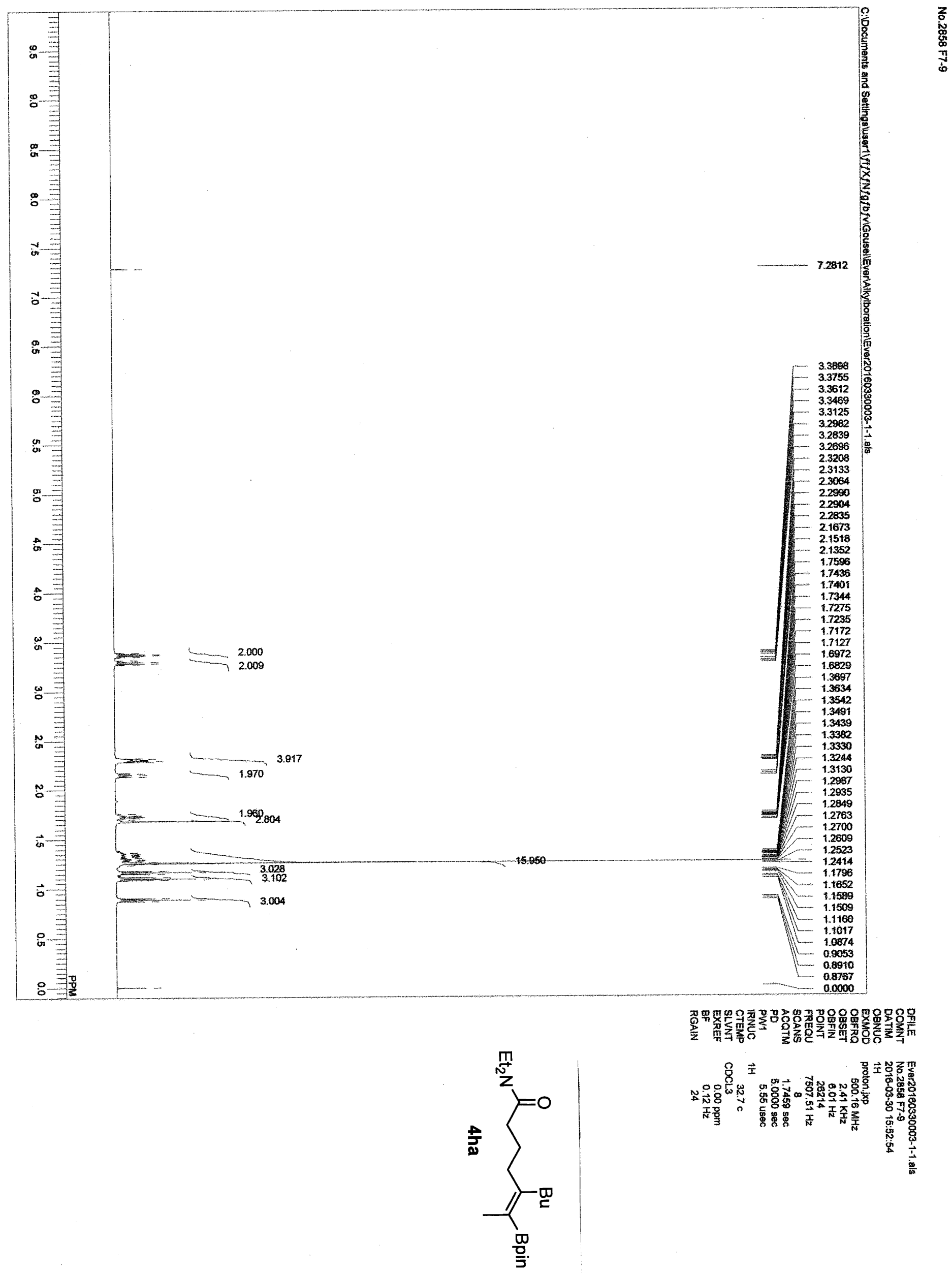




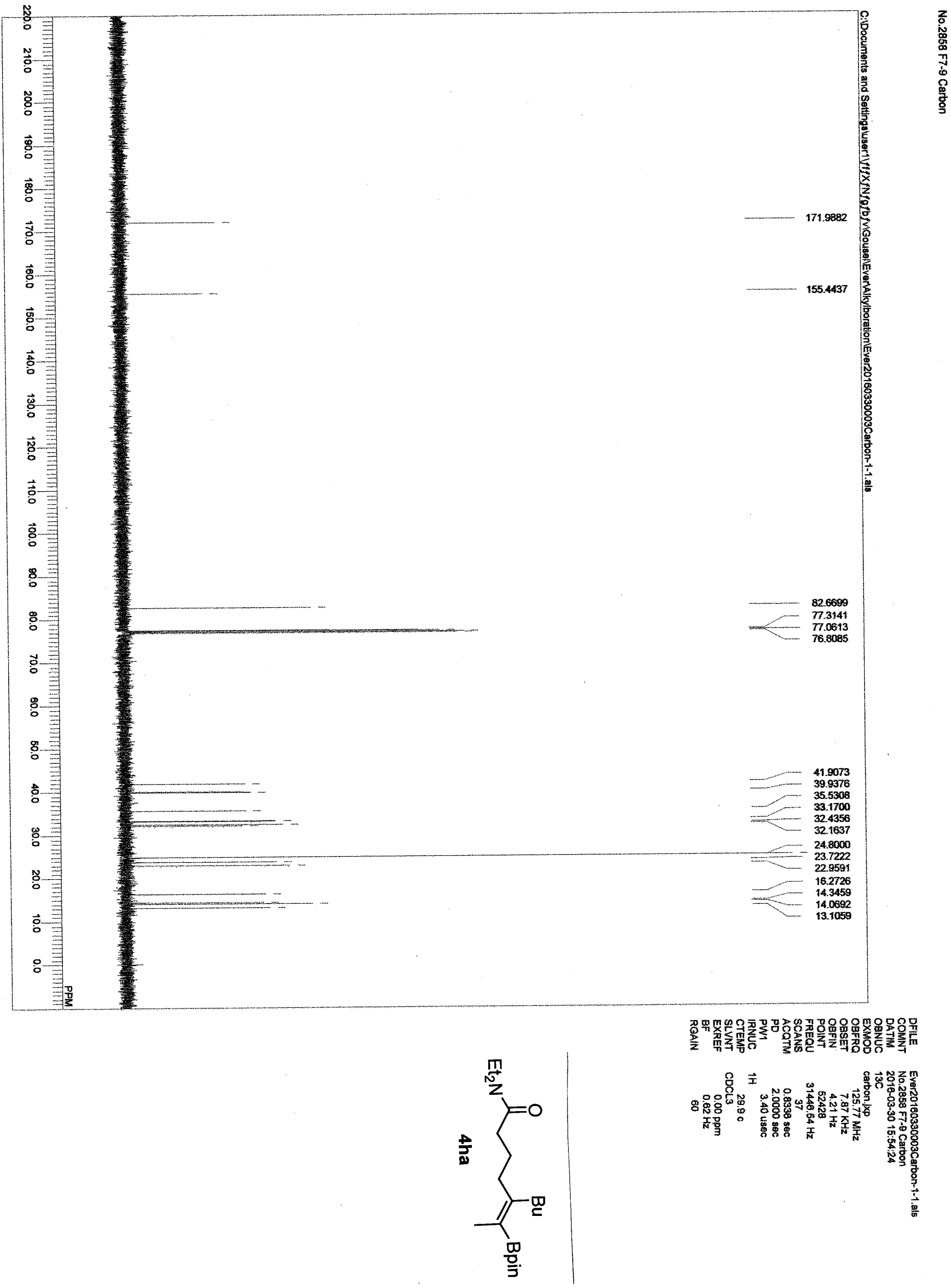




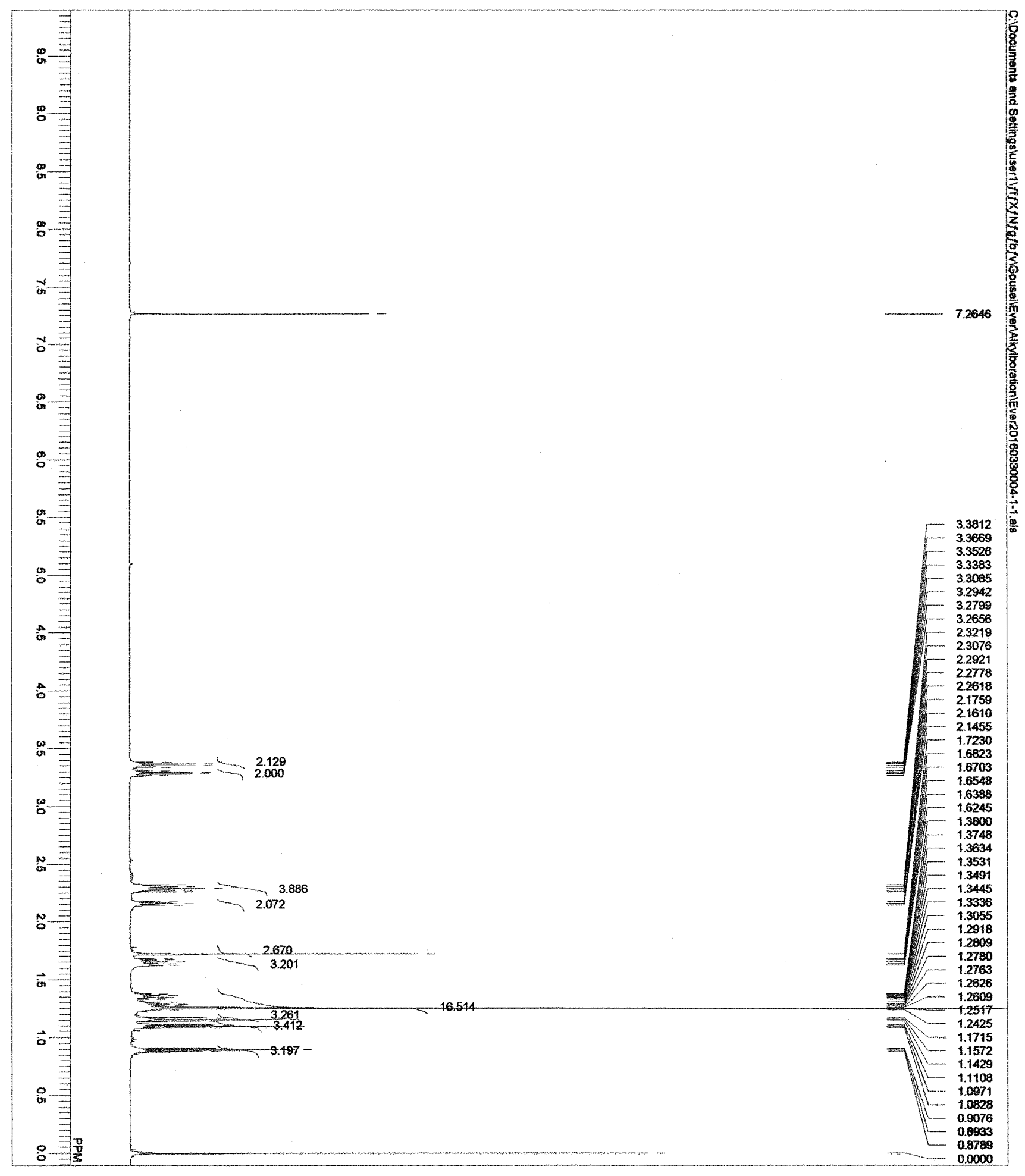

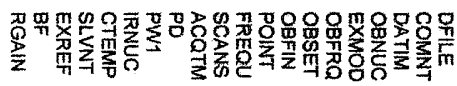

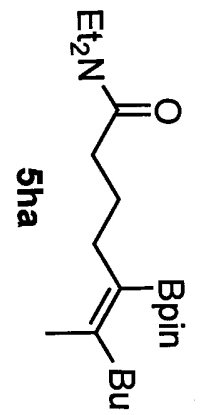

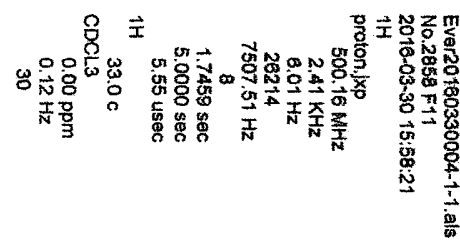




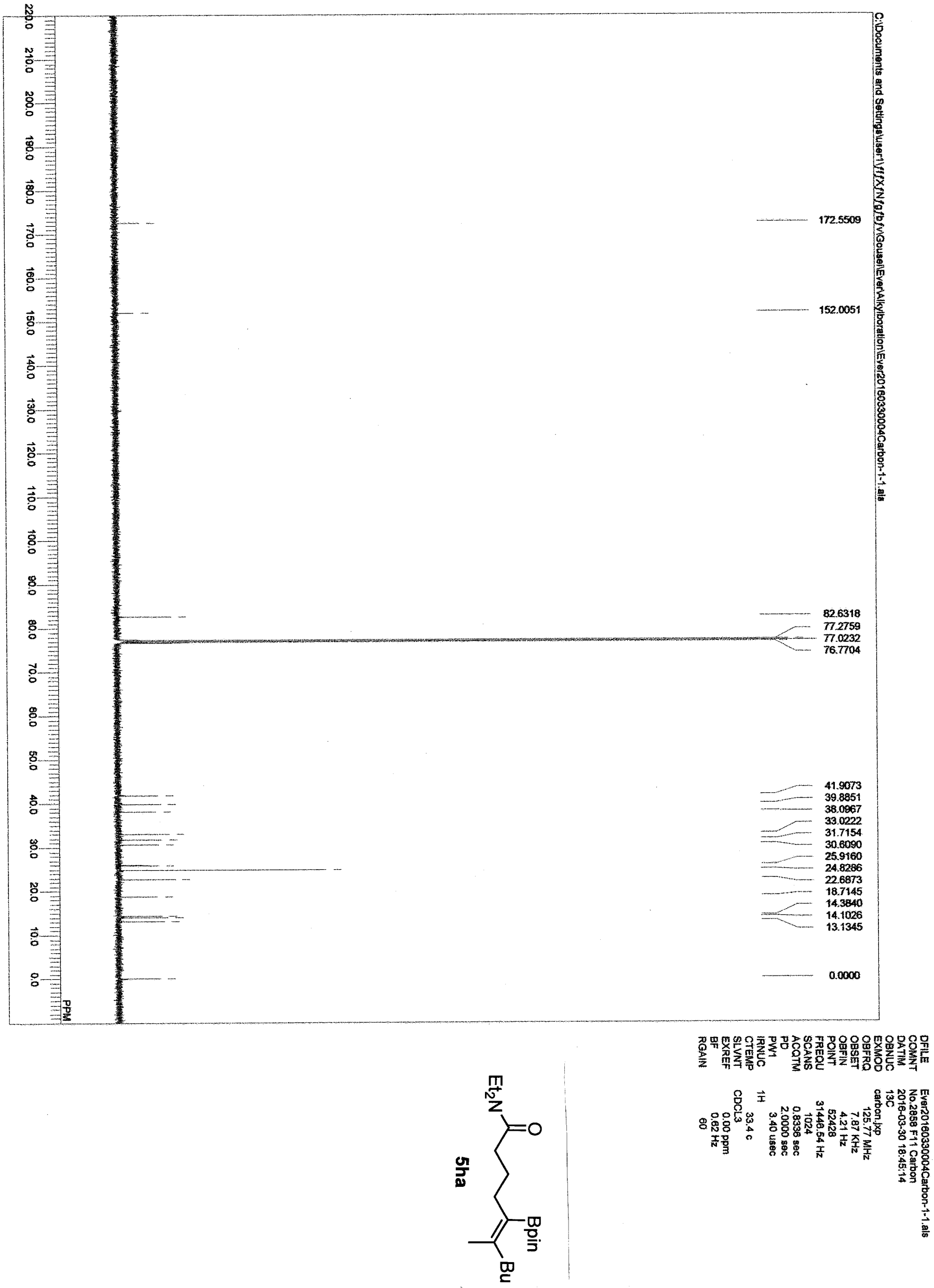




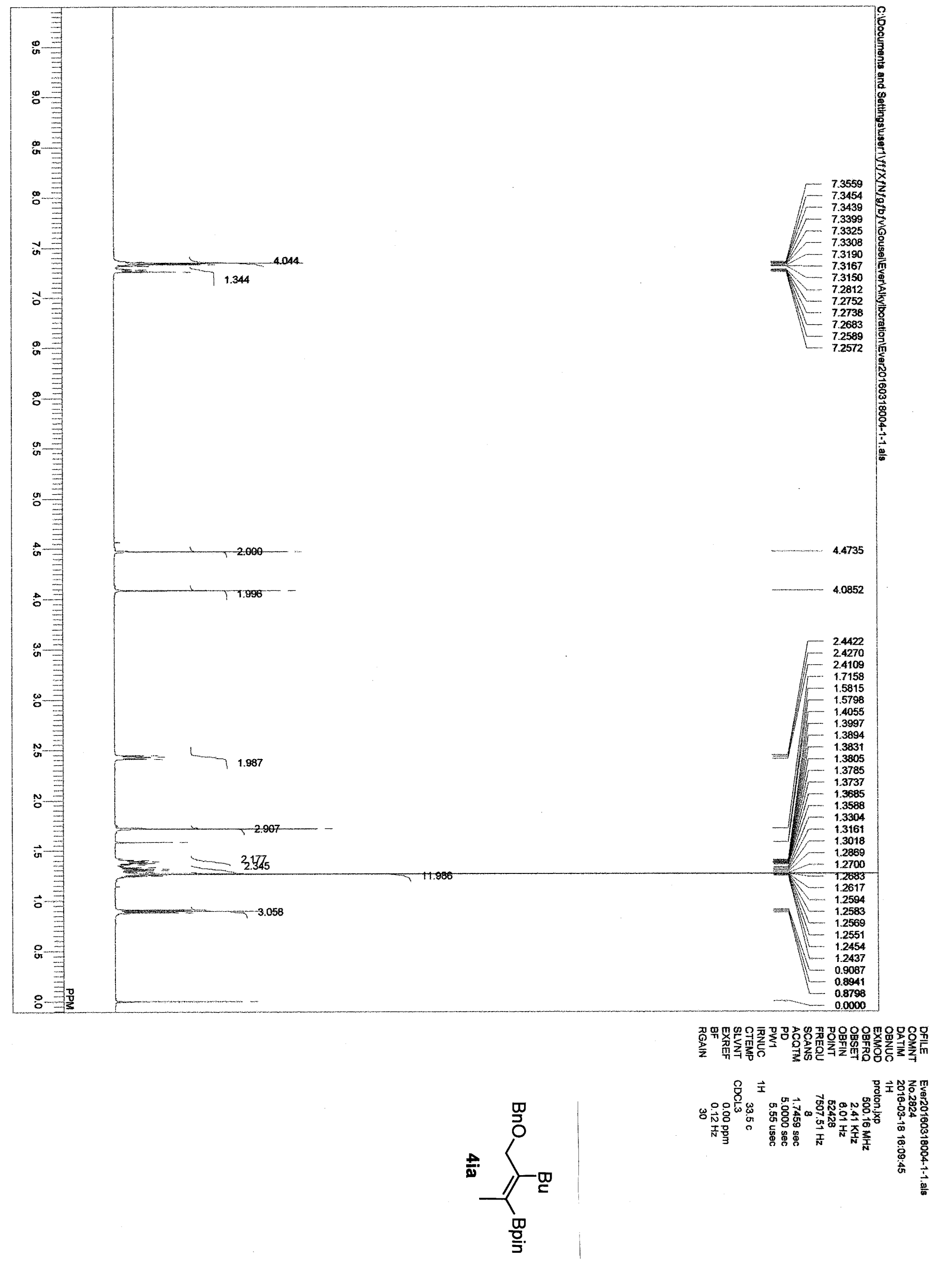




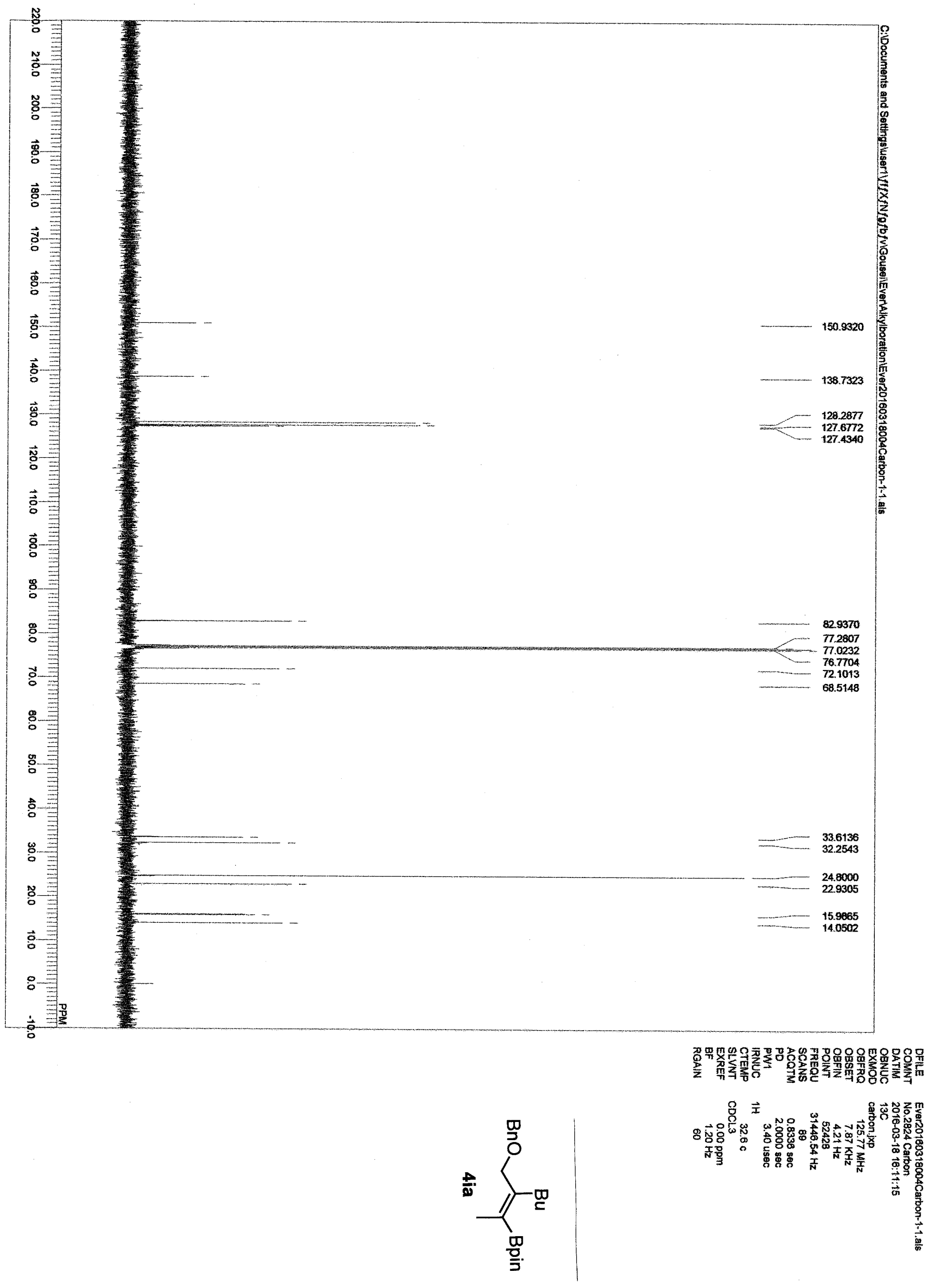



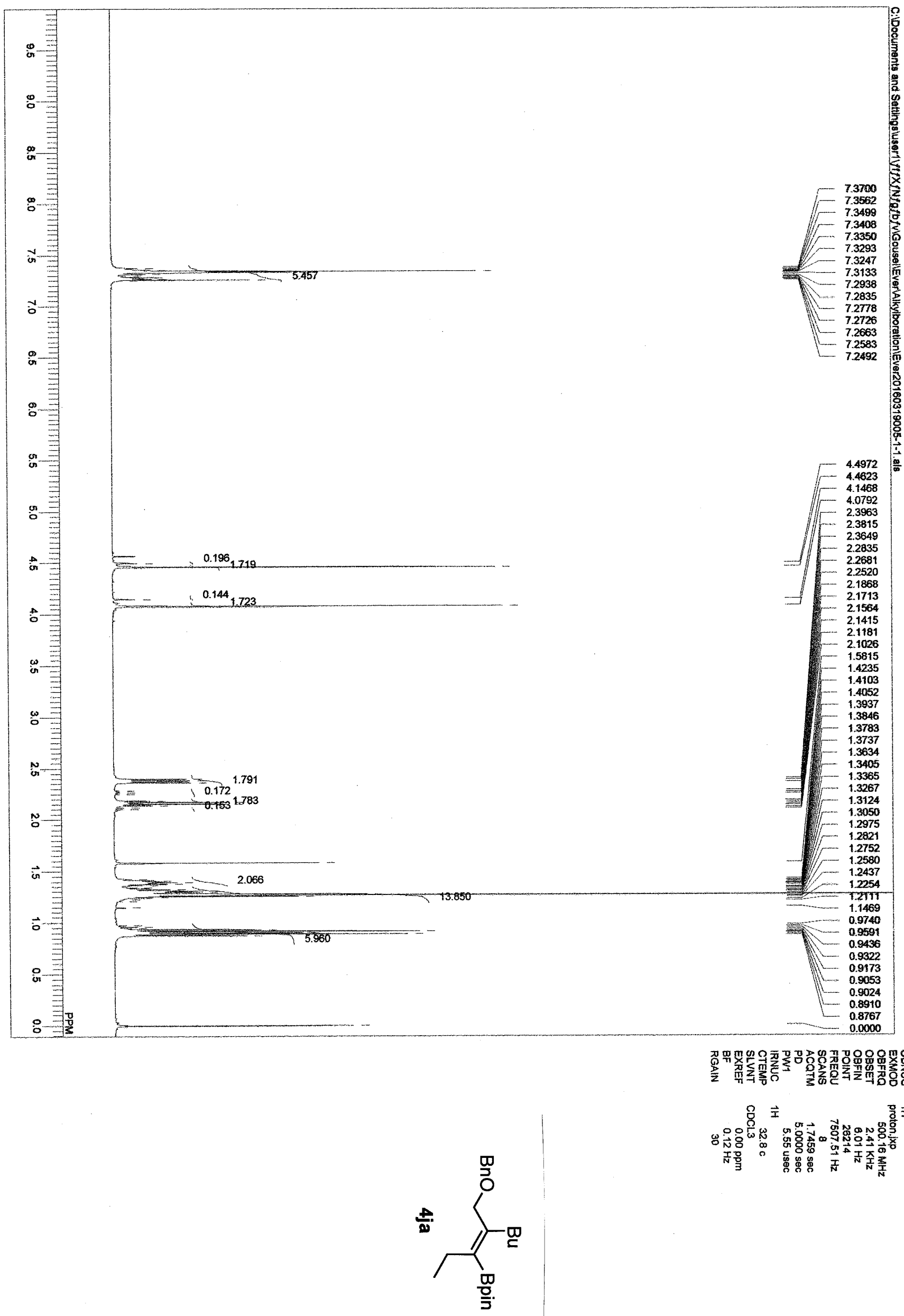

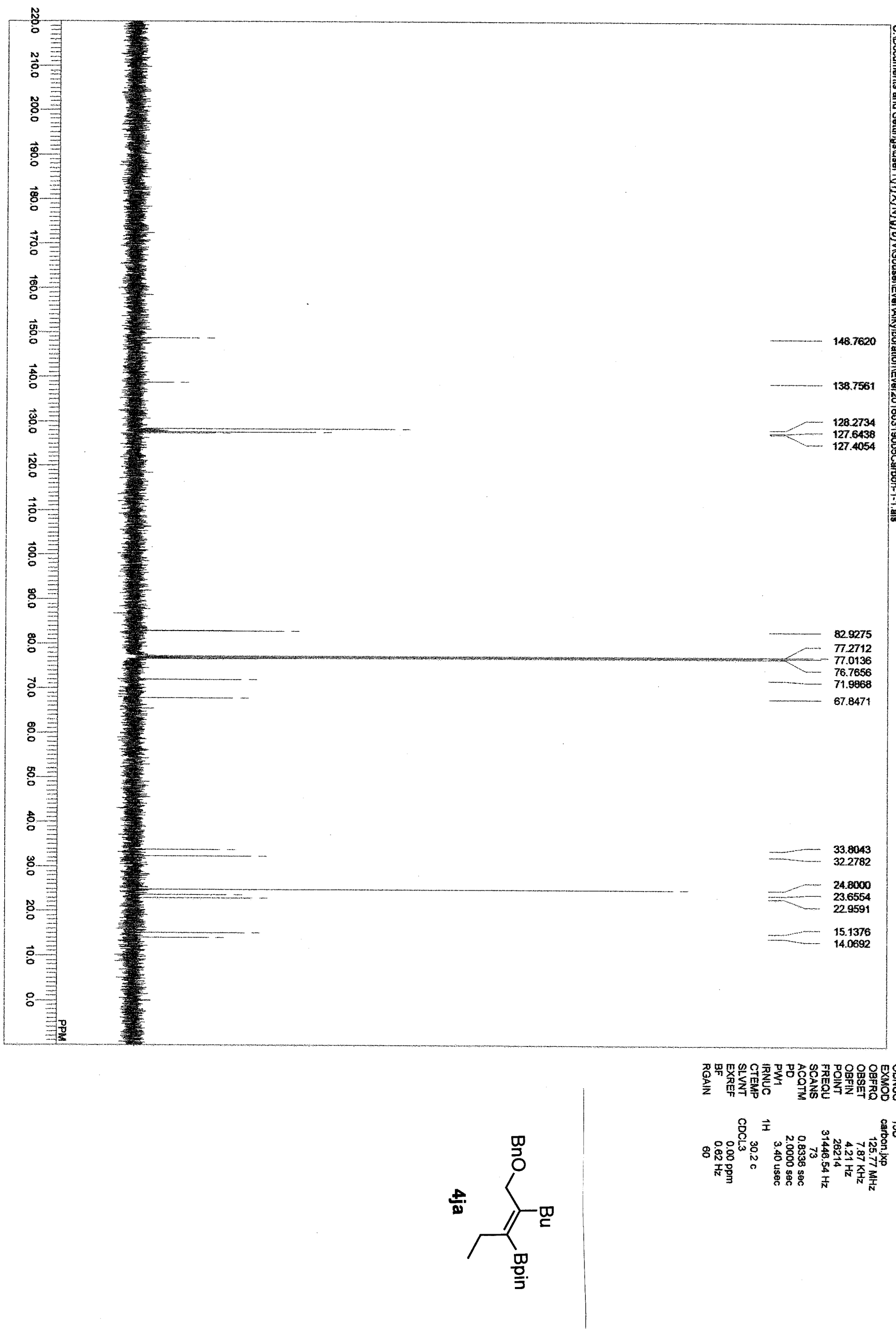

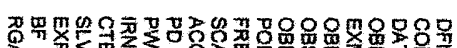




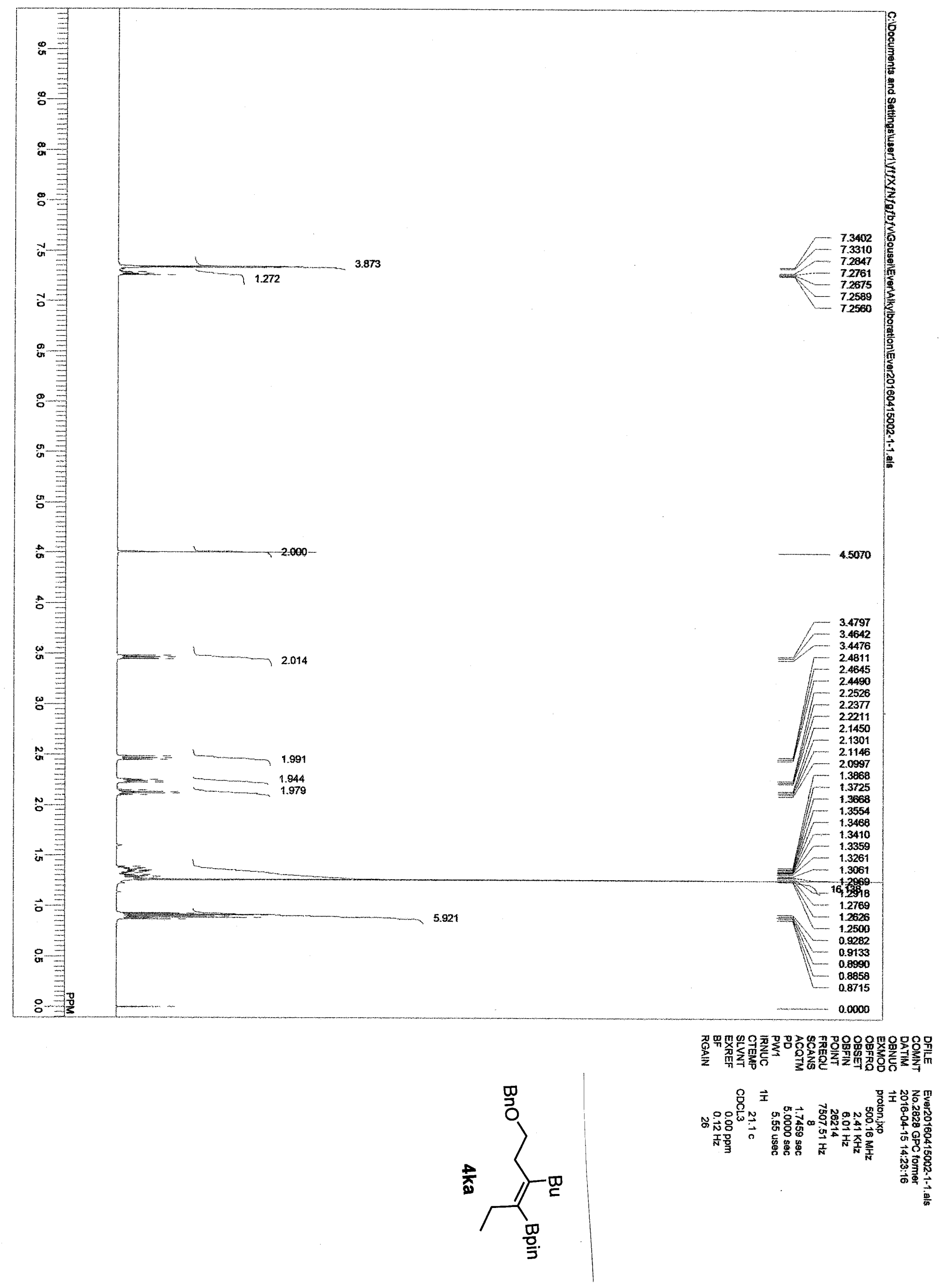




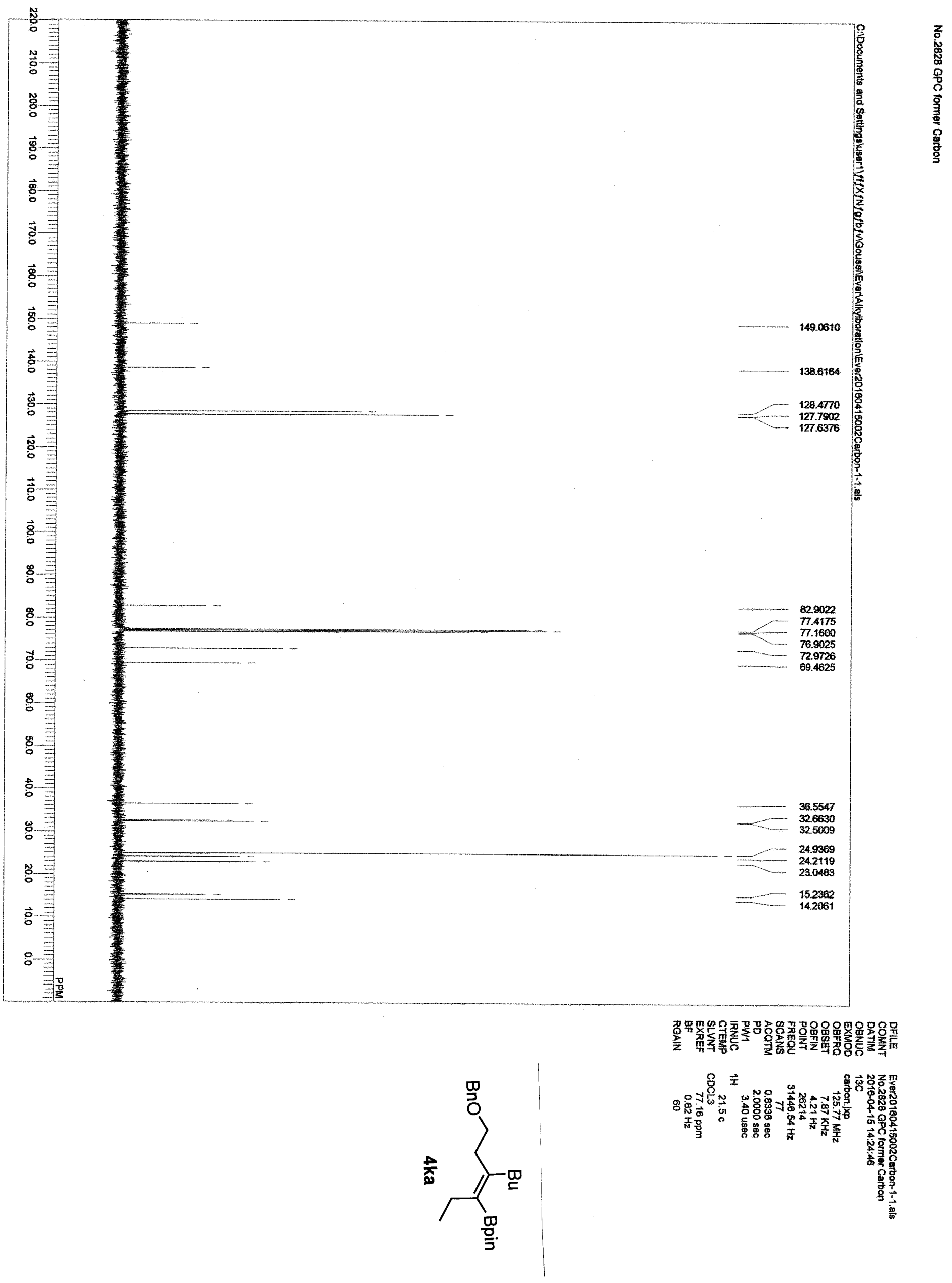




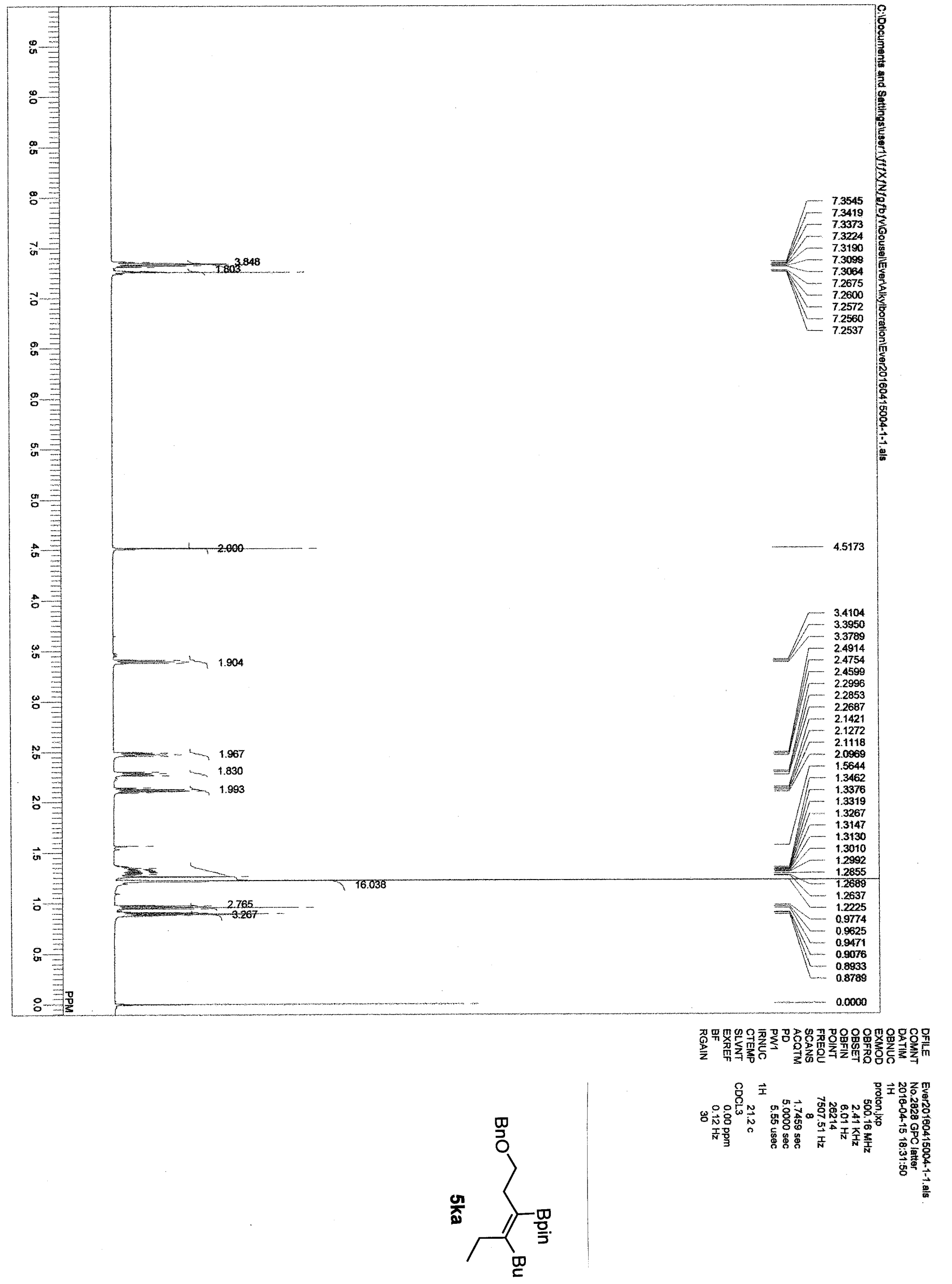




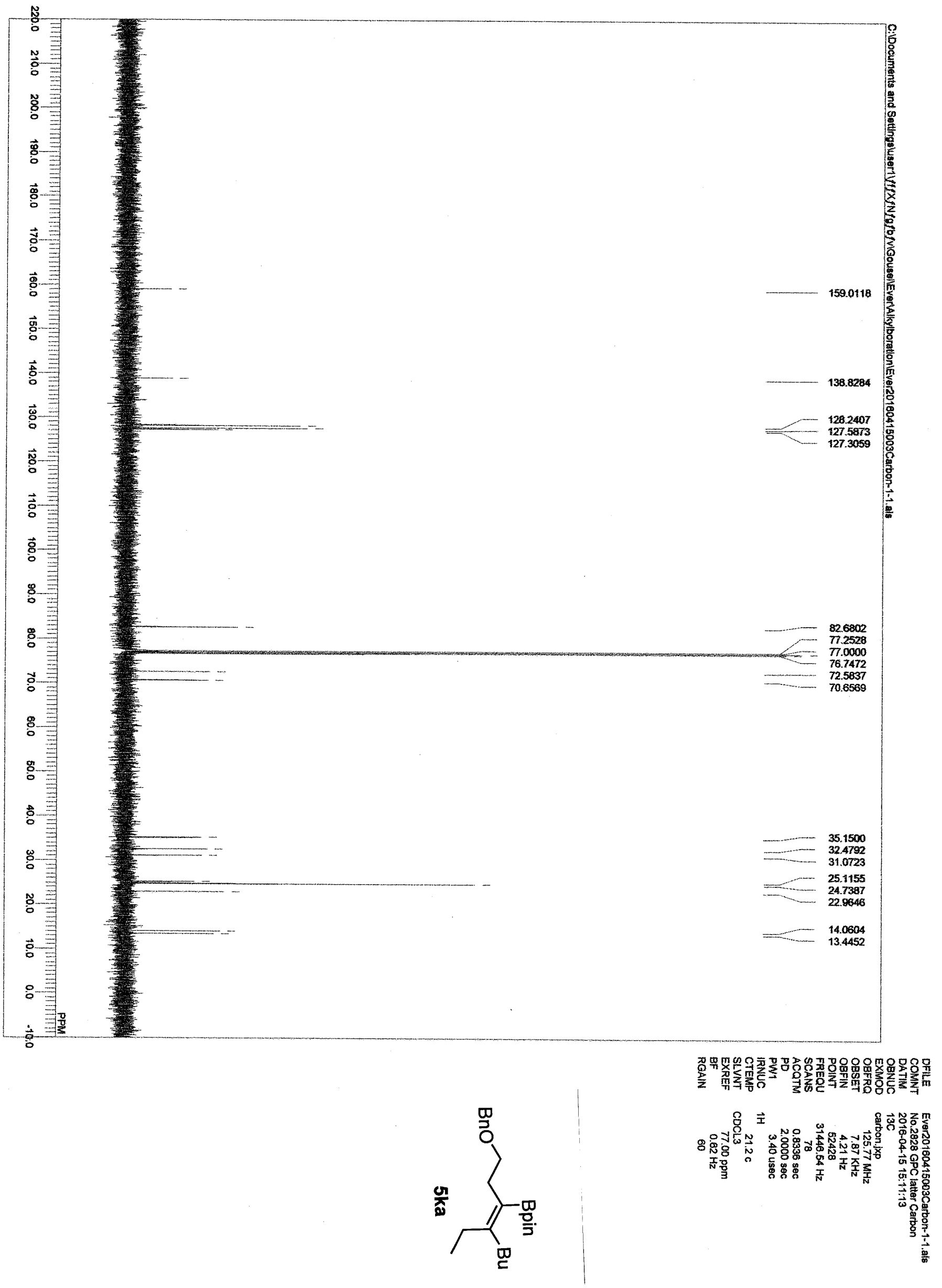




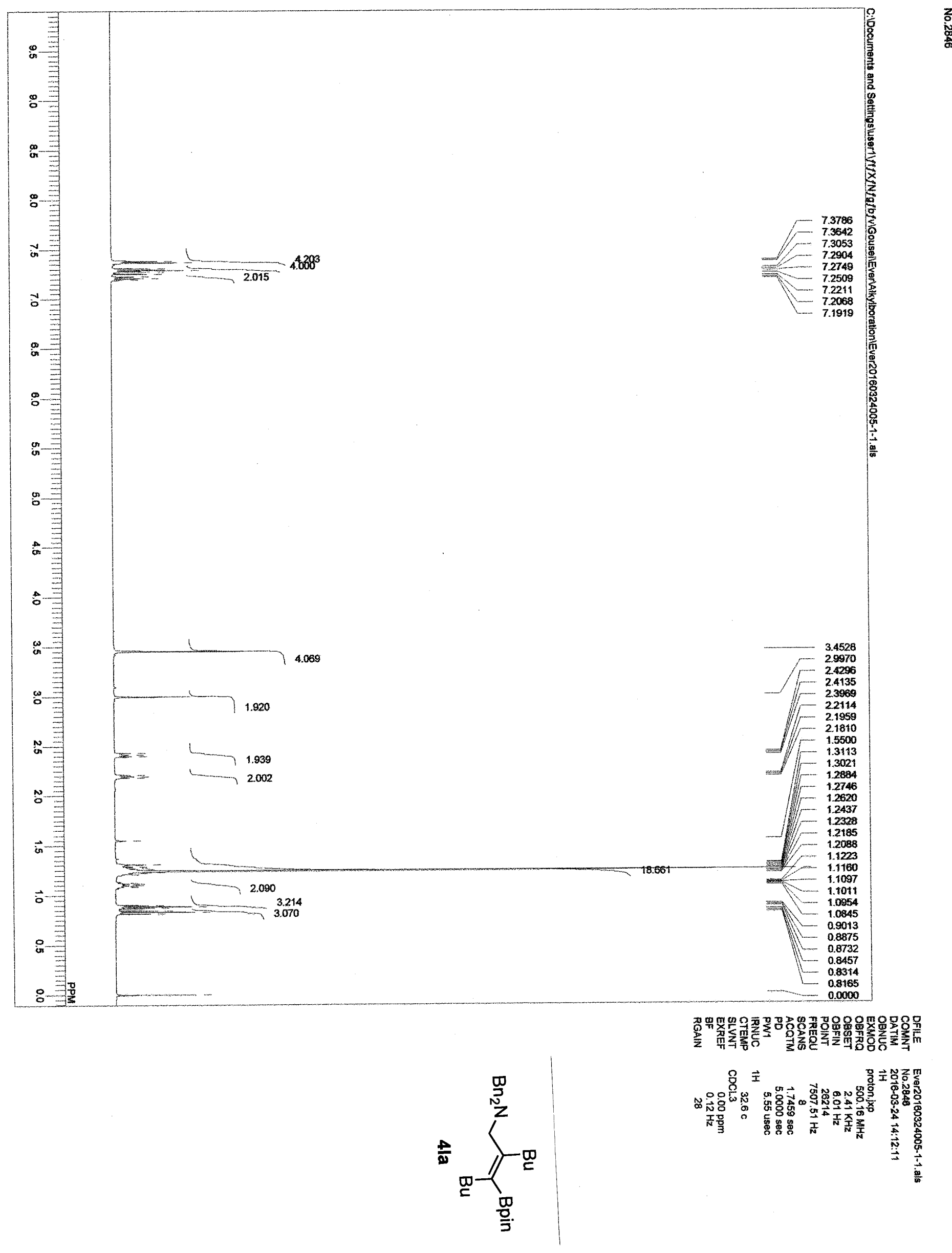




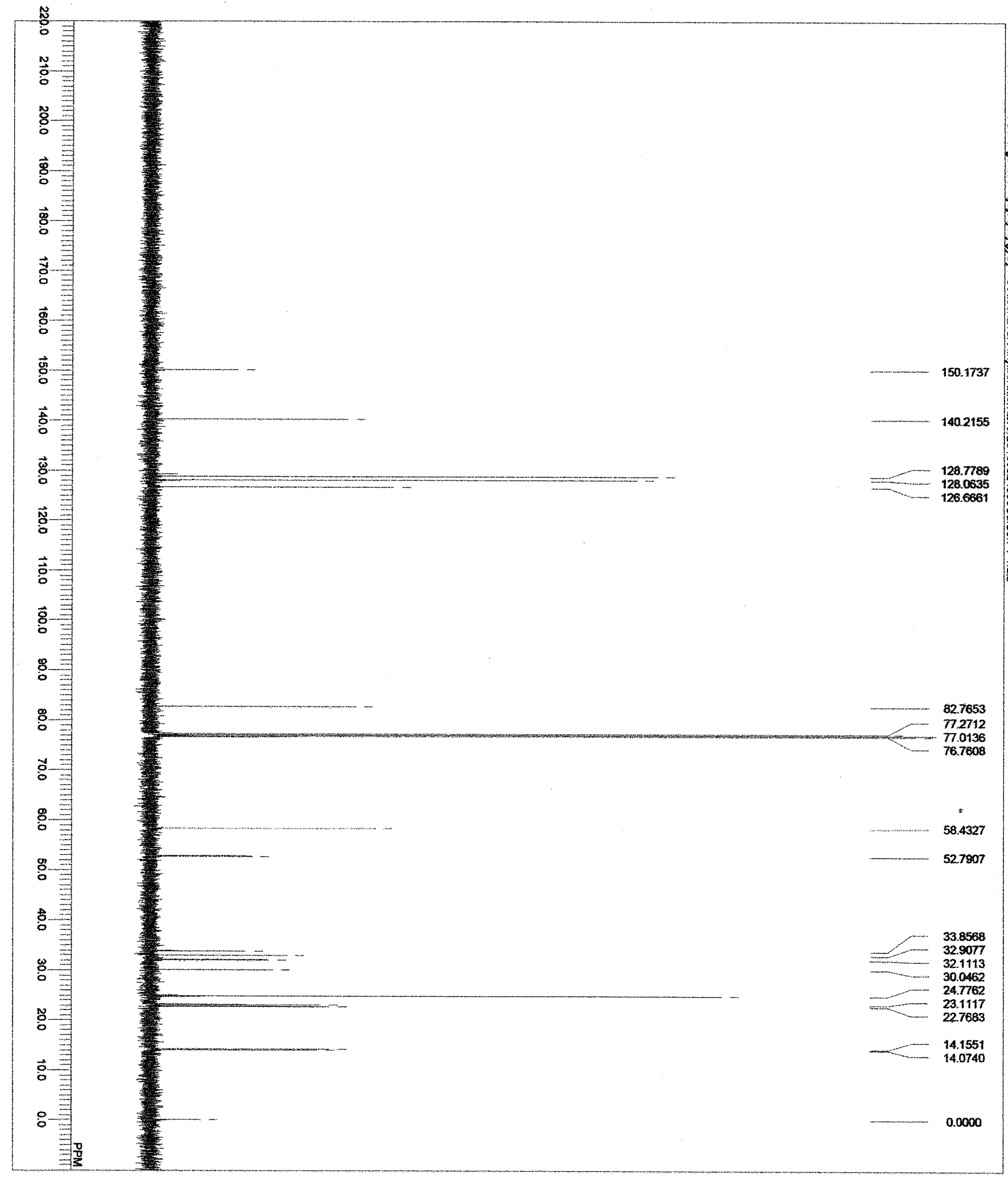

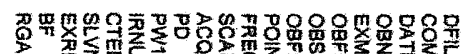

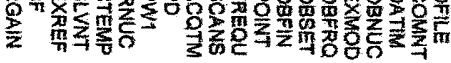

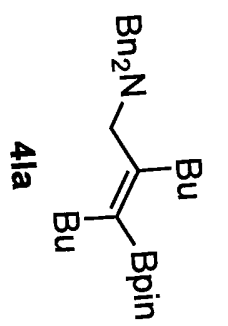

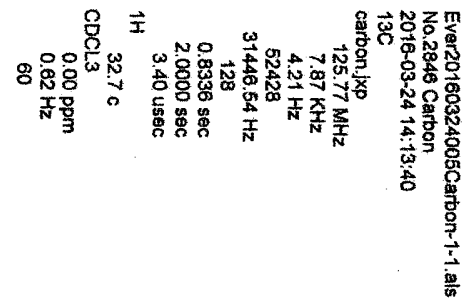




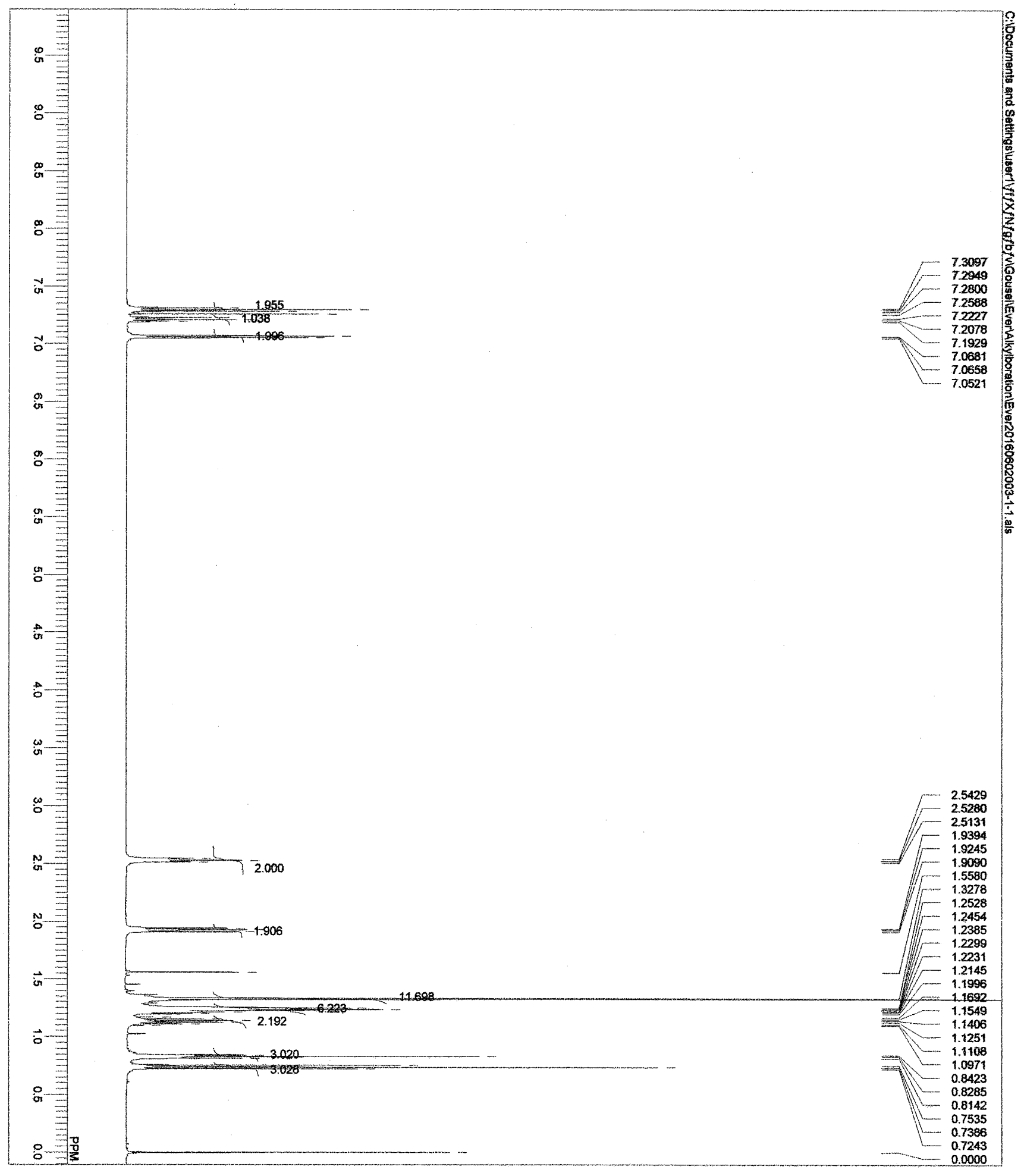




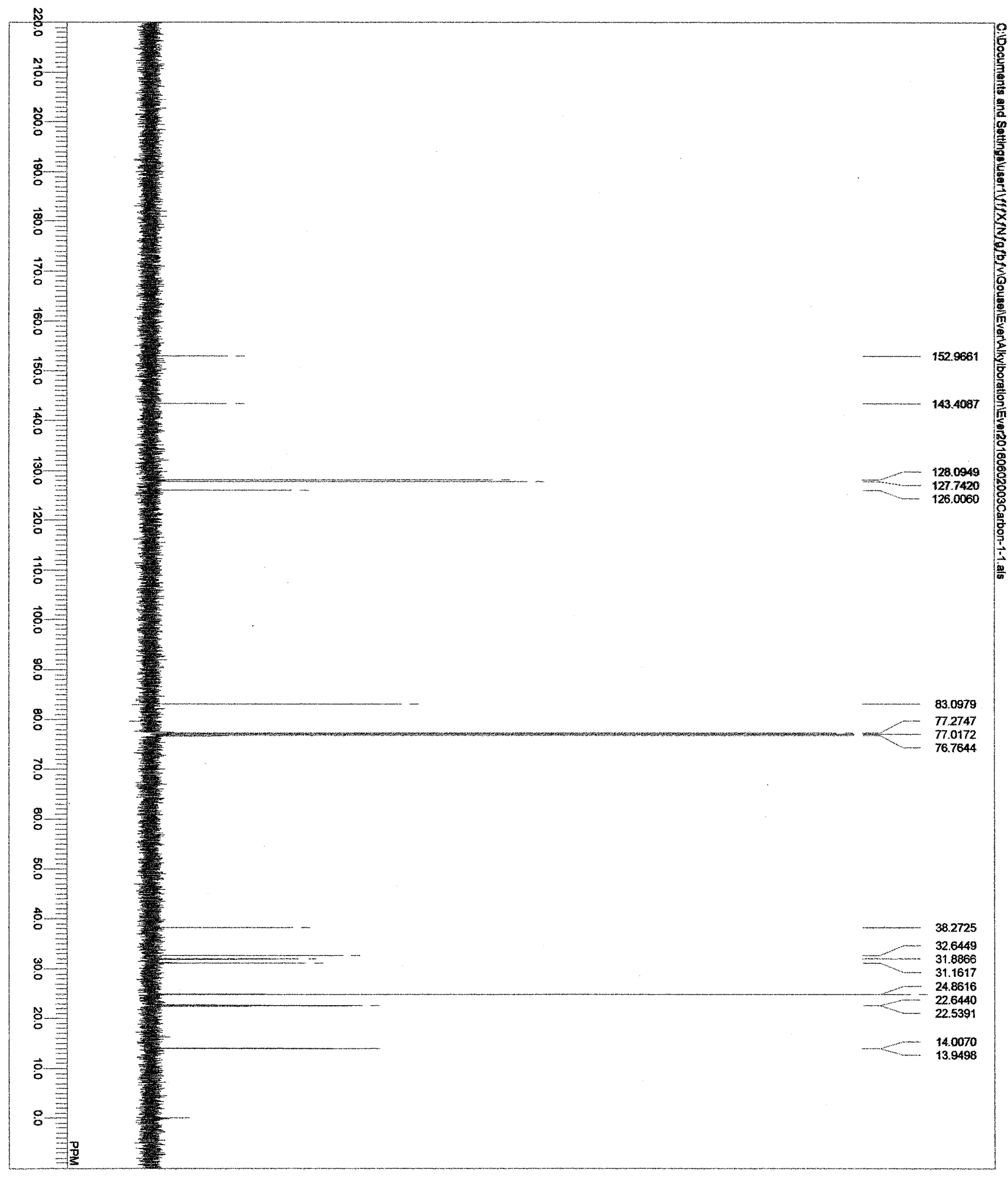

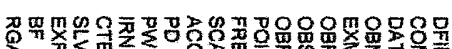

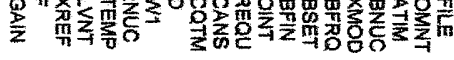

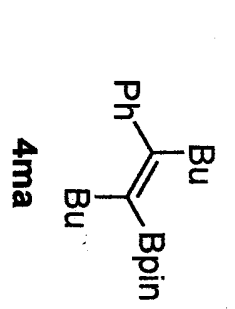

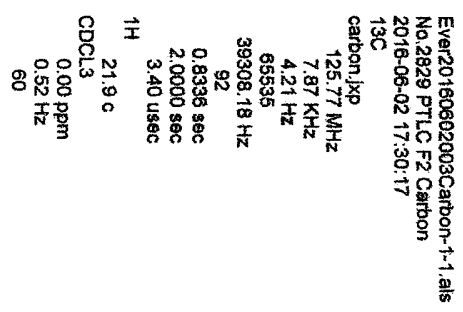




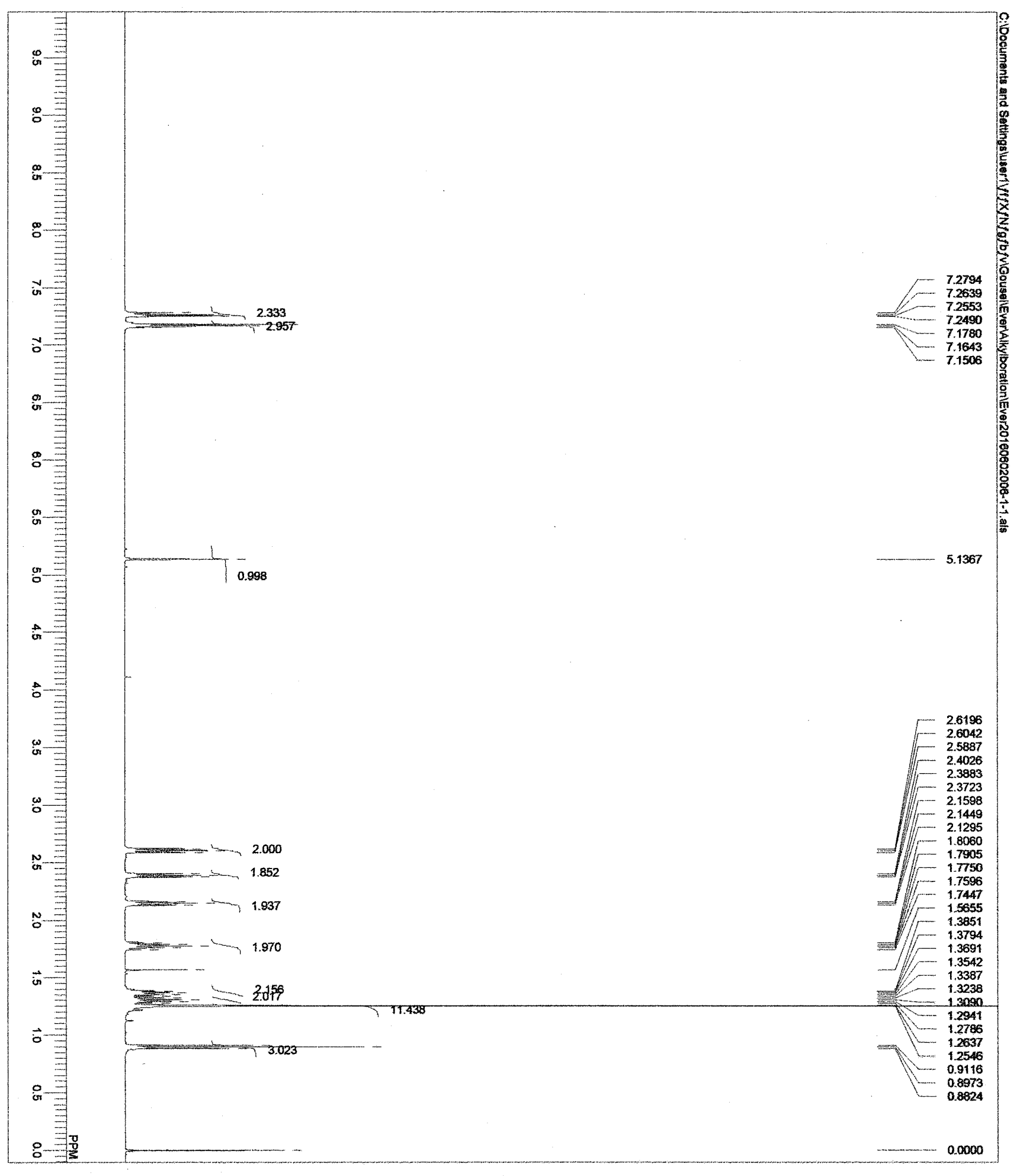

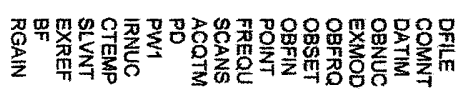

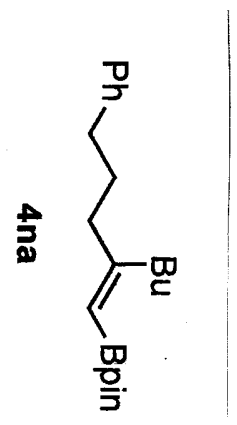

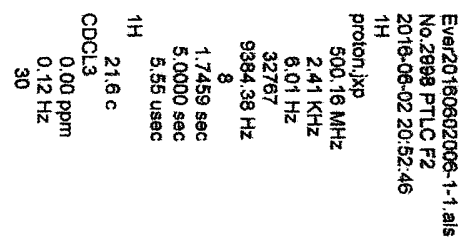




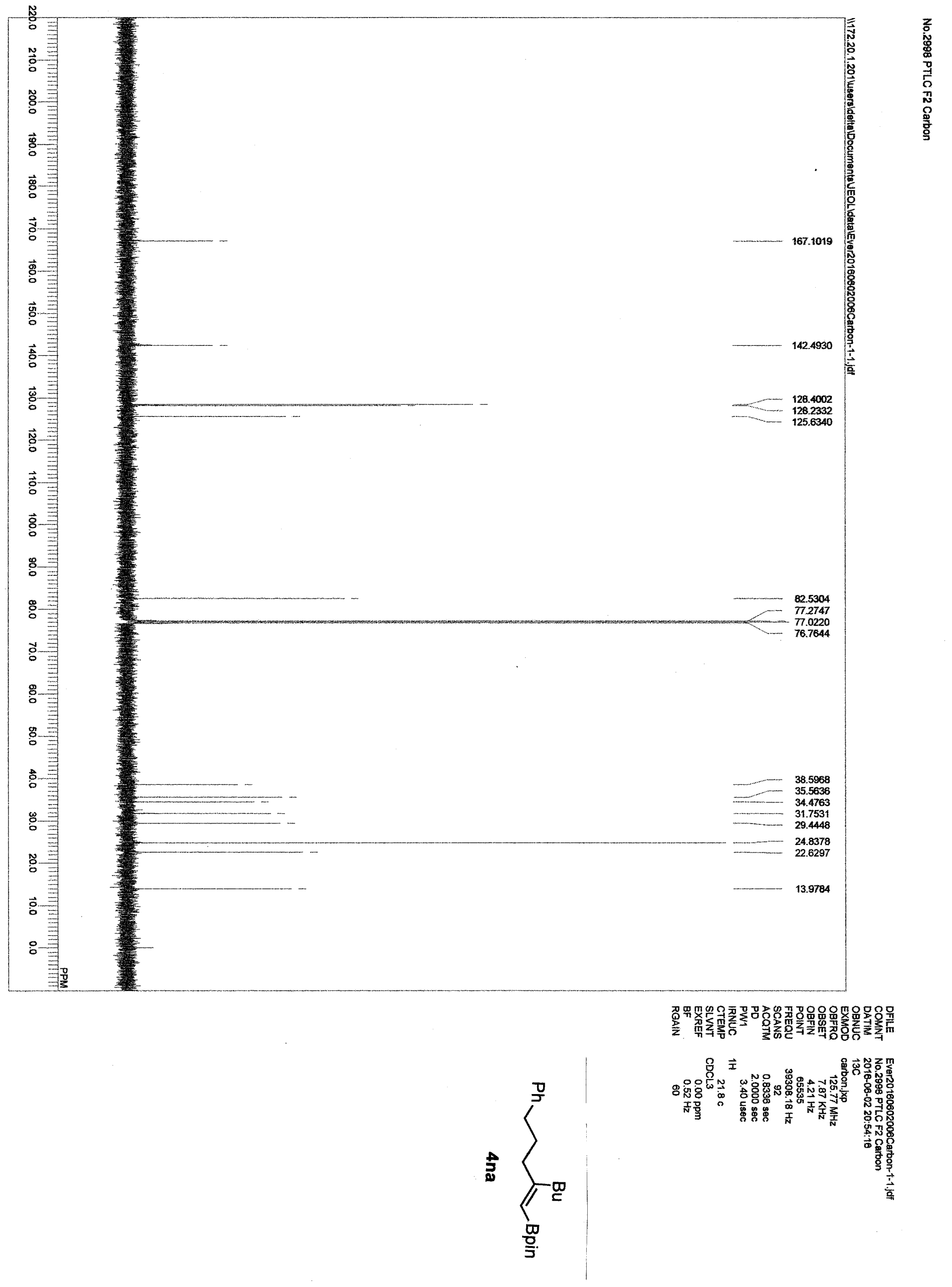




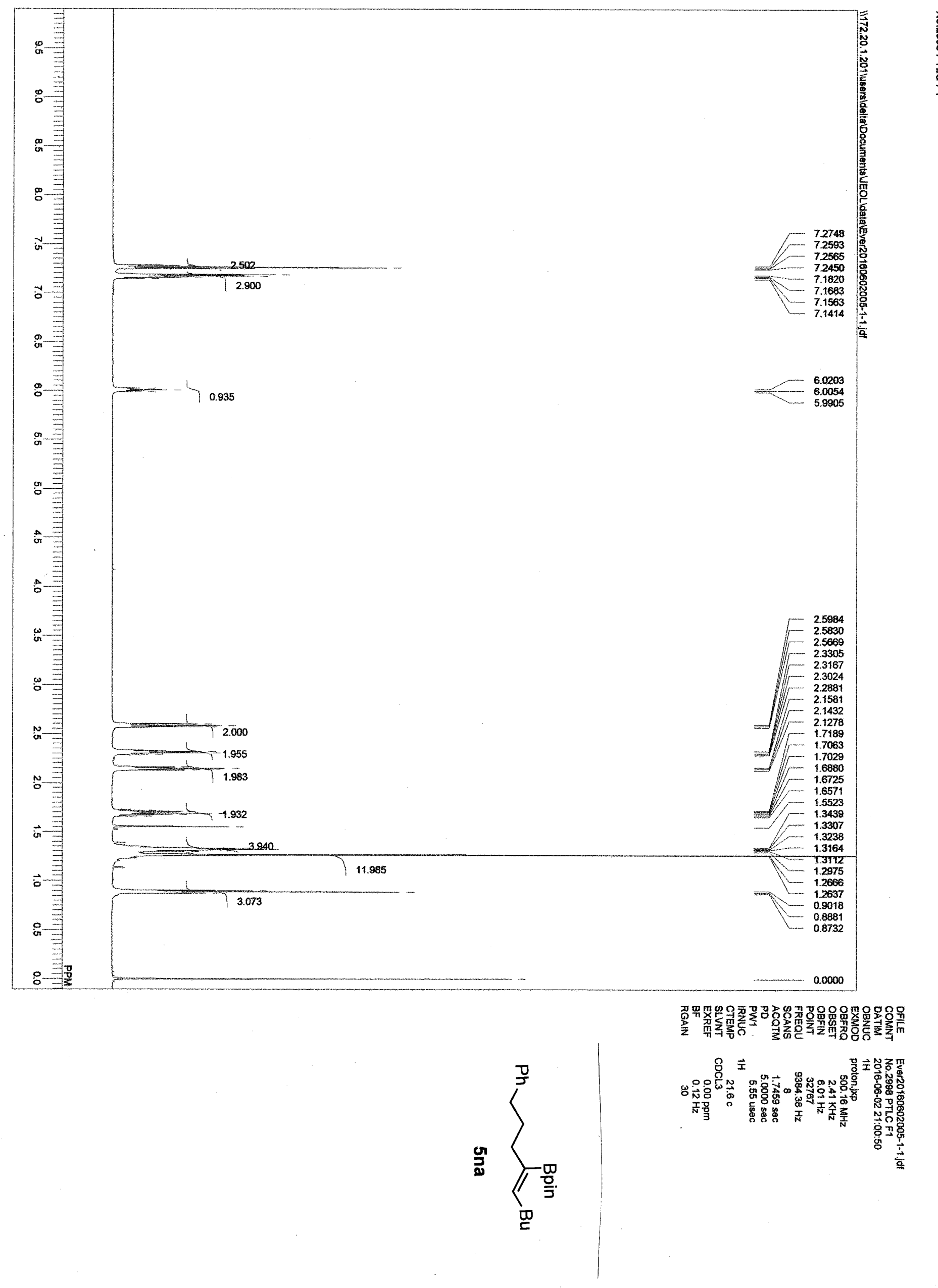




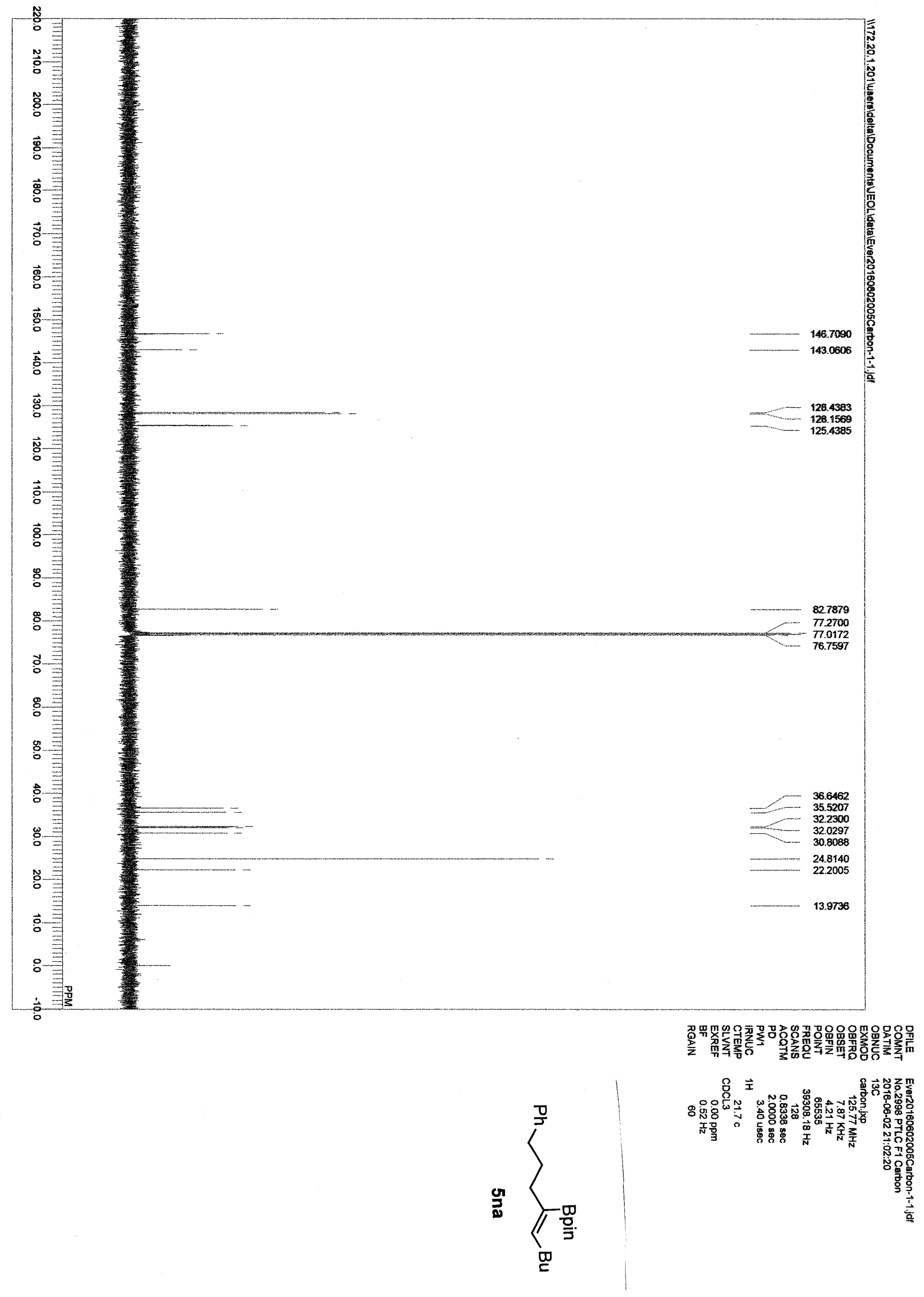




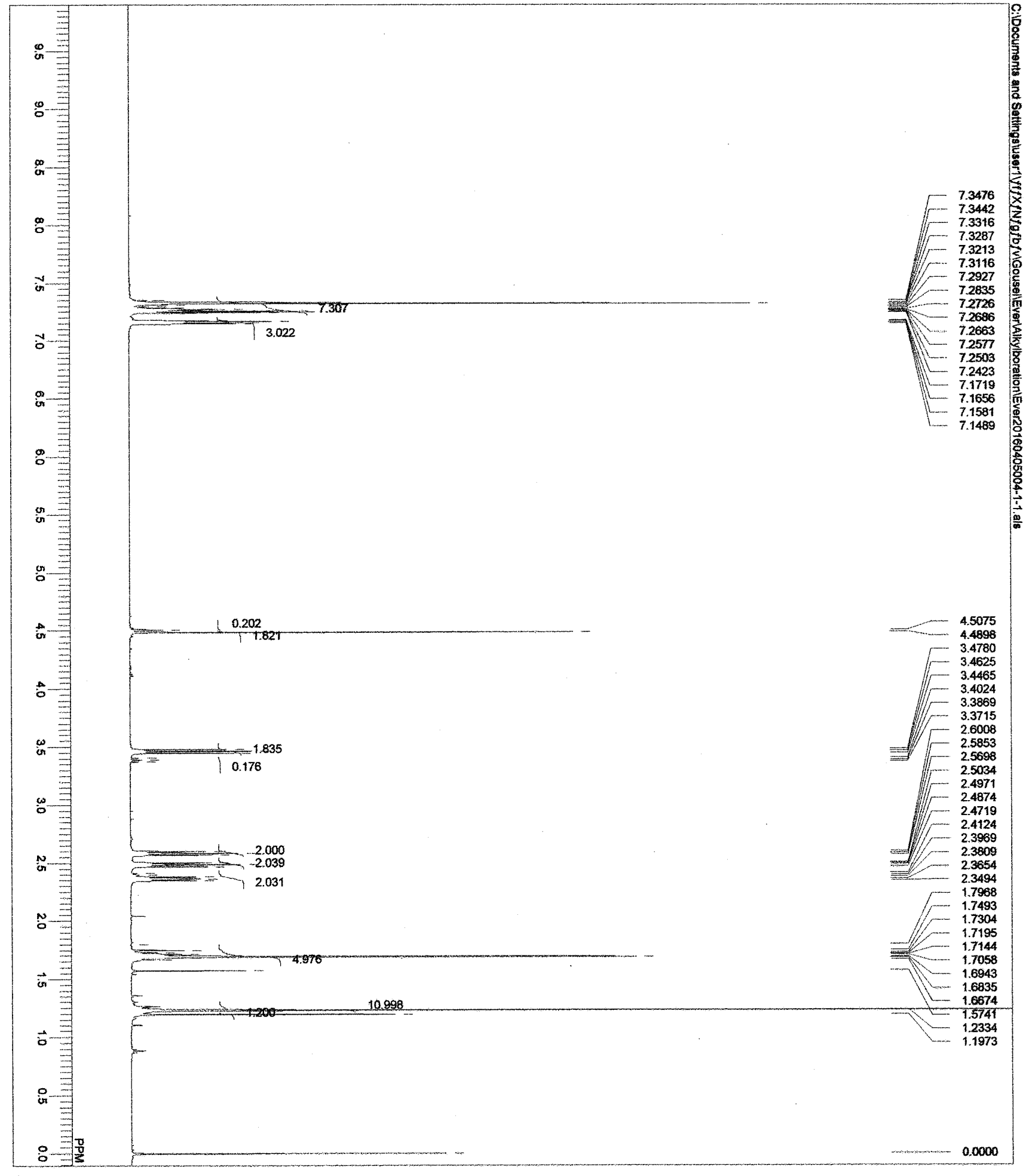




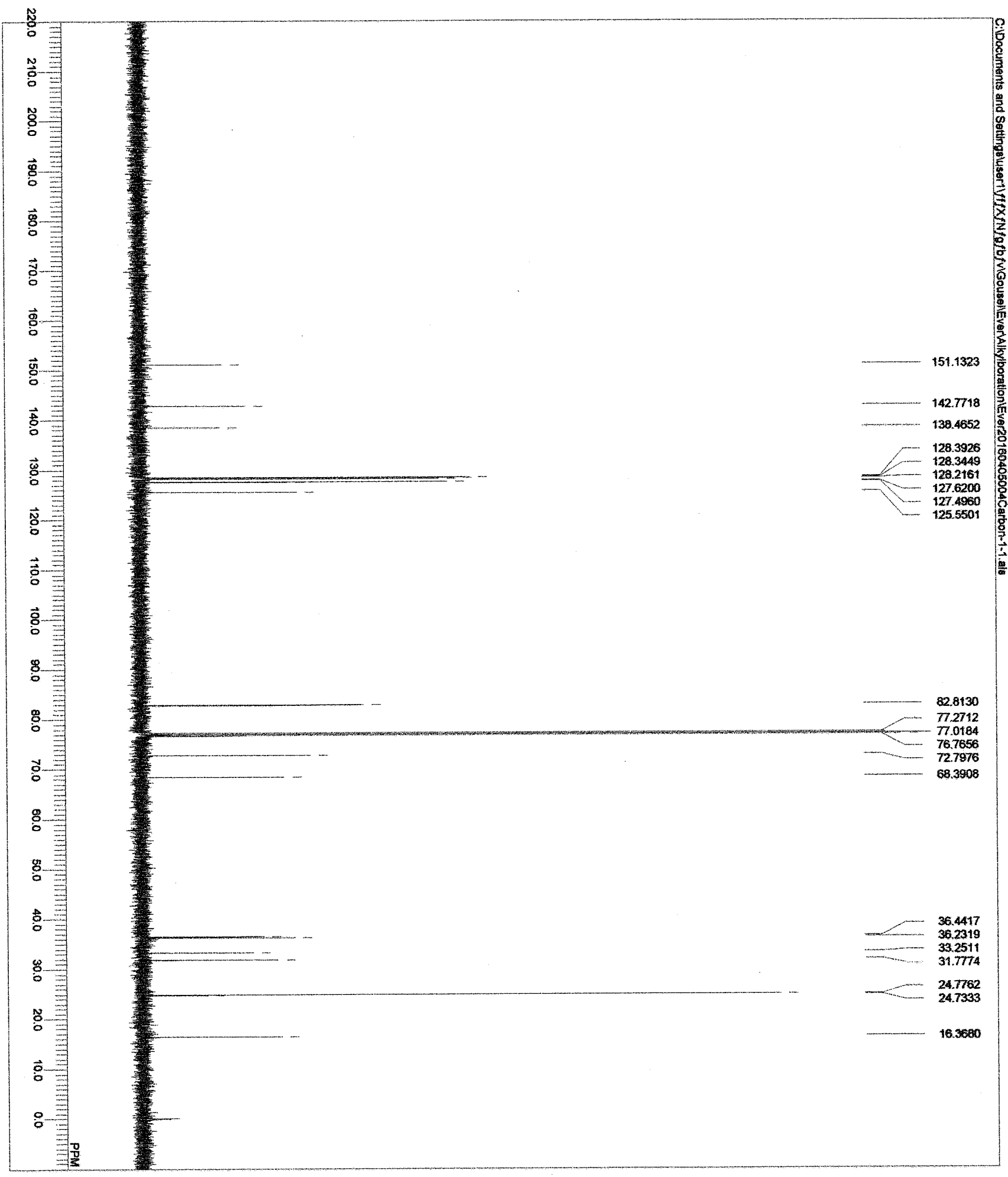

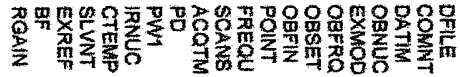

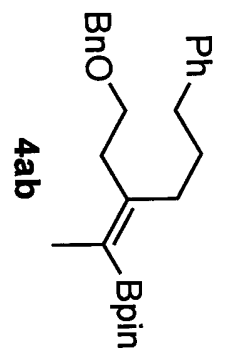

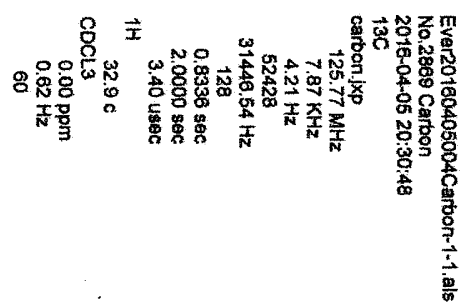




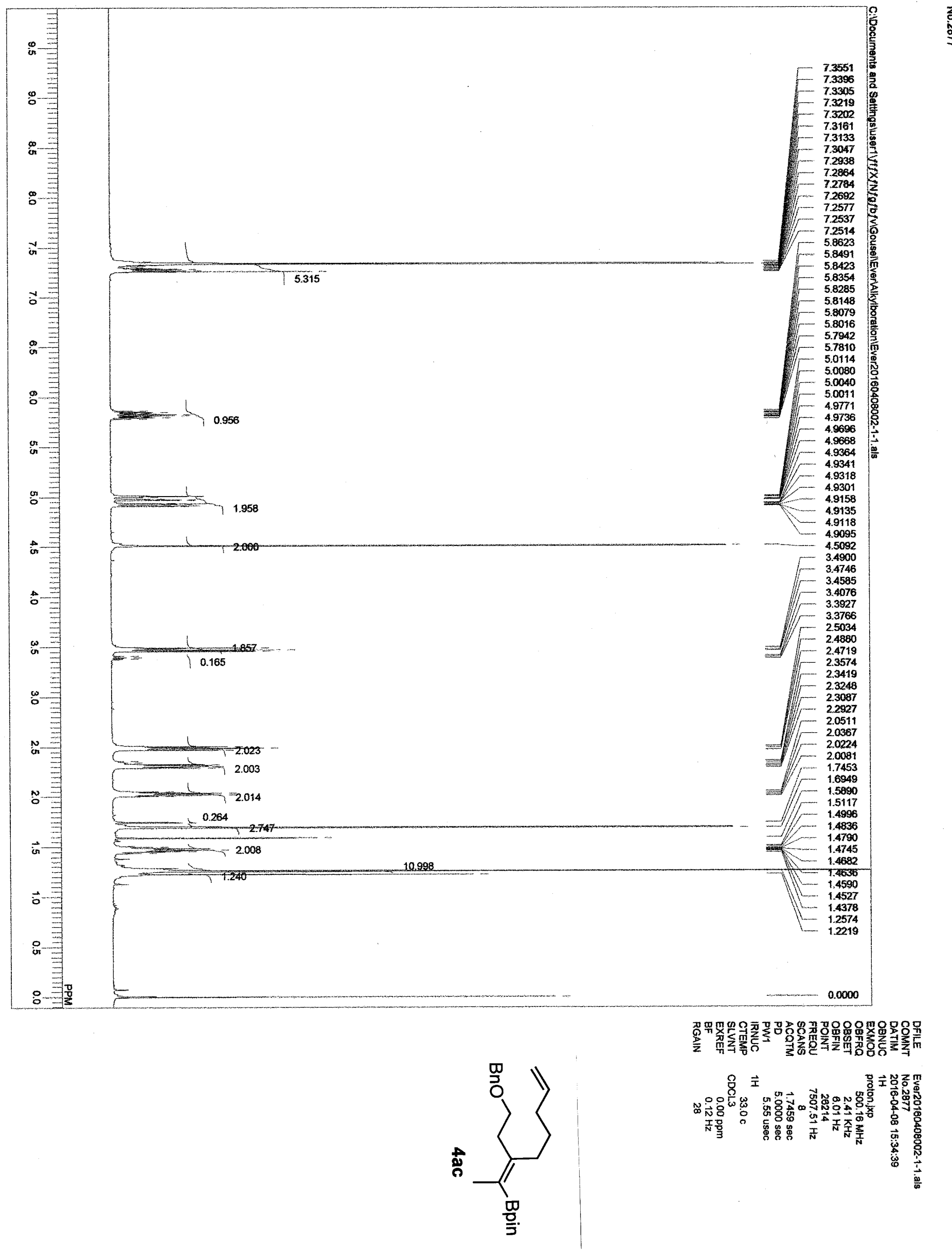




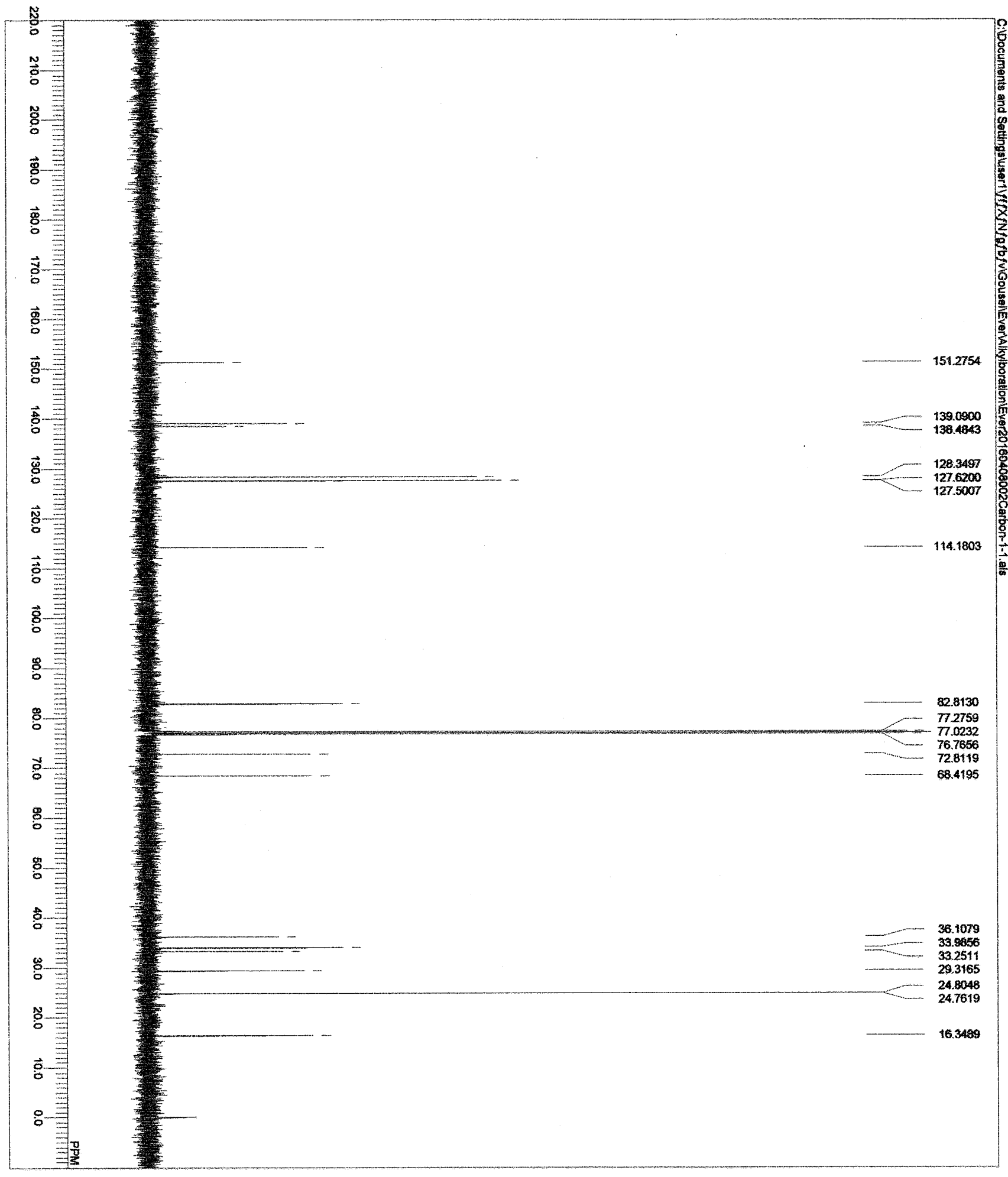




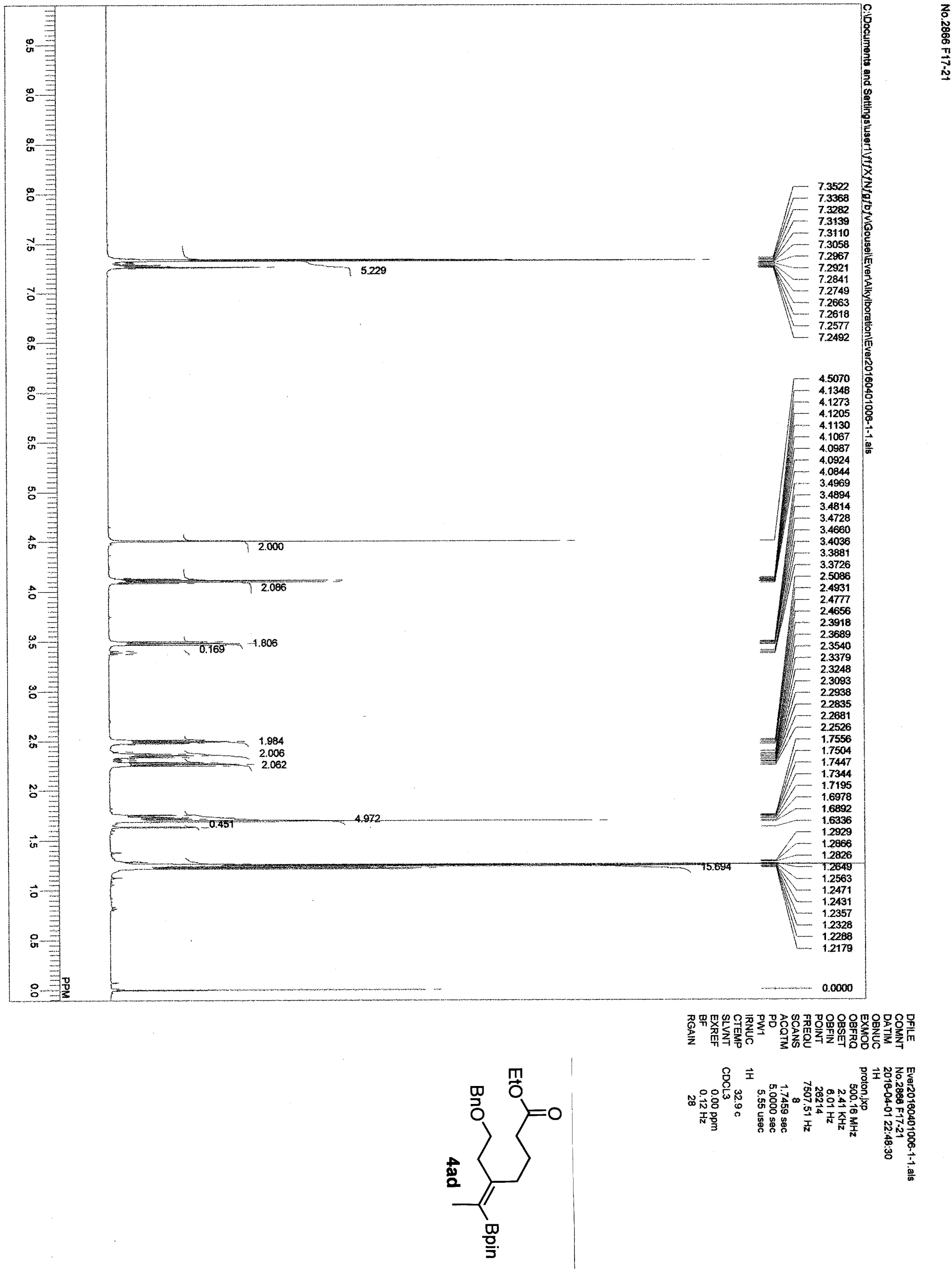



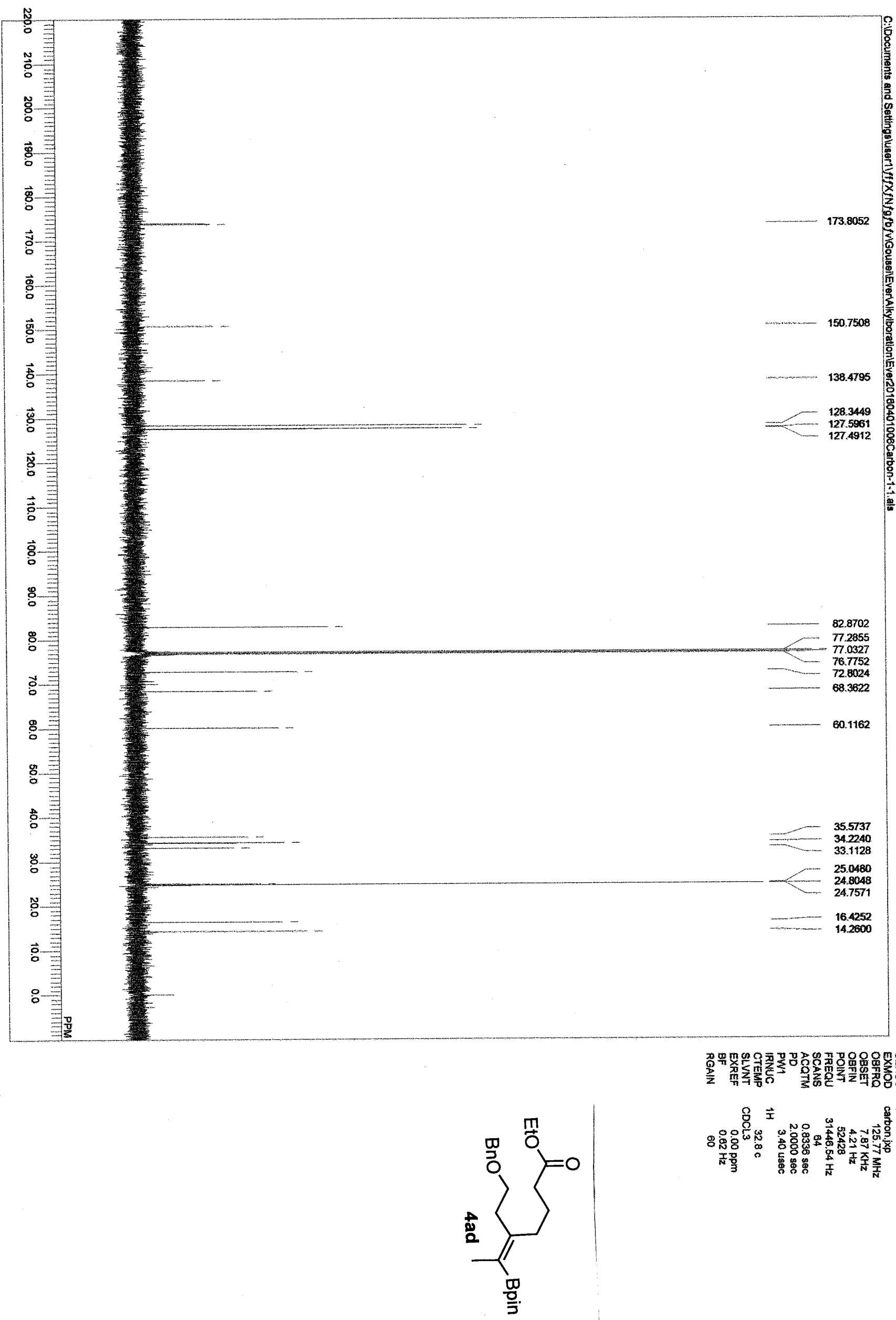

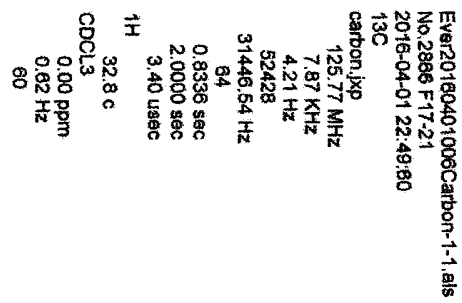




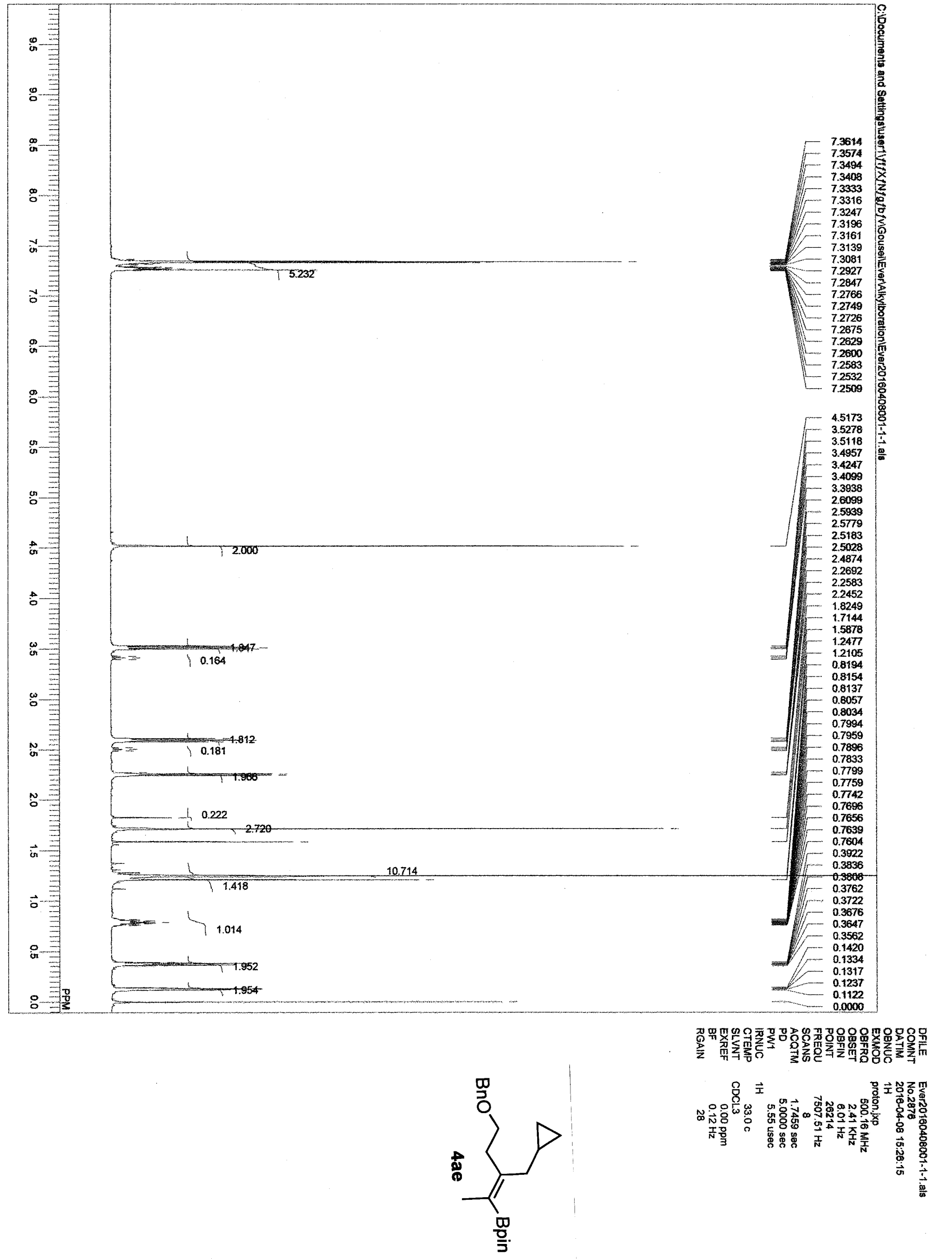




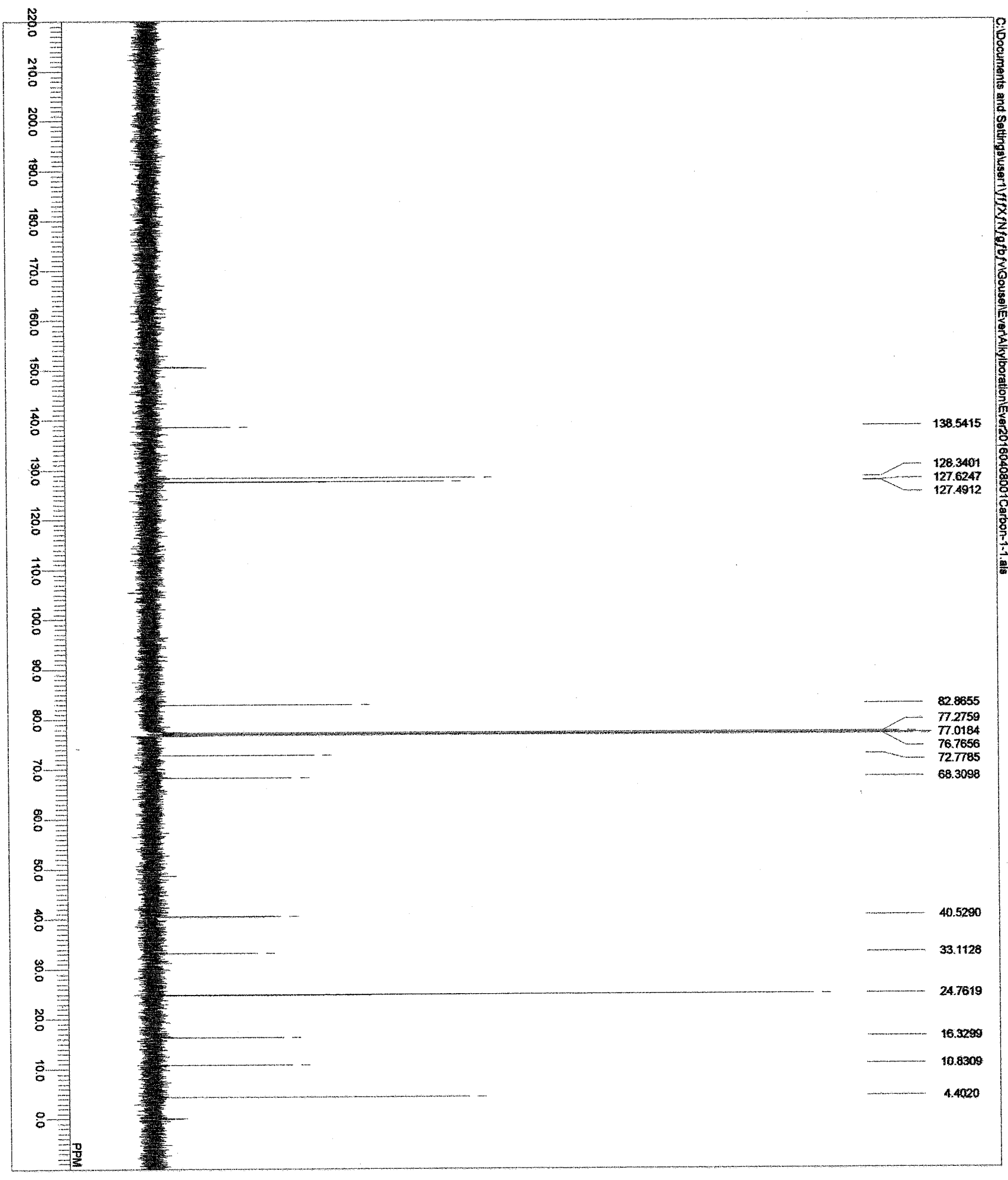

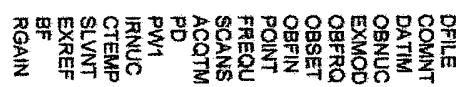

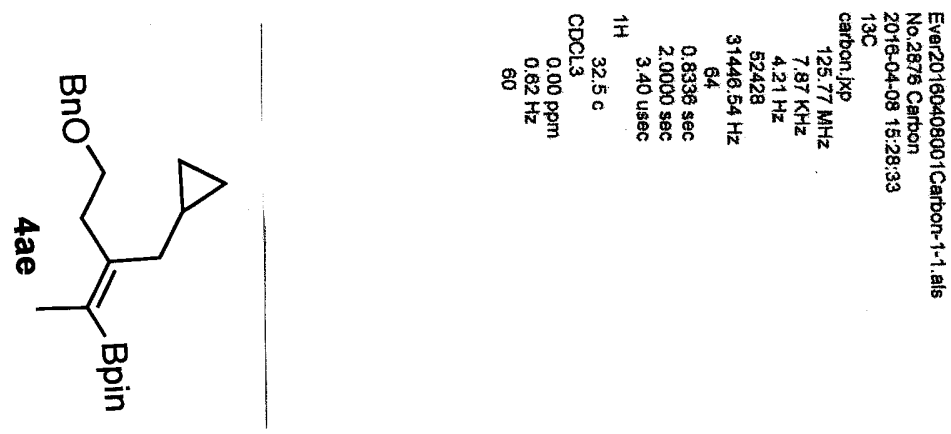




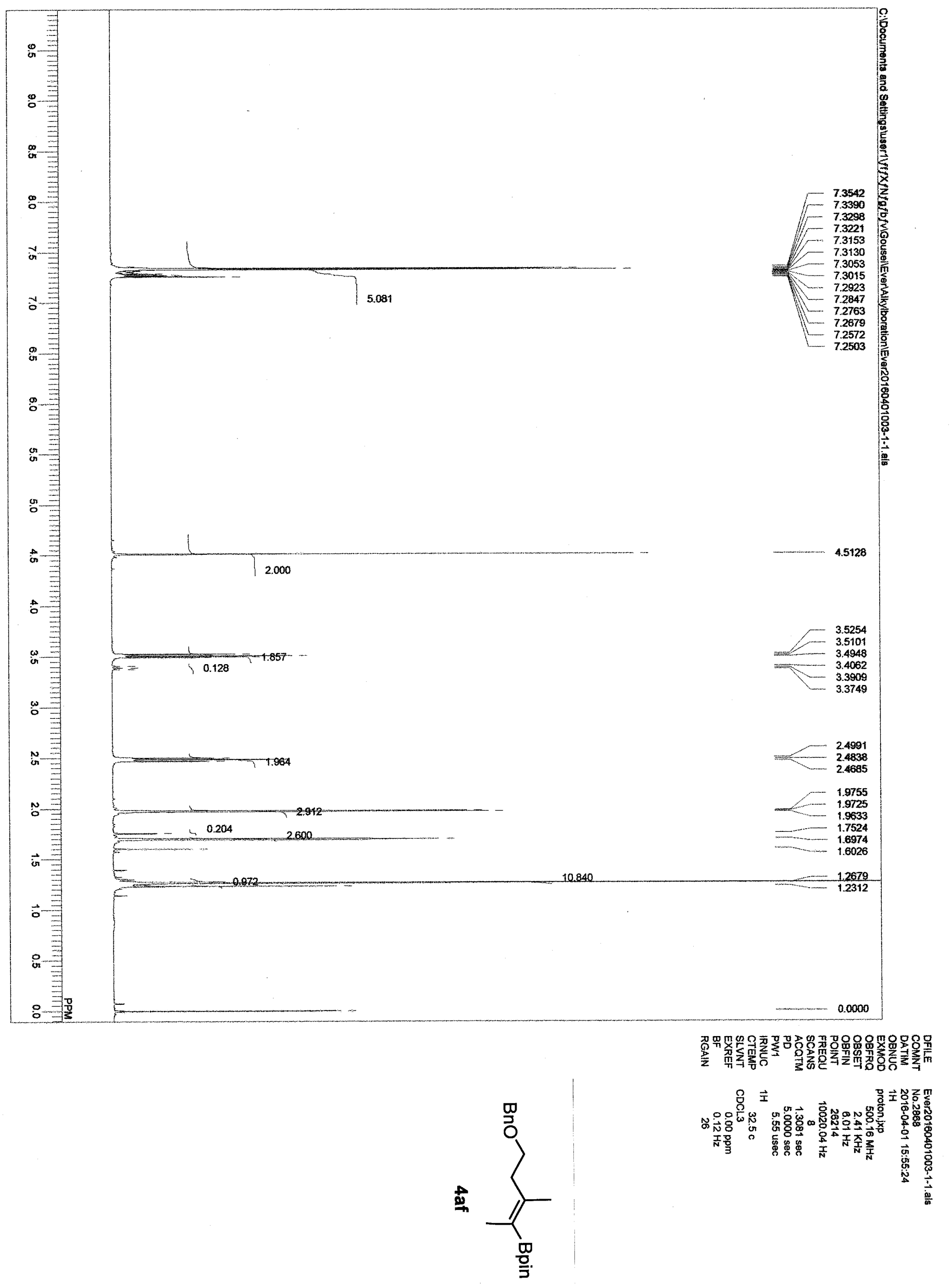




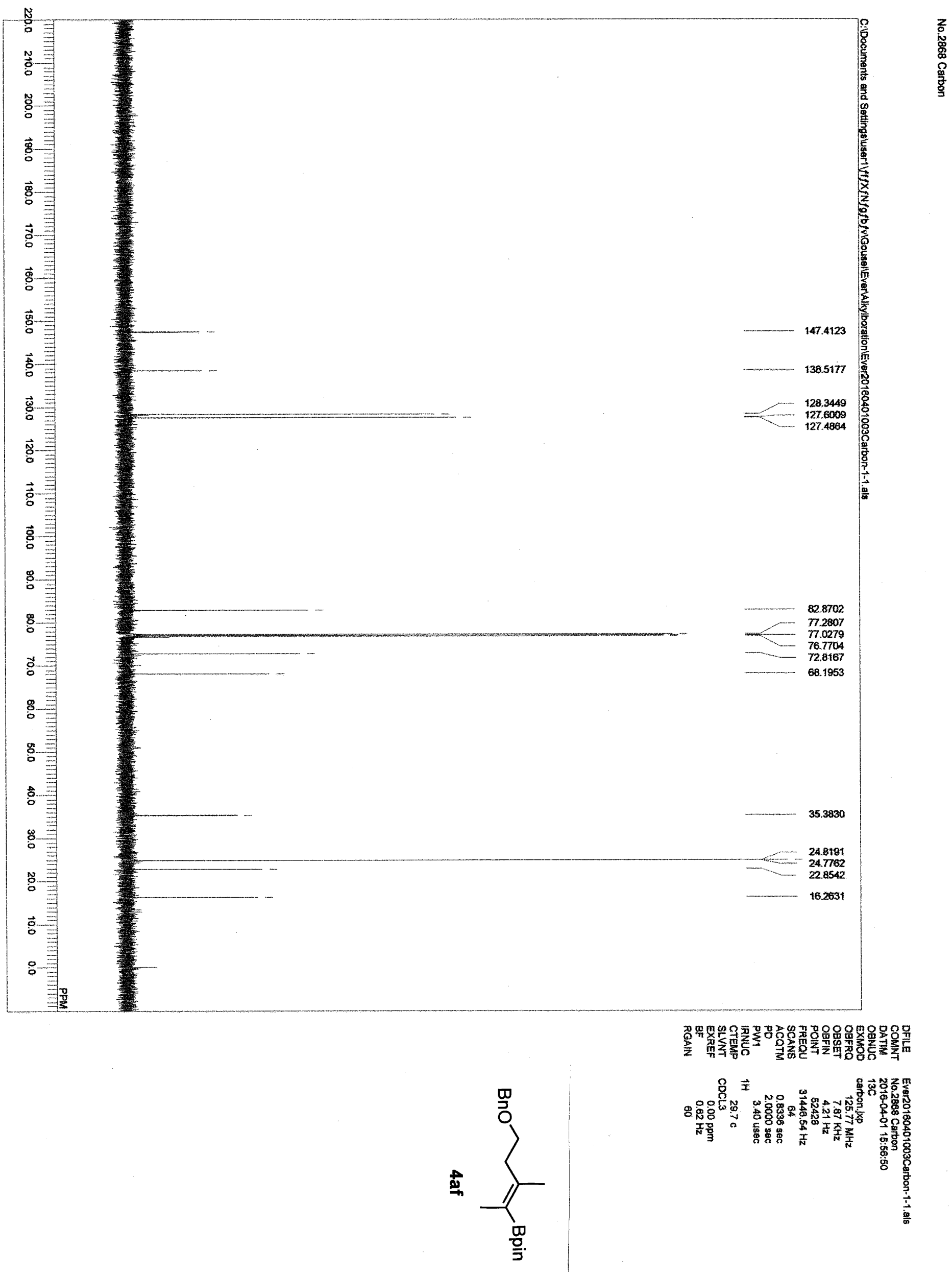




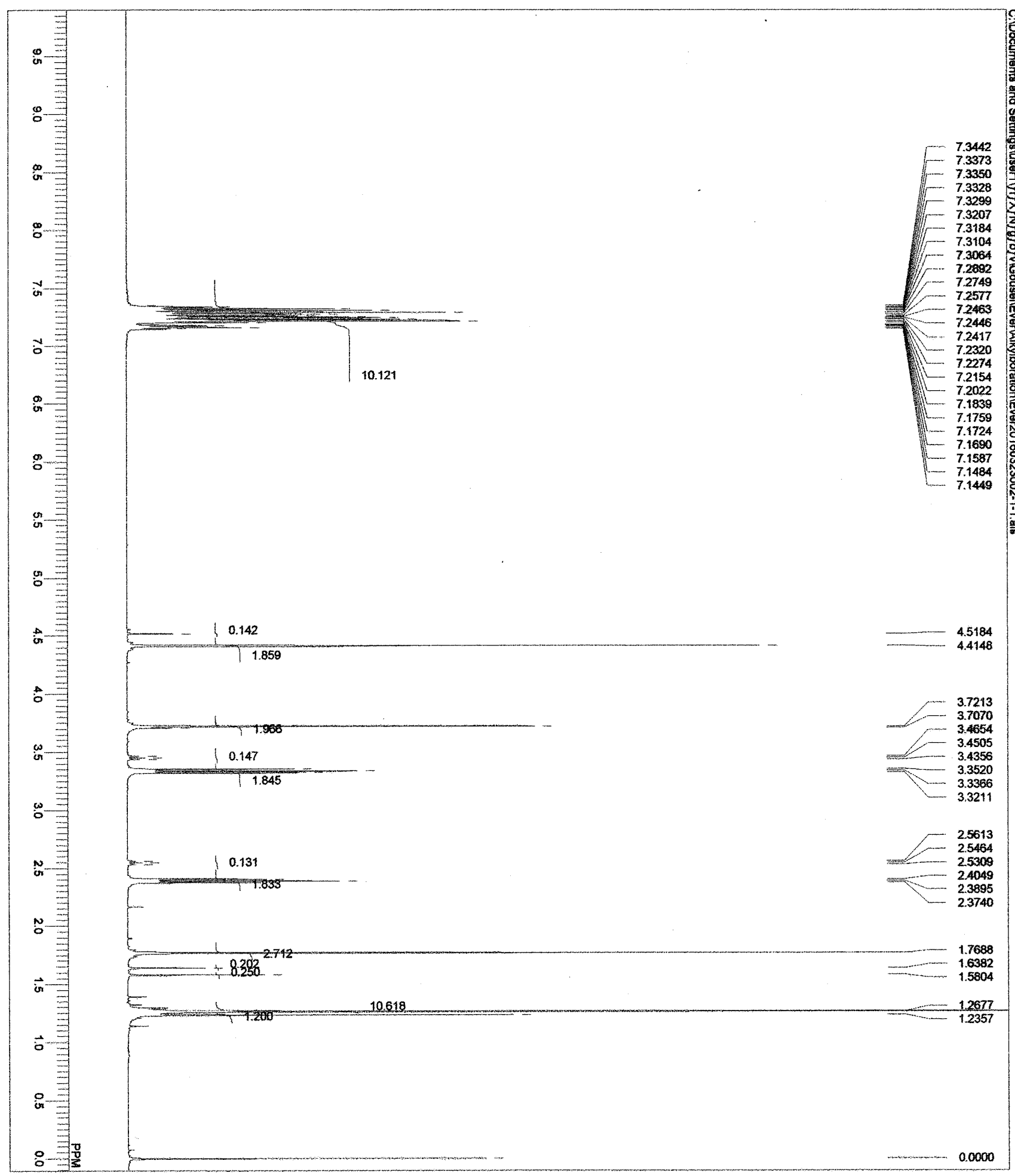

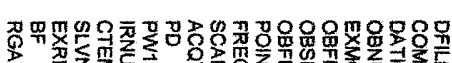

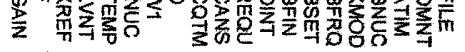<smiles>CC(C)=C(C)CC[O]</smiles> 


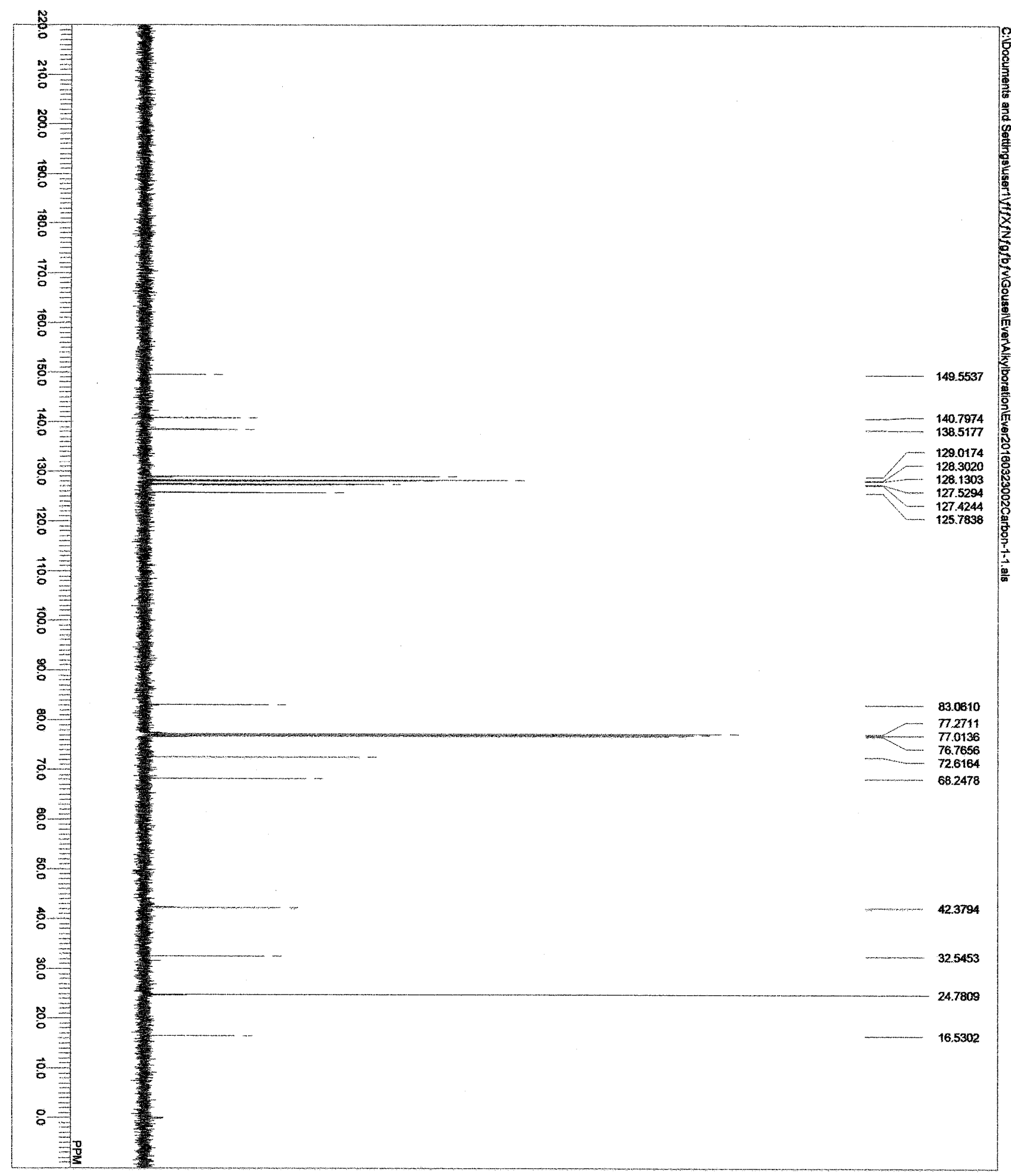

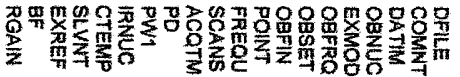
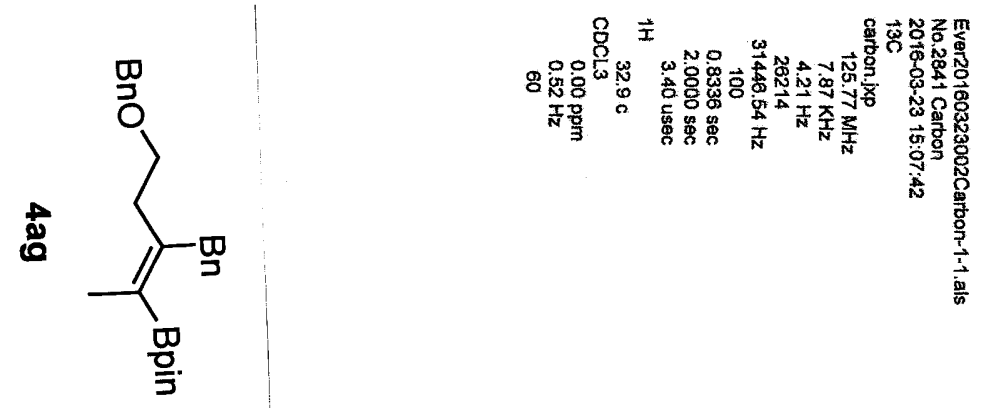


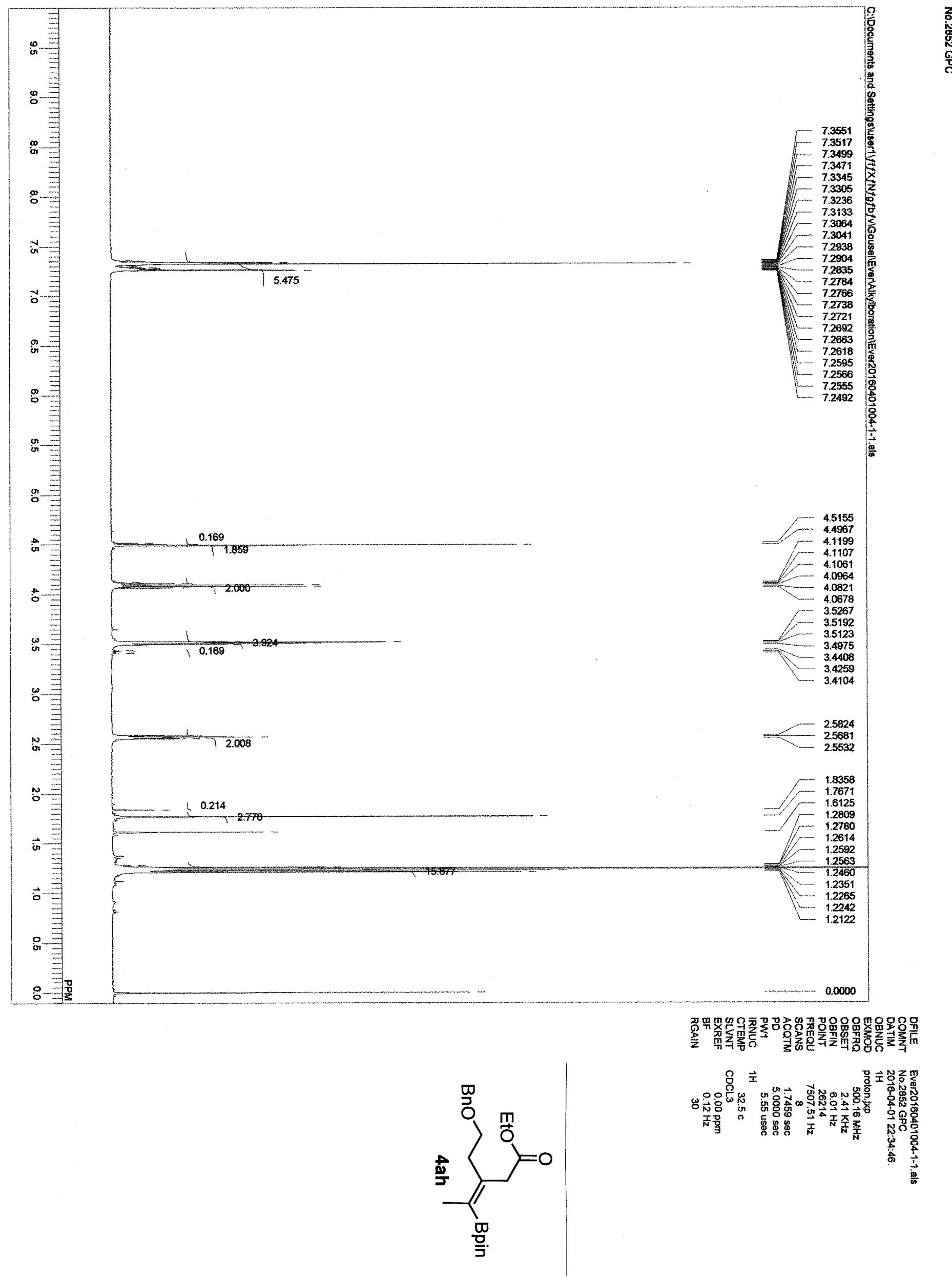




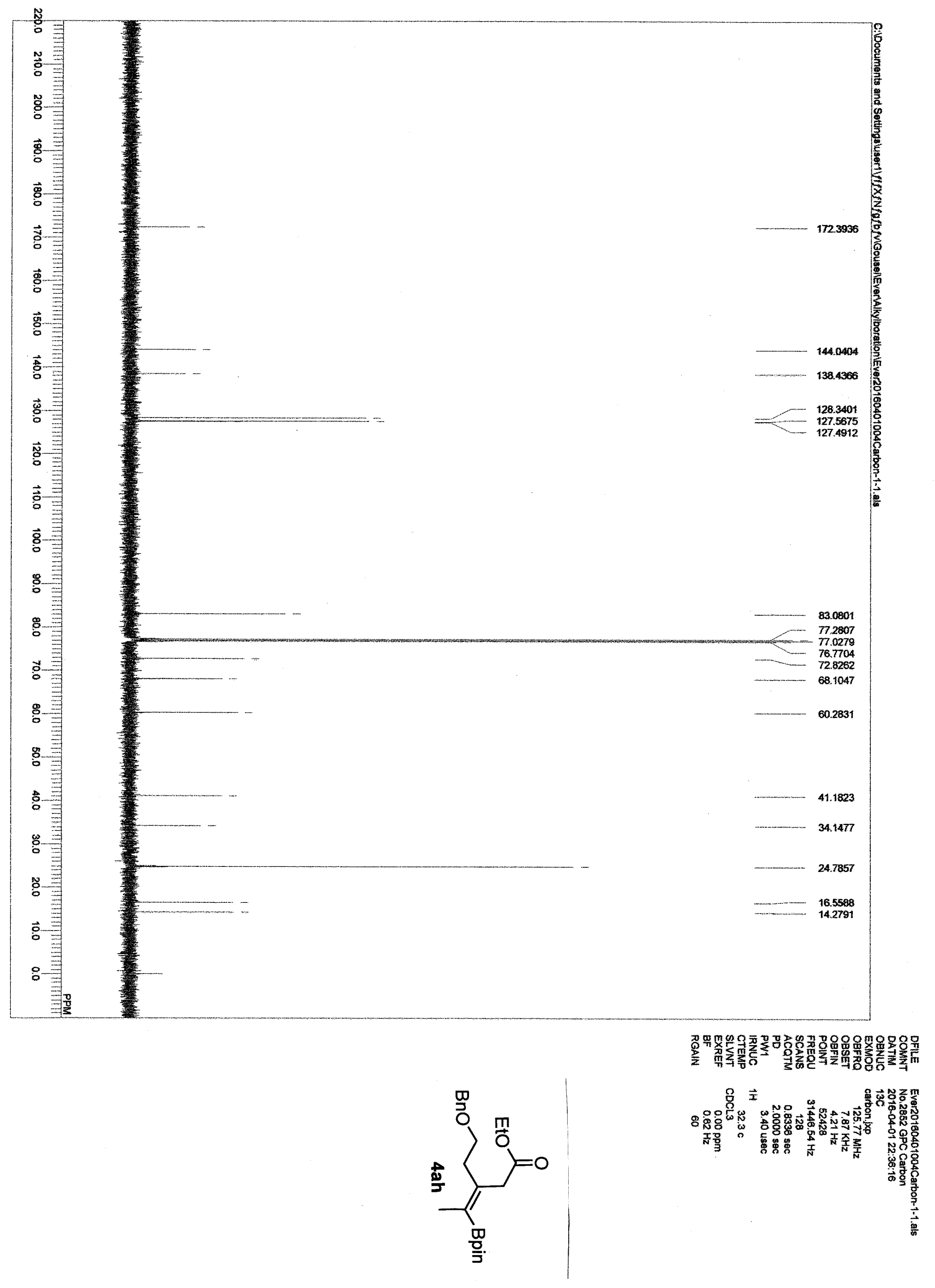




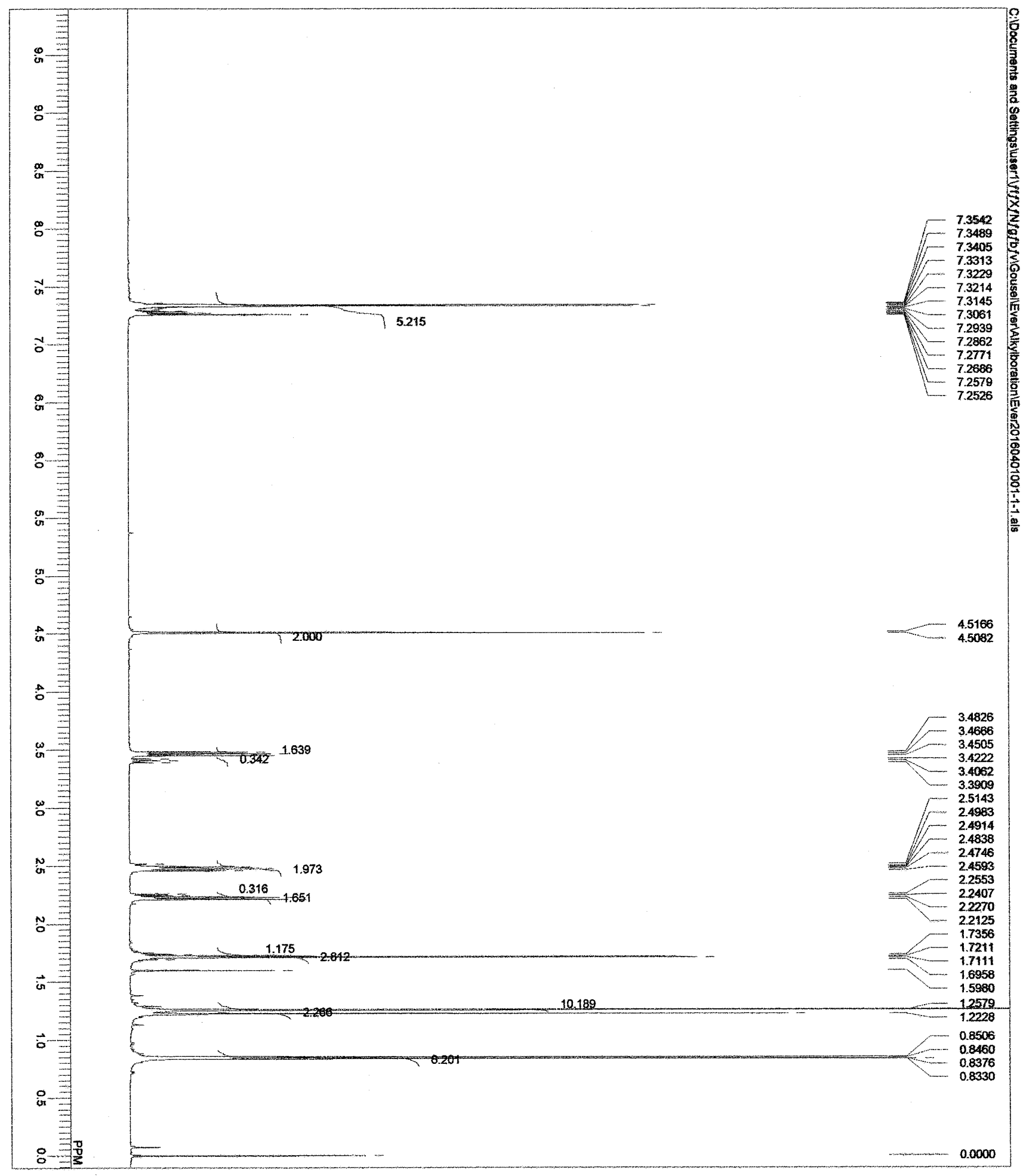

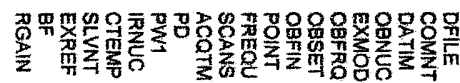

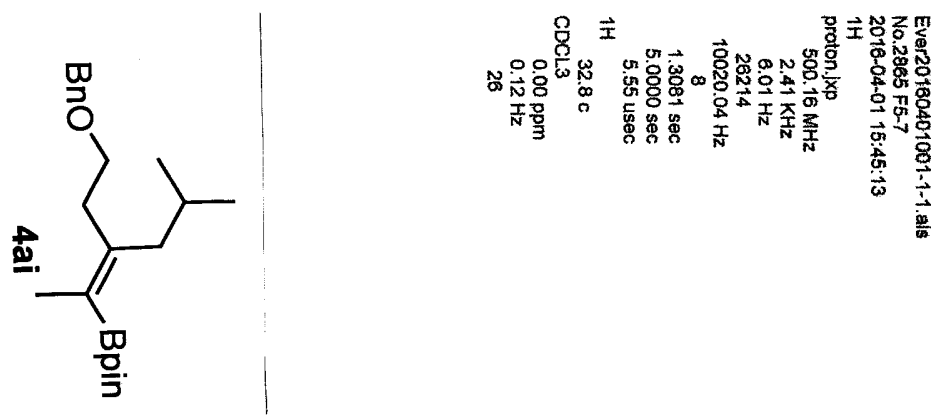




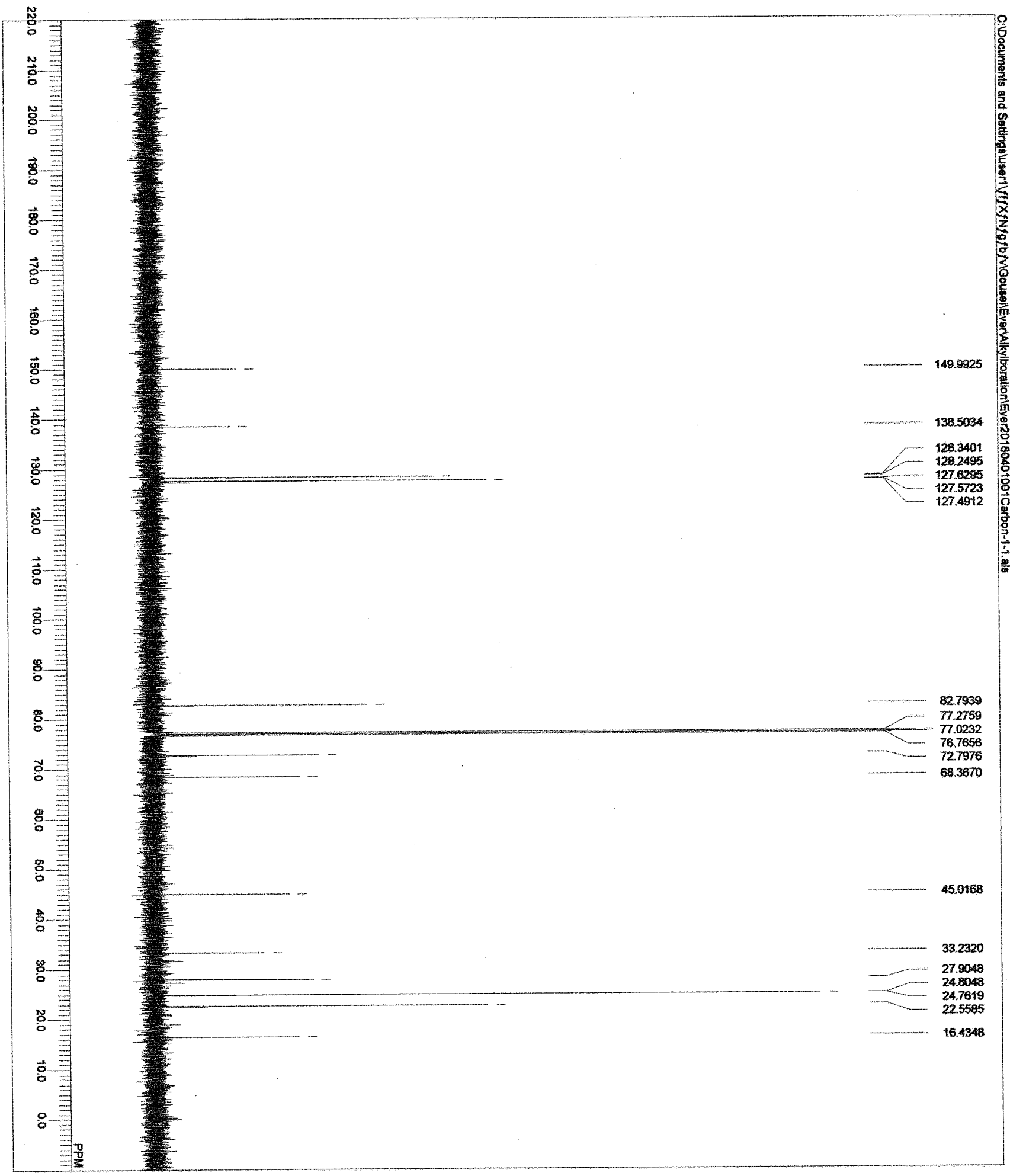

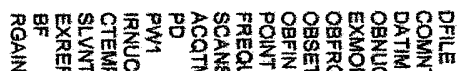

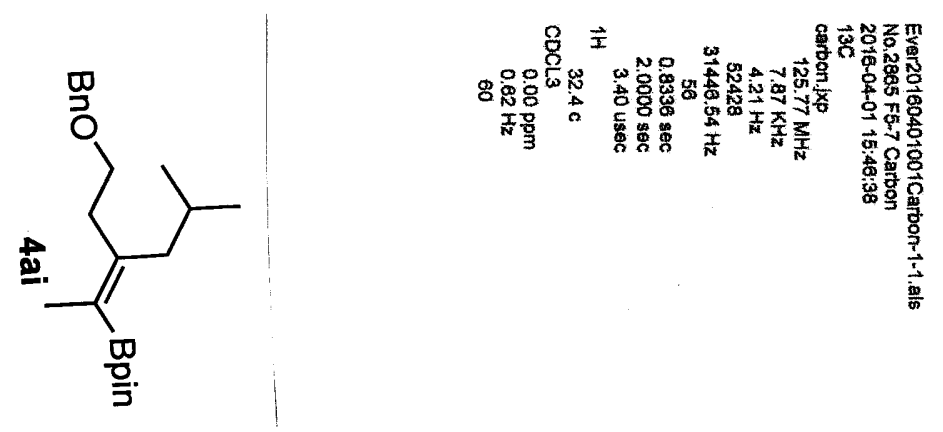




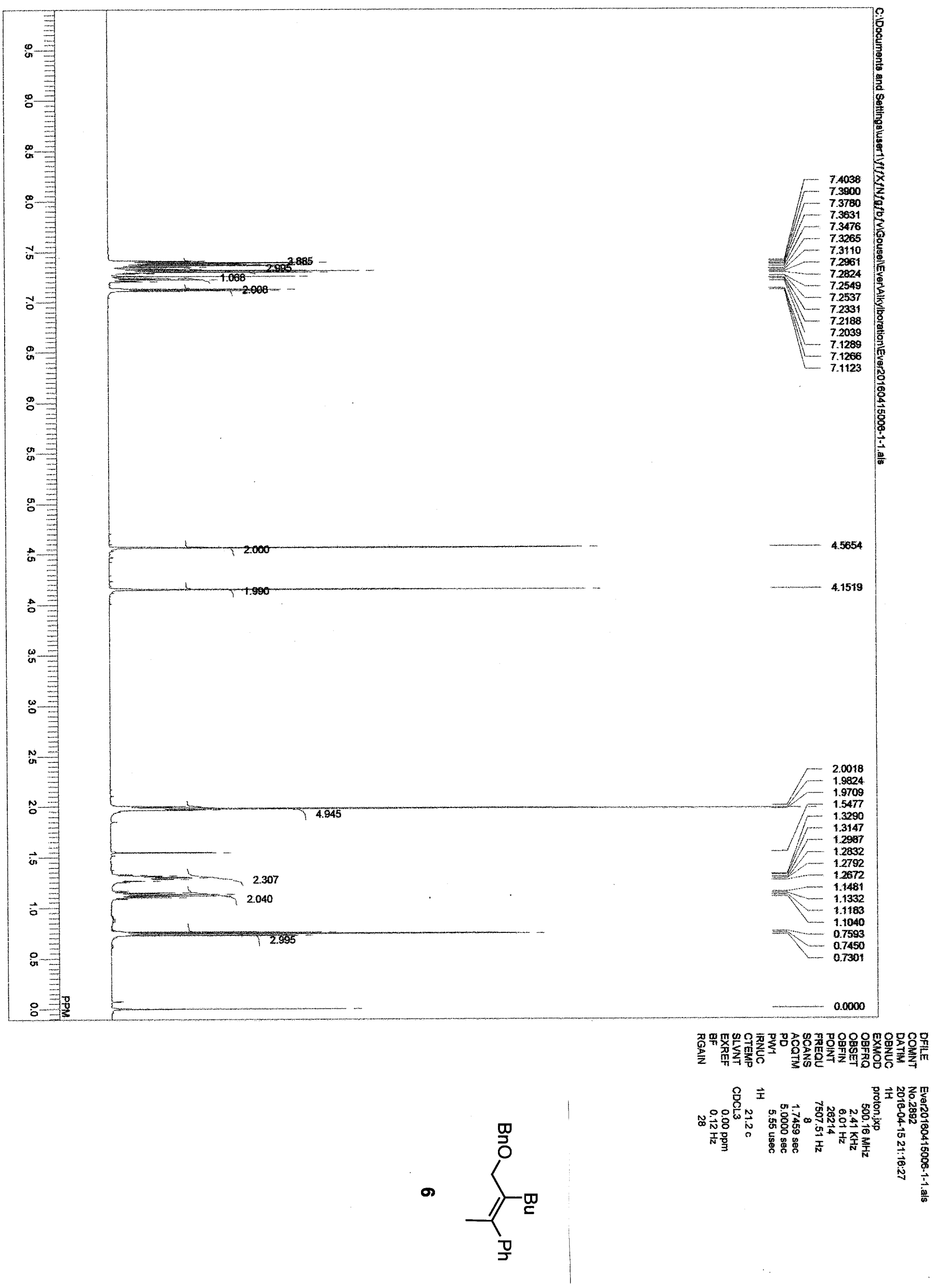




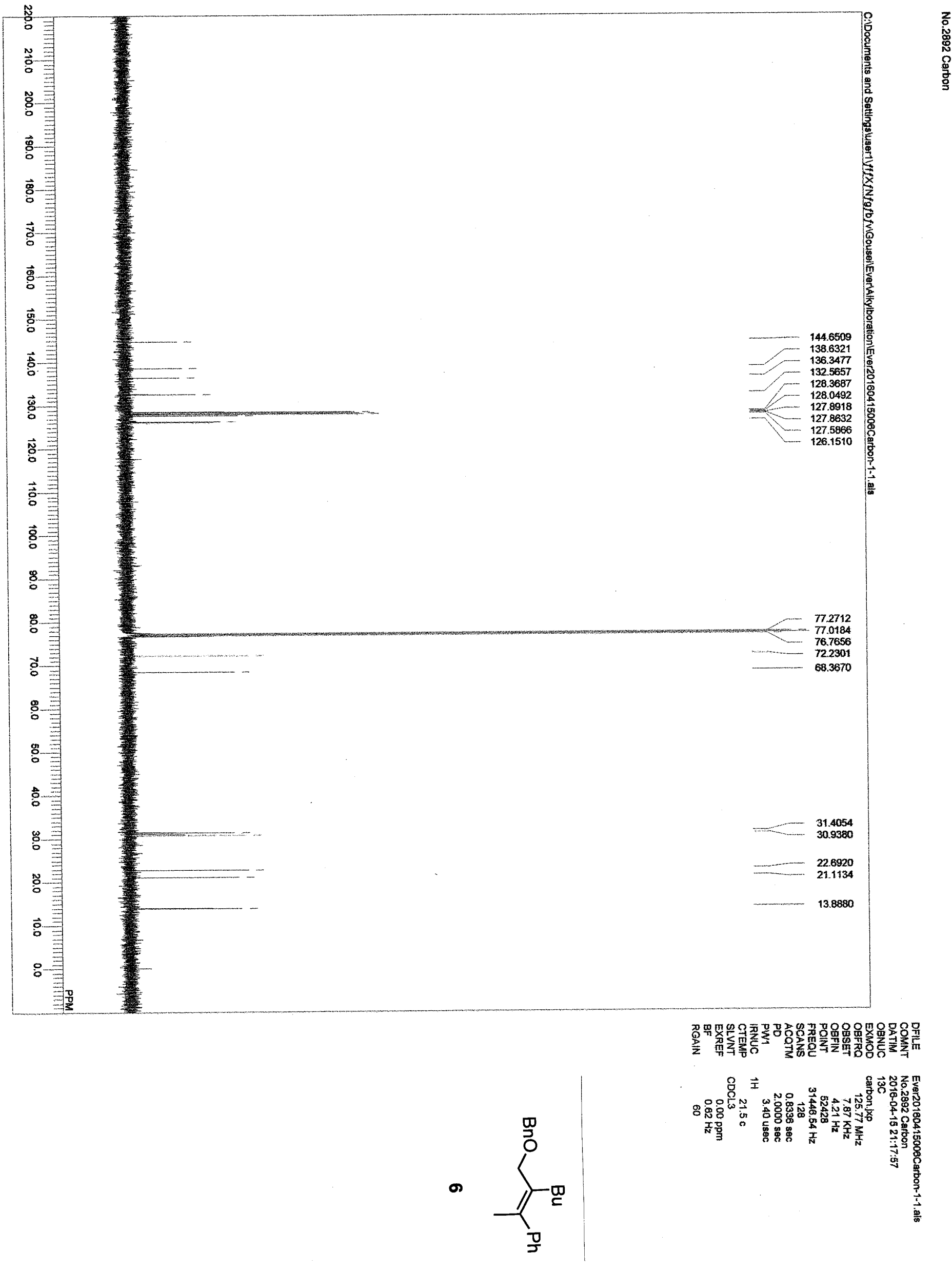




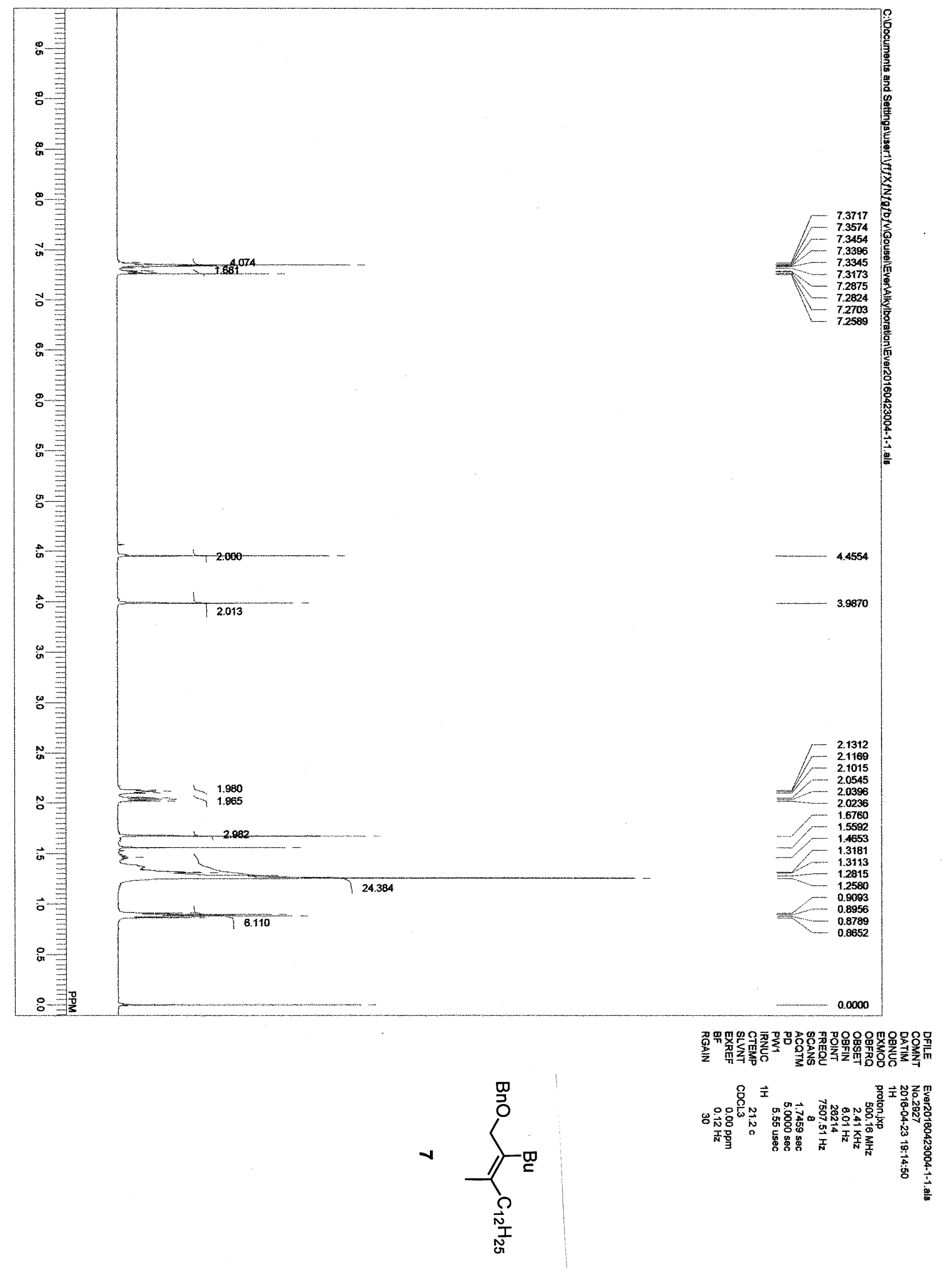




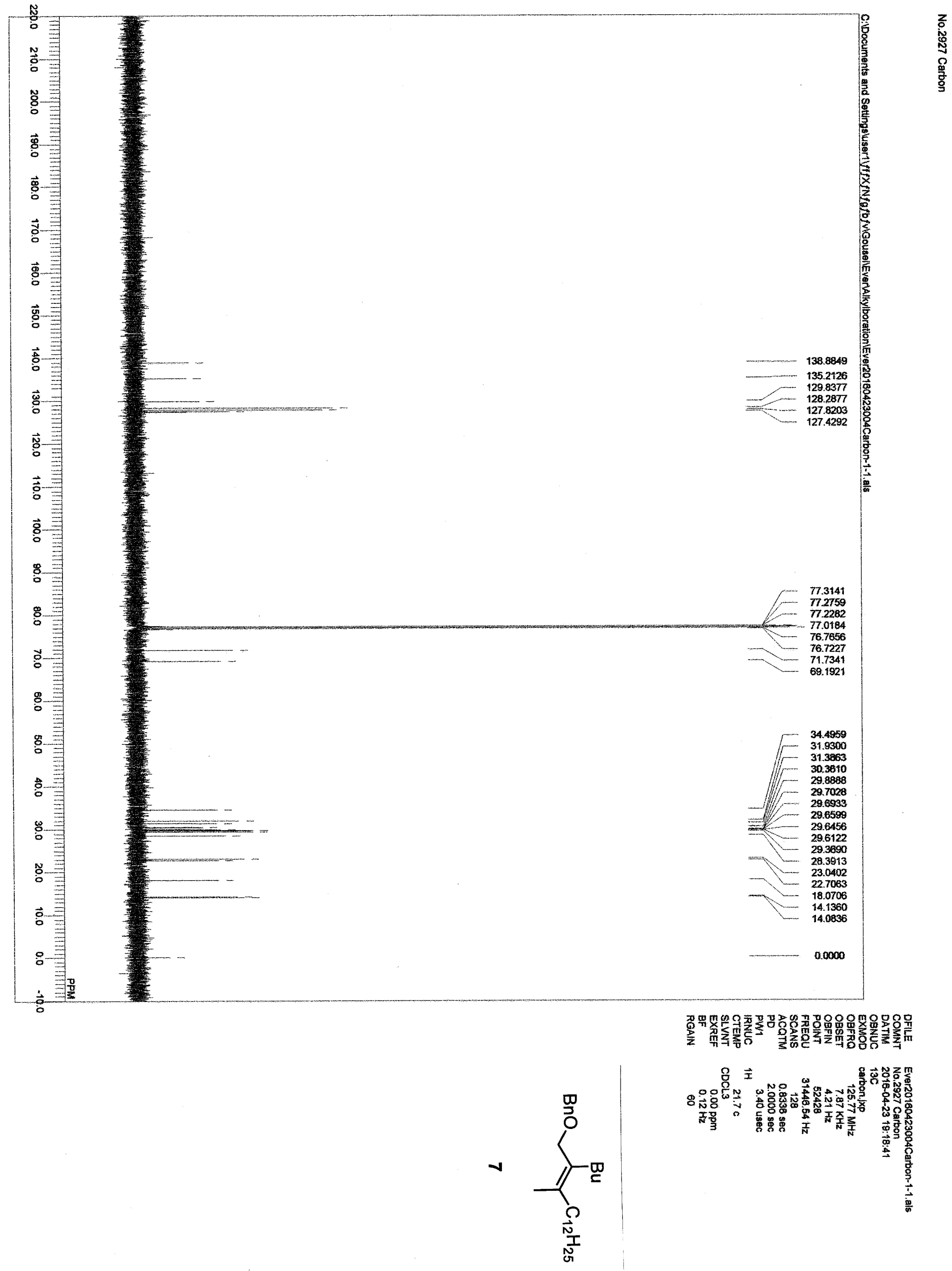

